\title{
A Global Formalism for Nonlinear Waves in Conservation Laws*
}

\author{
Eli L. Isaacson', Dan Marchesin'², C. Frederico Palmeira ${ }^{3}$, \\ and Bradley J. Plohr ${ }^{4}$
}

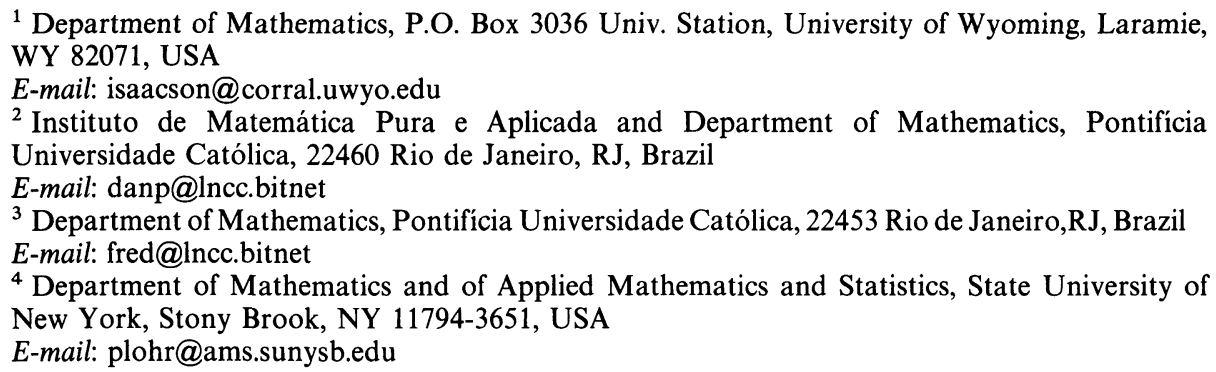

Received July 19, 1991; in revised form November 15, 1991

\begin{abstract}
We introduce a unifying framework for treating all of the fundamental waves occurring in general systems of $n$ conservation laws. Fundamental waves are represented as pairs of states statisfying the Rankine-Hugoniot conditions; after trivial solutions have been eliminated by means of a blow-up procedure, these pairs form an $(n+1)$-dimensional manifold $\mathscr{W}$, the fundamental wave manifold. There is a distinguished $n$-dimensional submanifold of $\mathscr{W}$ containing a single one-dimensonal foliation that represents the rarefaction curves for all families. Similarly, there is a foliation of $\mathscr{W}$ itself that represent shock curves. We identify other $n$-dimensional submanifolds of $\mathscr{W}$ that are naturally interpreted as boundaries of regions of admissible shock waves. These submanifolds also have one-dimensional foliations, which represent curves of composite waves. This geometric framework promises to simplify greatly the study of the stability and bifurcation properties
\end{abstract}

* This work was supported in part by: the NSF/CNPq U.S.-Latin America Cooperative Science Program under Grant INT-8612605; the Institute for Mathematics and its Applications with funds provided by the National Science Foundation; the Air Force Office of Scientific Research under Grant AFOSR 90-0075; the National Science Foundation under Grant 8901884; the U.S. Department of Energy under Grant DE-FG02-90ER25084; the U.S. Army Research Office under Grant DAAL03-89-K-0017; the Financiadora de Estudos e Projetos; the Conselho Nacional de Desenvolvimento Científico e Tecnológica $(\mathrm{CNPq})$; the Fundação de Amparo à Pesquisa do Estado do Rio de Janeiro (FAPERJ); the Coordenação de Aperfeiçamento de Pessoal de Ensino Superior (CAPES); and the Sociedade Brasileira de Matemática (SBM) 
of global solutions of Riemann problems for mixed hyperbolic-elliptic systems. In particular, bifurcations of wave curves can be understood as resulting from loss of transversality between foliations and admissibility boundaries.

\section{Introduction}

Realistic systems of conservation laws exhibit complicated interactions among nonlinear waves. This complexity presents a major obstacle to understanding solutions of Riemann initial-data problems. The present work introduces a global framework for describing nonlinear waves in general systems of conservation laws. This formalism provides a unified setting for all basic constructs used in solving Riemann problems - fundamental waves, wave curves, and the loci at which they bifurcate. These constructs correspond, respectively, to points, foliations, and submanifolds of a single manifold, which we call the fundamental wave manifold $\mathscr{W}$. When phrased in terms of $\mathscr{W}$, the description of solutions of Riemann problems and the study of their stability and bifurcation properties are considerably simplified.

A system of conservation laws models the time evolution of a continuum. When the motion of the continuum is one-dimensional, the system takes the form

$$
U_{t}+F(U)_{x}=0
$$

where the vector $U(x, t)$ represents the state of the continuum. Because the flux function $F$ depends nonlinearly on $U$, solutions of system (1.1) typically form discontinuities. For example, in gas dynamics, these discontinuities represent shock waves, as has long been known. Such nonlinear waves occur in shock-tube experiments, which were analyzed by Riemann [48] in 1858. These experiments have piecewise constant initial conditions; analogously, an initial-value problem for Eq. (1.1) with initial data

$$
U(x, t=0)= \begin{cases}U_{L} & \text { if } x<0 \\ U_{R} & \text { if } x>0\end{cases}
$$

is called a Riemann problem.

Gas dynamics also guided the elegant mathematical work of Lax [33] in 1957 on local Riemann problems, for which $U_{R}$ is close to $U_{L}$. In this setting, solutions are composed of small-amplitude nonlinear waves. Each wave corresponds to a mode of a linearization of system (1.1), and behaves essentially as the solution of the simplest nonlinear scalar conservation law, Burgers' equation. Because different modes propagate at distinct speeds, local Riemann problems can be solved mode by mode. The principal task is to construct wave curves, which are one-parameter families of solutions associated with a particular mode; solutions of Riemann problems are then obtained by successively traversing the wave curves for the several modes. Existence and uniqueness of solutions follows from the mutual transversality of wave curves, which reflects the weak mode coupling.

This classical approach to solving local Riemann problems has been extended by several authors $[45,58,60,9,35,36,37,10,38]$, who identify classes of conservation laws for which global Riemann problems can be solved. For instance, Oleĭnik [45] constructed solutions for an arbitrary scalar conservation law; and for the class of systems studied by Liu $[36,37]$, each mode can exhibit wave 
patterns as complicated as those for scalar equations. To guarantee existence and uniqueness of solutions, these authors assume that wave curves extend to infinity and are mutually transverse. Such hypotheses are rather restrictive, however; in essence, the coupling between different nonlinear modes must be weak.

While the classical construction works remarkably well even for large-amplitude waves in gas dynamics, it is not adequate for magnetohydrodynamics, elasticity, and multiphase flow. In these important applications, waves can interact strongly, so that wave curves violate the usual hypotheses on asymptotic behavior and transversality. Moreover, the nonlinear coupling causes modes to mix and lose their identifications with linearized modes; this leads to waves (transitional and overcompressive waves) that have no counterparts in the classical theory. Much has been learned about global Riemann problems by studying model systems using mathematical analysis and computer-aided exploration $[26,49,55,54,24,28,29,21,53,23,19$, $1,25]$. However, the classical approach has yielded solutions with conspicuously complicated descriptions, often requiring myriads of intricate diagrams. Standing in contrast to the simplicity and elegance of the local theory, such complexity poses an obstacle to understanding solutions of global Riemann problems.

In this paper we develop a new approach. Its basis is a geometric construction that represents all fundamental waves, both classical and nonclassical, as points in a single space. No special features need to be assumed of the conservation laws. Our thesis is that the strongly nonlinear phenomena associated with large-amplitude waves are reflected in the global properties of this spcae. This global perspective leads to a striking unification of ideas.

Central to our construction is the Rankine-Hugoniot condition, which embodies the physical conservation principle applied to a discontinuous solution. A jump discontinuity at each instant, is characterized by its speed of propagation $s$ and by the states $U_{-}$and $U_{+}$immediately to its left and right, respectively; the conservation laws require that these quantities satisfy the Rankine-Hugoniot condition

$$
-s\left[U_{+}-U_{-}\right]+F\left(U_{+}\right)-F\left(U_{-}\right)=0 .
$$

Triples $\left(U_{-}, U_{+}, s\right)$ satisfying Eq. (1.3) are of three types: (a) solutions with $U_{+} \neq U_{-}$, representing shock waves; (b) limits, as $\left|U_{+}-U_{-}\right| \rightarrow 0$, of shock wave solutions, which represent rarefaction waves; for these solutions, $s$ is in eigenvalue of the Jacobian $F^{\prime}(\bar{U})$ at $\bar{U}:=U_{+}=U_{-}$; and (c) solutions with $U_{+}=U_{-}$and other values of $s$, which represent constant states in solutions of Riemann problems.

The Rankine-Hugoniot condition represents $n$ equations for $2 n+1$ unknowns; however it is singular on the set of rarefaction solutions, so that it fails to define an $(n+1)$-dimensional manifold. We remove this singularity using a blow-up procedure. In essence, this procedure introduces a set of $n-1$ angles $\Omega$ to represent the orientation of the jump $U_{+}-U_{-}$even when $U_{+}=U_{-}$. Thus we write $U_{+}-U_{-}=\operatorname{Rr}(\Omega)$, where $R$ is a signed radial quantity and $r(\Omega)$ is the unit vector corresponding to $\Omega$. The midpoint $\bar{U}:=\frac{1}{2}\left(U_{-}+U_{+}\right)$serves as the remaining coordinate. The blow-up construction is intrinsic, i.e., independent of local coordinates. This is because the coordinates $(\bar{U}, R, \Omega, s)$ are needed only near the diagonal $U_{+}=U_{-}$; the coordinates $\left(U_{-}, U_{+}, s\right)$ work away from the diagonal. Thus our construction is invariant under nonlinear changes of variables in the conservation laws that preserve the jump conditions. 
Regarded as a relationship among the variables $\bar{U}, R, \Omega$, and $s$, the Rankine-Hugoniot equation (1.3) takes the form $R \cdot \mathscr{F}(\bar{U}, R, \Omega, s)=0$. Thus the solution set is the union of two subsets. The first is the trivial subset where $R=0$. The second is the subset $\mathscr{W}$ where $\mathscr{F}=0$, an element of which represents a shock wave when $R \neq 0$ and a point on a rarefaction fan when $R=0$. Since rarefaction points also belong to the trivial subset, the two subsets intersect, causing a singularity. Therefore we dispense with the trivial solutions $R=0$ and focus on $\mathscr{W}$.

In Sect. 2 we establish that $\mathscr{W}$ is a smooth manifold of dimension $n+1$, the closure of the set of shock points. We therefore call $\mathscr{W}$ the fundamental wave manifold. To obtain this result we adopt a mild hypothesis that holds for generic systems of conservation laws; in particular, systems of mixed hyperbolic-elliptic type are allowed. While we make this hypothesis to simplify the exposition, the concepts we develop are useful for completely general systems, as we show by example.

A significant feature of the global approach is that both rarefaction and shock waves are represented within the single manifold $\mathscr{W}$. Thus the wave manifold implements the heuristic idea that shock waves with infinitesimal strength are infinitesimal rarefaction fans. In Sect. 3 we show that rarefaction points form an $n$-dimensional submanifold $\mathscr{C}$ of $\mathscr{W}$, called the characteristic manifold. At a point of $\mathscr{C}, r(\Omega)$ is an eigenvector of $F^{\prime}(\bar{U})$ with eigenvalue (i.e., characteristic speed) $s$. Information about the behavior of eigenvalues is encoded in the natural projection that maps $\mathscr{C}$ to state space. When the system is strictly hyperbolic, $\mathscr{C}$ is an $n$-sheeted covering manifold for state space; but for general systems, the projection has singularities precisely when $F^{\prime}(\bar{U})$ has multiple eigenvalues, i.e., coincident wave speeds. Geometrically, the sheets of $\mathscr{C}$ are glued together along this coincidence set $\mathscr{E}$, and $\mathscr{C}$ need not cover all of state space, the gaps being regions where the system is elliptic.

As described in Sect. 4 , the $n$ families of rarefaction curves for the system of conservation laws are projections of a single family of curves in $\mathscr{C}$. These curves form a one-dimensional foliation that is naturally induced by the geometry of $\mathscr{C}$. Most singularities of rarefaction curves are eliminated by lifting them to $\mathscr{C}$; for instance, cusps are artifacts of the projection. Singularities in the foliation of $\mathscr{C}$ can occur, however, at certain points in $\mathscr{E}$. These singularities are essential because they determine the global geometry of rarefaction curves. Also important is how the wave speed $s$ varies along rarefaction curves, the topic of Sect. 5. The set of stationary points for $s$, where genuine nonlinearity fails, is the projection of a subset $\mathscr{I}$ of $\mathscr{C}$ called the inflection locus. The wave speed attains an extremum at a point of $\mathscr{I}$ provided that the rarefaction curve is transverse to $\mathscr{I}$ there; the set $\mathscr{H}_{0}$ where this fails plays a role in wave curve bifurcation.

As discussed in Sect. 6, the wave manifold itself is foliated by two families of curves, the level curves where either $U_{-}$or $U_{+}$is fixed. These curves are called shock curves because they project onto the classical shock curves in state space. Whereas the classical shock curves display multiple branches near the fixed state, shock curves in $\mathscr{W}$ do not: the blow-up procedure eliminates this primary bifurcation. Secondary bifurcations occur where one of the shock foliations is singular; thus the secondary bifurcation loci $\mathscr{B}_{R}$ and $\mathscr{B}_{L}$ are important for the global geometry of shock curves. As is natural, rarefaction singularities occur where $\mathscr{B}_{R}$ and $\mathscr{B}_{L}$ intersect $\mathscr{C}$.

In Sect. 7 we study the behavior of the shock speed $s$ along shock curves. Stationary points for $s$ occur on $n$-dimensional submanifolds, called the sonic 
manifolds $\mathscr{S}_{L}$ and $\mathscr{S}_{R}$, where $s$ is an eigenvalue of $F^{\prime}\left(U_{-}\right)$or $F^{\prime}\left(U_{+}\right)$, respectively. This result is a geometric version of the Bethe-Wendroff theorem. The shock speed attains an extremum at a sonic point if the shock curve is transverse there; loss of transversality occurs on the hysteresis loci $\mathscr{H}_{L}$ and $\mathscr{H}_{R}$, and this causes wave curve bifurcation. The intersection of the sonic loci $\mathscr{S}_{L}$ and $\mathscr{S}_{R}$ is the union of the inflection locus $\mathscr{I}$ and the locus $\mathscr{D}$ comprising doubly sonic shock waves, another wave curve bifurcation locus.

As we show in Sect. 8, the sonic manifolds themselves are foliated by families of curves that are induced naturally by the shock and rarefaction foliations. These curves parameterize solutions of the conservation laws called composite waves, each comprising a sonic shock wave adjacent to a rarefaction wave. The composite foliation also possesses singularities that affect its global structure.

Throughout Sects. $2-8$ we systematically illustrate the theory by applying it to several representative examples of conservation laws: gas dynamics, models with quadratic polynomial flux functions, and a class of models studied by KeyfitzKranzer and Isaacson-Temple.

In Sect. 9 we discuss how the wave manifold framework can shed light on two fundamental problems in the theory of conservation laws: the physical admissibility of shock waves, which is determined by properties of dynamical systems that are parameterized by the points of $\mathscr{W}$; and the bifurcation of wave curves, which can be characterized as loss of transversality between the rarefaction, shock, and composite foliations and the boundary of the region of admissible waves. Another application of the global approach is treated in a companion paper [42] that concerns systems of two conservation laws with flux functions that are close to a quadratic polynomial; it is proved that the fundamental wave manifold and its shock foliations are stable under perturbations of the flux function.

Throughout the main body of the paper we assume, for simplicity, that the state space is $\mathbf{R}^{n}$. In the Appendix we provide an intrinsic definition of the fundamental wave manifold when the state space is a general smooth manifold. To do so we extend the classical blow-up construction.

An enlightening discussion of the role of the fundamental wave manifold in a broader context is found in the review article of Glimm [16]. Conversations between this reviewer and the present authors were instrumental to the inception of this paper.

\section{The Fundamental Wave Manifold $\mathscr{W}$}

The simplifying feature of Riemann problems is invariance under the scalings $(x, t) \mapsto(\alpha x, \alpha t)$ for all $\alpha>0$. Therefore we seek scale-invariant solutions. For instance, the discontinuous scale-invariant function

$$
U(x, t)= \begin{cases}U_{-} & \text {if } x<s t, \\ U_{+} & \text {if } x>s t,\end{cases}
$$

is a solution when $U_{-}, U_{+}$, and $s$ satisfy the Rankine-Hugoniot jump condition

$$
-s\left[U_{+}-U_{-}\right]+F\left(U_{+}\right)-F\left(U_{-}\right)=0,
$$

which embodies the physical conservation principle applied to a jump discontinuity. The solution (2.1) represents a shock wave propagating at constant speed $s$. On 
the other hand, a differentiable scale-invariant function $U(x, t)=\tilde{U}(x / t)$ is a solution for $t>0$ if and only if

$$
\left[-s+F^{\prime}(\tilde{U})\right] \tilde{U}^{\prime}=0 \quad \text { with } \quad s=x / t
$$

it corresponds physically to a rarefaction wave. Equation (2.3) can be viewed as arising from the Rankine-Hugoniot condition in the limit of weak shock waves $\left(\left|U_{+}-U_{-}\right| \rightarrow 0\right)$. The general scale-invariant solution of a Riemann problem can be decomposed into wave groups, which are bounded by sectors where the solution is constant. Each wave group is composed of adjoining rarefaction and shock waves.

In this section we construct an $(n+1)$-dimensional manifold that parameterizes solutions of Eq. (2.2). Triples $\left(U_{-}, U_{+}, s\right)$ satisfying Eq. (2.2) are of three types: (a) solutions with $U_{+} \neq U_{-}$, representing shock waves; (b) limits, as $\left|U_{+}-U_{-}\right| \rightarrow 0$, of shock wave solutions, which represent rarefaction waves; for these solutions, $s$ is an eigenvalue of the Jacobian $F^{\prime}(\bar{U})$ at $\bar{U}:=U_{+}=U_{-}$; and (c) solutions with $U_{+}=U_{-}$and other values of $s$, which represent constant states in solutions of Riemann problems.

We assume for simplicity that $U(x, t)$ belongs to the state space $\mathscr{U}:=\mathbf{R}^{n}$ and that the flux $F$ is a smooth map from $\mathscr{U}$ to $\mathbf{R}^{n}$. (An example where $\mathscr{U}$ is a convex open subset of $\mathbf{R}^{n}$ is studied below; the case in which $\mathscr{U}$ is a more general $n$-dimensional manifold is treated in the Appendix.) The solution set for Eq. (2.2) is a subset of the ambient space $\mathscr{P}:=\mathscr{U} \times \mathscr{U} \times \mathbf{R}$, namely the zero-set of the function $\mathscr{H}: \mathscr{P} \rightarrow \mathbf{R}^{n}$ defined by

$$
\mathscr{H}\left(U_{-}, U_{+}, s\right):=-s\left[U_{+}-U_{-}\right]+F\left(U_{+}\right)-F\left(U_{-}\right) .
$$

It is a submanifold of $\mathscr{P}$ near any point at which the derivative of $\mathscr{H}$ has full rank $n$. This derivative is

$$
d \mathscr{H}\left(U_{-}, U_{+}, s\right)=-\left[U_{+}-U_{-}\right] d s+\left[-s+F^{\prime}\left(U_{+}\right)\right] d U_{+}-\left[-s+F^{\prime}\left(U_{-}\right)\right] d U_{-} .
$$

At points $U_{+} \neq U_{-}$representing shock waves. $d \mathscr{H}$ has full rank except in rather pathological circumstances. Likewise, $d \mathscr{H}$ has full rank at points representing constant states. At rarefaction points, however, $U_{+}=U_{-}$and $s$ is an eigenvalue of $F^{\prime}\left(U_{+}\right)=F^{\prime}\left(U_{-}\right)$, so that $d \mathscr{H}$ has rank less than $n$. This singularity occurs where the closure of the set of constant solutions intersects the set of rarefaction solutions. To remove it, we employ the technique of blowing up. (See the Appendix for a general discussion of this construction.)

The blowing up process resolves the singularity at $U_{+}=U_{-}$using a variable $\Omega$ to represent the orientation of the vector $\Delta U:=U_{+}-U_{-}$even when $\Delta U=0$. Specifically, we introduce $R \in \mathbf{R}$ and $\Omega \in S^{n-1}$ to serve as polar coordinates for $\Delta U=\operatorname{Rr}(\Omega)$; here $S^{n-1}$ is the $(n-1)$-dimensional sphere, and the coordinate $\Omega$ parameterizes unit vectors $r(\Omega)$ in the tangent space of $\mathscr{U}$ at the midpoint $\bar{U}:=\frac{1}{2}\left(U_{-}+U_{+}\right)$. Notice that $(R, \Omega)$ is not uniquely determined from $\Delta U$ : the antipodal point $(-R,-\Omega$ ) works as well $(-\Omega$ being antipodal to $\Omega$ on the sphere); and $\Omega$ is arbitrary when $\Delta U=0$. The blowing up technique exploits the latter ambiguity. In fact, singularities at $\Delta U=0$ are less severe in terms of $(R, \Omega)$ coordinates. 
Consider the space $\hat{\mathscr{P}}:=\mathscr{U} \times \mathbf{R} \times S^{n-1} \times \mathbf{R}$. We associate to each point $(\bar{U}, R, \Omega, s) \in \hat{\mathscr{P}}$ the left and right states

$$
U_{-}:=\bar{U}-\frac{1}{2} \operatorname{Rr}(\Omega), \quad U_{+}:=\bar{U}+\frac{1}{2} \operatorname{Rr}(\Omega),
$$

respectively. Thus $\bar{U}=\frac{1}{2}\left(U_{-}+U_{+}\right)$is the midpoint between $U_{+}$and $U_{-}$, while $R$ and $\Omega$ determine the difference $\operatorname{Rr}(\Omega)=U_{+}-U_{-}$. Notice that $U_{-}$and $U_{+}$are each unchanged when $(\bar{U}, R, \Omega, s)$ is replaced by the antipodal point $(\bar{U},-R,-\Omega, s)$. We therefore define $\mathscr{P}^{*}$ to be the space obtained from $\hat{\mathscr{P}}$ by identifying these points, and let $\sigma: \hat{\mathscr{P}} \rightarrow \mathscr{P} *$ denote the identification map. Then $\mathscr{P} *$ is a smooth manifold and $\hat{\mathscr{P}}$ is a two-fold covering manifold for $\mathscr{P}^{*}[32$, p. 44]. Indeed: the quotient topology on $\mathscr{P}^{*}$ is Hausdorff; and each point $(\bar{U}, R, \Omega, s)$ in $\hat{\mathscr{P}}$ has a neighborhood $\mathcal{O}$ disjoint from its image $-\mathcal{O}$ under the antipodal transformation, so that $\sigma$ defines a homeomorphism between $\mathcal{O}$ and $\sigma[\mathcal{O}]$. Thus $\bar{U}, R, \Omega$, and $s$ serve as local coordinates for $\mathscr{P} *$ on $\sigma[\mathcal{O}]$.

The map from $\hat{\mathscr{P}}$ to $\mathscr{P}$ defined by Eqs. (2.6) induces a map $\pi: \mathscr{P}^{*} \rightarrow \mathscr{P}$ because antipodal points are carried to the same image. The induced map $\pi$ is a diffeomorphism of the subset of $\mathscr{P} *$ where $R \neq 0$ with the subset of $\mathscr{P}$ where $U_{+} \neq U_{-}$, but each point on the diagonal $\Delta=\left\{\left(U_{-}, U_{+}, s\right) \in \mathscr{P}: U_{+}=U_{-}\right\}$is the image under $\pi$ of a projective space $\mathbf{R} P^{n-1}$ embedded in $\mathscr{P} *$. Geometrically, if $\Omega$ belongs to the $\mathbf{R} P^{n-1}$ over $(\bar{U}, \bar{U}, s) \in \mathscr{P}$, then $r(\Omega)$ spans a line through the origin of the tangent space of $\mathscr{U}$ at $\bar{U}$. (See the Appendix.) The hypersurface $R=0$ in $\mathscr{P} *$ is denoted $\mathscr{P}_{0}^{*}:=\mathscr{U} \times\{0\} \times \mathbf{R} P^{n-1} \times \mathbf{R}$.

Definition 2.1. The $(2 n+1)$-dimensional manifold $\mathscr{P}^{*}$ is called the blow-up of $\mathscr{P}=\mathscr{U} \times \mathscr{U} \times \mathbf{R}$ over its diagonal $\Delta$. The collapsing map is $\pi: \mathscr{P}^{*} \rightarrow \mathscr{P}$. The $2 n$-dimensional manifold $\mathscr{P}_{0}^{*}$, which is the preimage under $\pi$ of the diagonal of $\mathscr{P}$, is called the exceptional hypersurface.

The relationship between these spaces is summarized in the following diagram:

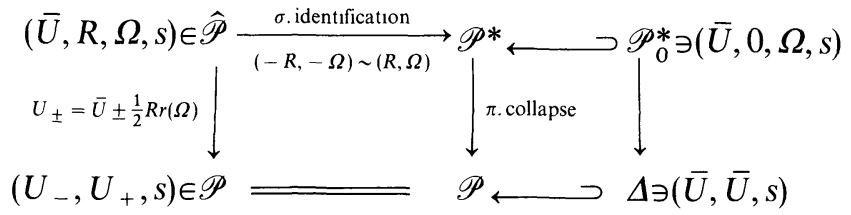

Example. When $n=2$, we take $\Omega \in[0,2 \pi)$ to be the polar angle, so that $r(\Omega)=$ $(\cos \Omega, \sin \Omega)$. The antipodal map carries $(\bar{U}, R, \Omega, s)$ to $(\bar{U},-R, \Omega+\pi, s)$. To serve as coordinates for $\mathscr{P}^{*},(R, \Omega)$ can be restricted to the range $\mathbf{R} \times[0, \pi]$, with each pair $(-R, \pi)$ identified with $(R, 0)$. Therefore $\mathscr{P}^{*}=\mathscr{U} \times M_{2} \times \mathbf{R}$, where $M_{2}$ is a Möbius strip. For each fixed $\bar{U}$ and $s, \mathscr{P}^{*}$ can be viewed as the ruled surface $\Delta u=R \cos \Omega$ and $\Delta v=R \sin \Omega$ embedded in $(\Delta u, \Delta v, \Omega)$-space, as depicted in Fig. 2.1. The collapsing map corresponds to vertical projection.

Remark. The blow-up construction is still feasible, although somewhat more complicated, when the state space $\mathscr{U}$ is a general $n$-dimensional manifold, as discussed in the Appendix. The principal difference is that the coordinates $(\bar{U}, R, \Omega, s)$ can be used only in small neighborhoods of points on the exceptional hypersurface. Even though $\mathscr{U}$ is assumed to be $\mathbf{R}^{n}$ in the main body of the paper, 


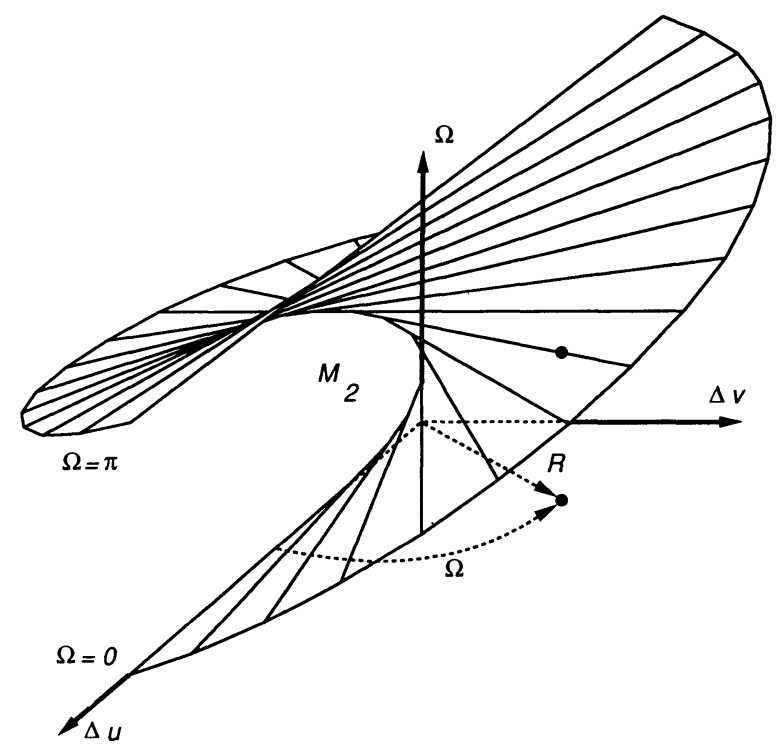

Fig. 2.1. The slice $M_{2}$ of $\mathscr{P} *$ with fixed $\bar{U}$ and $s$, as embedded in the space with coordinates $(\Delta u, \Delta v, \Omega)$, in the case $n=2$. Above each point $\Delta U \neq 0$ lies a unique point of $M_{2}$, but the vertical projection is singular along the $\Omega$-axis

all results carry over to the general case provided that we employ the coordinates $\left(U_{-}, U_{+}, s\right)$ away from $\mathscr{P}_{0}^{*}$.

The subset of $\mathscr{P}$ defined by Eq. (2.2) is singular along the diagonal $U_{+}=U_{-}$; however, it is the projection of a less singular subset of the blow-up $\mathscr{P}^{*}$, which we now construct. To this end, notice that there exists a matrix-valued function $A$ that satisfies

$$
F\left(U_{+}\right)-F\left(U_{-}\right)=R \cdot A(\bar{U}, R, \Omega) r(\Omega)
$$

for instance,

$$
A(\bar{U}, R, \Omega):=\int_{-1 / 2}^{1 / 2} F^{\prime}(\bar{U}+\alpha R r(\Omega)) d \alpha
$$

satisfies Eq. (2.7). We regard $A$ as defined on both $\hat{\mathscr{P}}$ and $\mathscr{P}^{*}$. In terms of the composite map $\mathscr{H} \circ \pi: \mathscr{P P}^{*} \rightarrow \mathbf{R}^{n}$, the Rankine-Hugoniot condition becomes

$$
(\mathscr{H} \circ \pi)(\bar{U}, R, \Omega, s):=R \cdot[-s+A(\bar{U}, R, \Omega)] r(\Omega)=0 .
$$

Examples.

1. A prototype for the equations governing gas flow is the system of two conservation laws $u_{t}+p(v)_{x}=0$ and $v_{t}-u_{x}=0$. In terms of $R \in \mathbf{R}$ and $\Omega \in[0, \pi], u_{ \pm}=$ $\bar{u} \pm \frac{1}{2} R \cos \Omega$ and $v_{ \pm}=\bar{v} \pm \frac{1}{2} R \sin \Omega$. Let

$$
\Psi\left(v_{-}, v_{+}\right):=-\int_{0}^{1} p^{\prime}\left((1-\alpha) v_{-}+\alpha v_{+}\right) d \alpha
$$

denote the average of $-p^{\prime}$ over the interval $\left[v_{-}, v_{+}\right]$. Then the Rankine-Hugoniot 
condition requires that either (a) $u_{+}=u_{-}$and $v_{+}=v_{-}$or (b) $s^{2}=\Psi\left(v_{-}, v_{+}\right)$, $\cot \Omega=-s$, and $u_{ \pm}=\bar{u} \mp \frac{1}{2} s\left(v_{+}-v_{-}\right)$.

2. A quadratic model is a system of conservation laws for which the flux $F$ depends quadratically on $U$. For such models, Eq. (2.8) shows that $A(\bar{U}, R, \Omega)=F^{\prime}(\bar{U})$. According to Eq. (2.9), $\left(U_{-}, U_{+}, s\right)$ satisfies the Rankine-Hugoniot condition if and only if either (a) $U_{+}=U_{-}$or (b) the difference $U_{+}-U_{-}=\operatorname{Rr}(\Omega)$ is an eigenvector of $F^{\prime}(\bar{U})$, the Jacobian evaluated at the midpoint $\bar{U}=\frac{1}{2}\left(U_{-}+U_{+}\right)$, with the shock speed $s$ being the eigenvalue. (This is known as the midpoint rule [24].)

3. The models studied by Keyfitz and Kranzer [31] and by Isaacson and Temple $[22,27]$ have fluxes of the form $F(U)=\Phi(U) U$, where $\Phi: \mathscr{U} \rightarrow \mathbf{R}$. We will refer to them as KKIT models. Let

$$
\begin{aligned}
& \bar{\Phi}(\bar{U}, R, \Omega):=\frac{1}{2}\left[\Phi\left(U_{-}\right)+\Phi\left(U_{+}\right)\right], \\
& \Psi(\bar{U}, R, \Omega):=\int_{-1 / 2}^{1 / 2} \Phi^{\prime}(\bar{U}+\alpha R r(\Omega)) d \alpha .
\end{aligned}
$$

Then $A(\bar{U}, R, \Omega):=\bar{\Phi}(\bar{U}, R, \Omega)+\bar{U} \Psi(\bar{U}, R, \Omega)$ satisfies Eq. (2.7). (In this example, it is better not to adopt definition (2.8).) As a result, Eq. (2.9) holds if and only if either (a) $R=0$, (b) $\Psi(\bar{U}, R, \Omega) r(\Omega)=0$ and $s=\bar{\Phi}(\bar{U}, R, \Omega)$, or (c) $\bar{U}=\bar{\kappa} r(\Omega)$ for some $\bar{\kappa} \in \mathbf{R}$ and $s=\bar{\Phi}(\bar{U}, R, \Omega)+\Psi(\bar{U}, R, \Omega) \bar{U}$. In case (b), a solution corresponds to a contact discontinuity with $\Phi\left(U_{+}\right)=s=\Phi\left(U_{-}\right)$. For a solution in case (c), $U_{-}, U_{+}$, and $\bar{U}$ all lie along the line generated by $r(\Omega): U_{ \pm}=\kappa_{ \pm} r(\Omega)$ with $\kappa_{ \pm}:=\bar{\kappa} \pm \frac{1}{2} R$. If the scalar flux function $g_{\Omega}$ is defined by $g_{\Omega}(\kappa):=\kappa \Phi(\kappa r(\Omega))$, then the solution corresponds to a shock wave for the scalar conservation law $\kappa_{t}+g_{\Omega}(\kappa)_{x}=0$, since $s=\int_{-1 / 2}^{1 / 2} g_{\Omega}^{\prime}(\bar{\kappa}+\alpha R) d \alpha=\int_{0}^{1} g_{\Omega}^{\prime}\left((1-\alpha) \kappa_{-}+\alpha \kappa_{+}\right) d \alpha$ is the slope of the chord on the graph of $g_{\Omega}$.

Equation (2.9) explicitly displays the trivial solution $R=0$ that is to be eliminated by blowing up. Although dividing $\mathscr{H} \circ \pi$ by $R$ does not yield a well-defined map on $\mathscr{P}^{*}$, it does define a map $\mathscr{F}: \hat{\mathscr{P}} \rightarrow \mathbf{R}^{n}$ on the covering space $\hat{\mathscr{P}}$ :

$$
\mathscr{F}(\bar{U}, R, \Omega, s):=[-s+A(\bar{U}, R, \Omega)] r(\Omega) .
$$

Definition 2.2. Let $\hat{\mathscr{W}} \subseteq \hat{\mathscr{P}}$ denote the zero-set of $\mathscr{F}: \hat{\mathscr{P}} \rightarrow \mathbf{R}^{n}$. The fundamental wave set $\mathscr{W} \subseteq \mathscr{P} *$ is the image of $\hat{\mathscr{W}}$ under the identification map $\sigma: \hat{\mathscr{P}} \rightarrow \mathscr{P} *$.

The fundamental wave set generalizes the manifold constructed by Marchesin and Palmeira [42] for a class of systems of two conservation laws with quadratic fluxes. (The construction of ref. [42] is based on the ambient space $\mathscr{U} \times \mathscr{U}$ rather than $\mathscr{P}=\mathscr{U} \times \mathscr{U} \times \mathbf{R}$. This is equivalent to the present approach because the shock speed $s$ can be recovered from $U_{-}$and $U_{+}$. See the Appendix for further discussion.)

\section{Examples.}

1. In gas dynamics, $u_{ \pm}$and $\Omega$ are explicit functions of $v_{-}, v_{+}, s$, and $\bar{u}$. Thus it is convenient to regard $\mathscr{W}$ as the set of points $\left(v_{-}, v_{+}, s, \bar{u}\right)$ such that $s^{2}=\Psi\left(v_{-}, v_{+}\right)$. Thus $\mathscr{W}=\mathscr{W}_{\text {base }} \times \mathbf{R}$, where the factor $\mathbf{R}$ corresponds to the velocity coordinate $\bar{u}$. To understand $\mathscr{W}$, are study the fixed-velocity surface $\mathscr{W}_{\text {base }}$.

For concreteness, take $p$ to be an isotherm for the van der Waals equation of state (see, e.g., refs. [57,52]): $\left(p+3 / v^{2}\right)(v-1 / 3)=8 T / 3$ for $v>1 / 3$. (Although this 


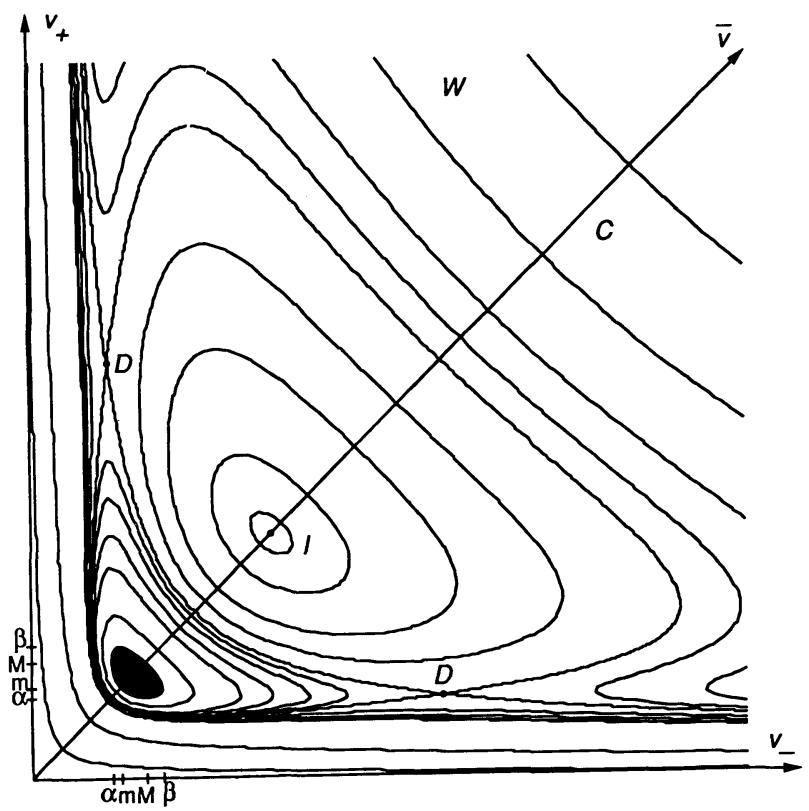

Fig.2.2. Level curves of $s^{2}=\Psi\left(v_{-}, v_{+}\right)$for a van der Walls gas. The black hole is the region $\mathscr{R}$ where $\Psi$ is negative. The speed $s$ tends to infinity at the two axes $v_{-}=0$ and $v_{+}=0$, and tends to zero along any other radial direction. Several features are marked for comparison with Fig. 7.2

equation of state illustrates nicely how elliptic behavior is accommodated in a global setting, we do not advocate its use as a physical model.) If $T>1, p^{\prime}$ is strictly negative everywhere, so that the system is strictly hyperbolic. In this case, $\mathscr{W}_{\text {base }}$ consists of two disconnected sheets, corresponding to the solutions $s=\Psi\left(v_{-}, v_{+}\right)^{1 / 2}$ and $s=-\Psi\left(v_{-}, v_{+}\right)^{1 / 2}$. Otherwise, if $T<1$, there is a hole $\mathscr{R}$ in the $\left(v_{-}, v_{+}\right)$-plane where $\Psi\left(v_{-}, v_{+}\right)<0$. Again there are two sheets, now lying over the complement of $\mathscr{R}$, which glue along the boundary of $\mathscr{R}$ (where $s=0$ ) to form a tube. The speed $s$ is needed as a coordinate for $\mathscr{W}_{\text {base }}$ in the neck of the tube. Level curves of $s^{2}$ are shown in Fig. 2.2.

2. For the quadratic models studied by Marchesin and Palmeira [42], $\mathscr{W}$ is a threedimensional manifold with the global topological structure of $M_{2} \times \mathbf{R}$, where $M_{2}$ is a Möbius strip.

3. For the KKIT models, $\mathscr{W}$ is the union of two manifolds: the contact manifold $\mathscr{W}_{\text {contact }}$ on which $\Psi(\bar{U}, R, \Omega) r(\Omega)=0$ and $s=\bar{\Phi}(\bar{U}, R, \Omega)$; and the ruled surface $\mathscr{W}_{\text {ruled }}^{\text {contact }}$ where $\bar{U}=\bar{\kappa} r(\Omega)$ for $\bar{\kappa} \in \mathbf{R}$ and $s=\bar{\Phi}(\bar{U}, R, \Omega)+\Psi(\bar{U}, R, \Omega) \bar{U}$. Although $\mathscr{W}_{\text {ruled }}$ has dimension $n+1, \mathscr{W}_{\text {contact }}$ has dimension $2 n-1$, and these two manifolds intersect. Thus $\mathscr{W}$ is not a manifold.

Theorem 2.3. Assume that zero is a regular value for $\mathscr{F}$, i.e., the derivative of $\mathscr{F}$ has rank $n$ at each point of $\hat{\mathscr{W}}$. Then the fundamental wave set $\mathscr{W}$ is an $(n+1)$-dimensional submanifold of the blow-up $\mathscr{P} *$ of $\mathscr{P}$ over its diagonal. Furthermore, $\hat{\mathscr{W}} \subseteq \widehat{\mathscr{P}}$ is a twofold covering manifold for $\mathscr{W}$. 
Proof. Since the derivative of $\mathscr{F}$ has rank $n$ at each point of $\hat{\mathscr{W}}, \hat{\mathscr{W}}$ is an $(n+1)-$ dimensional submanifold of $\hat{\mathscr{P}}$. Also, $\hat{\mathscr{W}}$ is invariant under the antipodal transformation because $\mathscr{F}(\vec{U},-R,-\Omega, s)=-\mathscr{F}(\bar{U}, R, \Omega, s)$. If $\mathcal{O} \subset \widehat{\mathscr{P}}$ is an open set disjoint from its antipodal image $-\mathcal{O}$, then the identification map $\sigma: \hat{P} \rightarrow \mathscr{P}^{*}$ establishes a diffeomorphism between $\hat{\mathscr{W}} \cap \mathcal{O}$ and $\mathscr{W} \cap \sigma[\mathcal{O}]$. Thus $\sigma$ restricts to a two-fold covering map from $\widehat{\mathscr{W}}$ onto $\mathscr{W}$.

The assumption that zero is a regular value for $\mathscr{F}$ is expected to hold for flux functions that are generic (in a suitable sense). Nevertheless there are important systems of conservation laws for which $\mathscr{W}$ is not a manifold; examples are discussed throughout the paper. To simplify the exposition, however, the following implicit assumption will be made in all theorems in the remainder of the paper.

Assumption 2.4. Zero is a regular value for $\mathscr{F}$.

In our analysis of $\mathscr{W}$, we will repeatedly invoke the following simple lemma.

Lemma 2.5. The function $\mathscr{F}$ is an even function of $R$. In particular, any partial derivative of $\mathscr{F}$ that has odd order with respect to $R$ vanishes at $R=0$.

Proof. The definition (2.7) of $A$ shows that $A(\bar{U},-R, \Omega)=A(\bar{U}, R, \Omega)$.

Intuitively, $\mathscr{W}$ contains shock and rarefaction points, but excludes constant states; see Fig. 2.3. In fact, it is the closure of shock wave solutions.

Proposition 2.6. The fundamental wave manifold $\mathscr{W}$ is the closure in $\mathscr{P} *$ of points $\left(U_{-}, U_{+}, s\right)$ with $U_{+} \neq U_{-}$satisfying $E q$. (2.2).

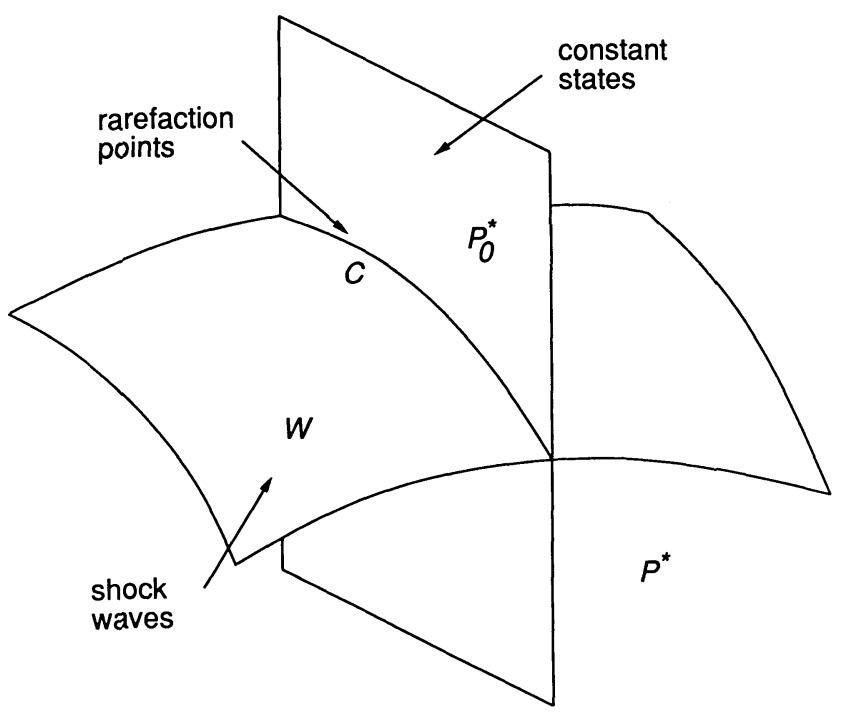

Fig. 2.3. A schematic picture of the $(2 n+1)$-dimensional space $\mathscr{P}^{*}$, the $2 n$-dimensional exceptional hypersurface $\mathscr{P}_{0}^{*}$, and the $(n+1)$-dimensional fundamental wave manifold $\mathscr{W}$. The wave manifold parameterizes shock wave solutions, while the exceptional hypersurface corresponds to constant states. The intersection of these manifolds is the $n$-dimensional characteristic manifold $\mathscr{C}$ that contains rarefaction points 
Prodf. We must show that $\mathscr{W}$ is the closure of points $(\bar{U}, R, \Omega, s) \in \mathscr{P} *$ with $R \neq 0$ such that $(\mathscr{H} \circ \pi)(\bar{U}, R, \Omega, s)=0$, i.e., $\mathscr{W}$ is the closure in $\mathscr{P}^{*}$ of $\mathscr{W} \backslash \mathscr{P}_{0}^{*}$. This is a consequence of the following lemma.

Lemma 2.7. The exceptional hypersurface $\mathscr{P}_{0}^{*} \subset \mathscr{P}^{*}$ is transverse to the submanifold $\mathscr{W}$.

Proof. The hypersurface $\hat{\mathscr{P}}_{0} \subset \widehat{\mathscr{P}}$ where $R=0$ is invariant under the antipodal transformation, so that it is a two-fold cover of $\mathscr{P}_{0}^{*} \subset \mathscr{P}^{*}$. Therefore it suffices to show that $\hat{\mathscr{P}}_{0}$ is transverse to $\hat{\mathscr{W}}$. But because $\mathscr{F}_{R}=0$ on $\hat{\mathscr{P}}_{0}$ by Lemma $2.5, d R$ is linearly independent of $d \mathscr{F}$ on $\dot{\hat{\mathscr{W}}} \cap \hat{\mathscr{P}}_{0}$.

Remark. Since the blow-up process is rather involved, one might question whether blowing up $\mathscr{P}$ is essential for defining $\mathscr{W}$. An alternative approach is to work with the closure, within $\mathscr{P}$, of shock wave solutions $\left(U_{-}, U_{+}, s\right)$ with $U_{+} \neq U_{-}$. According to 2.6 , the set obtained is $\pi[\mathscr{W}]$, where $\pi: \mathscr{P}^{*} \rightarrow \mathscr{P}$ is the collapsing map. The alternative approach is equivalent to the present one if (a) $\pi[\mathscr{W}]$ is a smooth manifold and (b) $\pi$ establishes a diffeomorphism between $\mathscr{W}$ and $\pi[\mathscr{W}]$. To prove (a), we must construct local coordinates for $\pi[\mathscr{W}]$ near each of its points with $U_{+}=U_{-}$, which amounts to introducing $R$ and $\Omega$, i.e., by blowing up $\mathscr{P}$. Thus the alternative approach does not sidestep the blow-up process. Even so, one might prefer to work with $\pi[\mathscr{W}]$ after establishing its smoothness. This is reasonable if (b) holds, which means that $\Omega$ can be recovered from $\bar{U}$ and $s$. In some cases, however, $\Omega$ is not uniquely determined. In fact, if $s$ is an eigenvalue of $F^{\prime}(\bar{U})$ with geometric multiplicity $k \geqq 2$, then $\Omega$ can take any value in a $(k-1)$-dimensional projective space. Points with geometric multiplicity $k \geqq 2$ (called umbilic points) are generic in systems with many components $(n \geqq 4)$, and they occur in important models with $n=2$.

In the remainder of the paper we will introduce several geometric objects embedded in $\mathscr{W}$. Typically such as object is constructed in the manner that $\mathscr{W}$ is: it is the image under the identification map of the zero-set of a function defined on $\widehat{\mathscr{P}}$ (but not $\mathscr{P}^{*}$ ). As will be evident in each case, the zero-set is invariant under the antipodal transformation. Therefore it will prove convenient to perform calculations in $\widehat{\mathscr{P}}$. Implicit in the following is that all constructs in $\widehat{\mathscr{P}}$ are to be projected onto corresponding ones in $\mathscr{P}^{*}$.

Calculations with $\mathscr{F}$ are simplified by noting that $A(\bar{U}, 0, \Omega) r(\Omega)=F^{\prime}(\bar{U}) r(\Omega)$. Therefore alternative formulae for $\mathscr{F}$ are

$$
R \cdot \mathscr{F}(\bar{U}, R, \Omega, s)=-s\left[U_{+}-U_{-}\right]+F\left(U_{+}\right)-F\left(U_{-}\right)
$$

for $R \neq 0$ (Eqs. (2.6) being assumed) and

$$
\mathscr{F}(\bar{U}, 0, \Omega, s)=\left[-s+F^{\prime}(\bar{U})\right] r(\Omega)
$$

for $R=0$. Differentiating Eq. (2.13) shows that

$$
\begin{aligned}
& R \cdot d \mathscr{F}(\bar{U}, R, \Omega, s)+\mathscr{F} d R \\
& \quad=-\left[U_{+}-U_{-}\right] d s+\left[-s+F^{\prime}\left(U_{+}\right)\right] d U_{+}-\left[-s+F^{\prime}\left(U_{-}\right)\right] d U_{-}
\end{aligned}
$$

when $R \neq 0$. Furthermore, since $\mathscr{F}_{R}=0$ at $R=0$ by Lemma 2.5, Eq. (2.14) implies 
that

$$
d \mathscr{F}(\bar{U}, 0, \Omega, s)=-r(\Omega) d s+F^{\prime \prime}(\bar{U}) r(\Omega) d \bar{U}+\left[-s+F^{\prime}(\bar{U})\right] r^{\prime}(\Omega) d \Omega
$$

when $R=0$.

\section{The Characteristic Manifold $\mathscr{C}$}

The $R=0$ slice of $\mathscr{W}$, corresponding to rarefaction waves, plays a distinguished role. By Eq. (2.14), a point on this slice satisfies

$$
\left[-s+F^{\prime}(\bar{U})\right] r(\Omega)=0 .
$$

Thus $s$ is the speed for a characteristic direction associated with the system of conservation laws, and $r(\Omega)$ is a corresponding right eigenvector, which defines a hyperbolic mode that propagates at speed $s$ (cf. Eq. (2.3)).

Definition 3.1. The characteristic set $\mathscr{C}:=\mathscr{W} \cap \mathscr{P}_{0}^{*}$ is the exceptional subset of $\mathscr{W}$.

The characteristic set $\mathscr{C}$ generalizes the manifold introduced and studied by Palmeira [46] for a class of conservation laws with $n=2$ and quadratic fluxes. For a general system of two conservation laws, the characteristic manifold can be depicted within $\mathscr{P}_{0}^{*}$ (if the speed coordinate $s$ is suppressed); a schematic example is shown in Fig. 3.1. As we will see presently, each point $(\bar{u}, \bar{v})$ in the hyperbolic region of state space is the projection of two points of $\mathscr{C}$, while no portion of $\mathscr{C}$ lies above the elliptic region. The boundary separating these regions, which corresponds to coincidence of eigenvalues, is the projection of a fold locus.

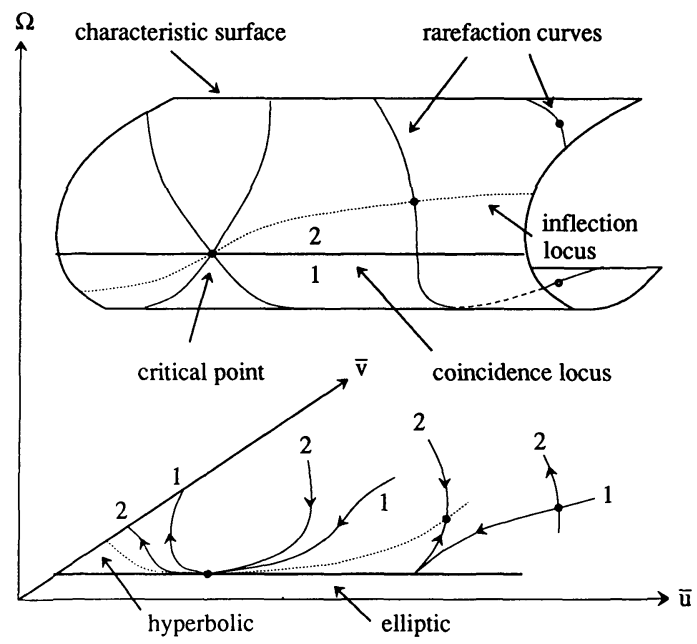

Fig. 3.1. A portion of a characteristic surface $\mathscr{C}$ for a system of two conservation laws, as embedded in $\mathscr{P}_{0}^{*}$ (with the speed coordinate $s$ suppressed). The surface folds along the coincidence locus, which projects onto the boundary between the hyperbolic and elliptic regions. The characteristic manifold is foliated by rarefaction curves, as discussed below. For the model shown, the rarefaction foliation has a saddle point singularity. (Adapted from ref. [23].) 


\section{Examples.}

1. For gas dynamics, $\mathscr{C}$ is defined by the equation $v_{+}=v_{-}=: \bar{v}$, so that $\mathscr{C}=\mathscr{C}_{\text {base }} \times \mathbf{R}$, where $\mathscr{C}_{\text {base }}$ consists of triples $(\bar{v}, \bar{v}, s)$ such that $s^{2}=-p^{\prime}(\bar{v})$. For a van der Waals gas with $T>1, p^{\prime}$ is strictly negative everywhere, so that $\mathscr{C}_{\text {base }}$ consists of the two disconnected curves $s= \pm \sqrt{-p^{\prime}(\bar{v})}$. In case $T<1$, we refer to Fig. 2.2, in which $\mathscr{C}_{\text {base }}$ is part of the diagonal $v_{+}=v_{-}$. If the local minimum and maximum of $p$ are attained at $\bar{v}=m$ and $\bar{v}=M$, respectively, then $\mathscr{C}_{\text {base }}$ consists of two disconnected branches corresponding to the intervals $0<\bar{v} \leqq m$ and $M \leqq \bar{v}$. At the critical temperature $T=1, \mathscr{C}_{\text {base }}$ consists of two curves that cross at $m=M$, so that it fails to be a manifold.

2. For quadratic models, $\mathscr{F}$ is independent of $R$, so that $(\bar{U}, R, \Omega, s) \in \mathscr{W}$ if and only if $(\bar{U}, 0, \Omega, s) \in \mathscr{C}$. Thus $\widehat{\mathscr{W}}$ is the product $\mathscr{C} \times \mathbf{R}$, and $\mathscr{W}$ is obtained by identifying each point $(\bar{U},-R,-\Omega, s)$ with $(U, R, \Omega, s)$. For the models studied by Palmeira [46], the characteristic manifold $\mathscr{C}$ is a cylinder embedded in $\mathscr{P}_{0}^{*}$ with two twists. An example of $\mathscr{C}$, parameterized by certain global coordinates $\kappa$ and $\Omega$, is shown in Fig. 3.2. See App. B of ref. [23] for more general quadratic models.

3. For KKIT models, $\mathscr{C}$ is the union of two submanifolds of $\mathscr{P}_{0}^{*}$ : the $(2 n-2)-$ dimensional submanifold $\mathscr{C}_{\text {contact }}$ of $\mathscr{W}_{\text {contact }}$ on which $\Phi^{\prime}(\bar{U}) r(\Omega)=0$ and $s=\Phi(\bar{U})$; and the $n$-dimensional submanifold $\mathscr{C}_{\text {ruled }}$ of $\mathscr{W}_{\text {ruled }}$ where $\bar{U}=\bar{\kappa} r(\Omega)$ for $\bar{\kappa} \in \mathbf{R}$ and $s=\Phi(\bar{U})+\Phi^{\prime}(\bar{U}) \bar{U}$, i.e., $s=g_{\Omega}^{\prime}(\bar{\kappa})$. These two submanifolds intersect when $\Phi^{\prime}(\bar{U}) \bar{U}=0$, so $\mathscr{C}$ fails to be a manifold. App. B of ref. [23] contains a discussion of the case $n=2$.

The following is a consequence of Lemma 2.7.

Theorem 3.2. Assume zero is a regular value of $\mathscr{F}$. Then the characteristic set $\mathscr{C}$ is an $n$-dimensional submanifold of the $(n+1)$-dimensional manifold $\mathscr{W}$.

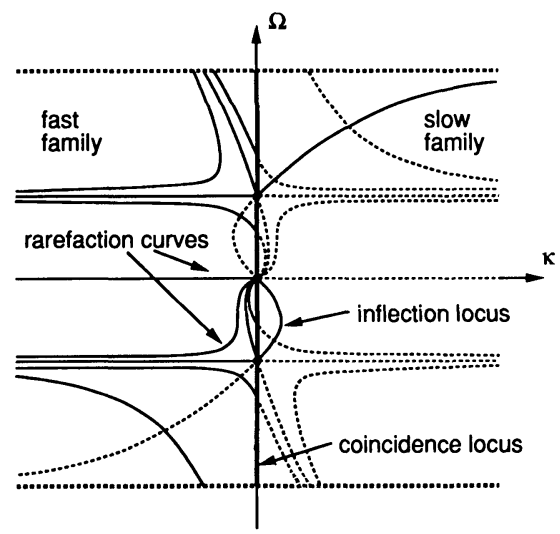

Fig. 3.2. The characteristic manifold $\mathscr{C}$ for the quadratic model $u_{t}+\left(3 u^{2} / 4+v^{2} / 2+v\right)_{x}=0$, $v_{t}+(u v-u)_{x}=0$. In terms of the global coordinates $\kappa \in \mathbf{R}$ and $\Omega \in[-\pi / 2, \pi / 2], \bar{u}=-4 \sin 2 \Omega-$ $\kappa \cos 2 \Omega$ and $\bar{v}=\sin 2 \Omega-(\kappa / 4) \cos 2 \Omega$. The edges $\Omega=-\pi / 2$ and $\Omega=\pi / 2$ are to be identified so that $\mathscr{C}$ is a cylinder. Also shown are other features defined below: the coincidence locus, $\kappa=0$; the sheets of $\mathscr{C}$ corresponding to the two eigenvalue families, slow and fast; typical rarefaction curves; three singularities of the rarefaction line field, one node and two saddle points; and the inflection locus 


\section{Remarks.}

1. More generally, the transversality of Lemma 2.7 implies that near each point of the diagonal $R=0, \mathscr{W}$ is locally the product $\mathscr{C} \times \mathbf{R}$, with the factor $\mathbf{R}$ corresponding to the $R$ coordinate.

2. The characteristic set can be defined independently of $\mathscr{W}$ as follows. Let $\mathscr{F}_{0}$ denote the restriction of $\mathscr{F}: \hat{\mathscr{P}} \rightarrow \mathbf{R}^{n}$ to the hypersurface $\hat{\mathscr{P}}_{0} \subset \hat{\mathscr{P}}$ where $R=0$, and let $\hat{\mathscr{C}} \subseteq \mathscr{P}_{0}^{*}$ be its zero-set. Then $\mathscr{C} \subseteq \mathscr{P}_{0}^{*}$ is the image of $\hat{\mathscr{C}}$ under the identification map $\sigma_{0}: \hat{\mathscr{P}}_{0} \rightarrow \mathscr{P}_{0}^{*}$. For $\mathscr{C}$ to be a submanifold of $\mathscr{P}_{0}^{*}$, it is sufficient that zero be a regular value of $\mathscr{F}_{0}$.

The structure of $\mathscr{C}$ reflects the behavior of the characteristic speeds for the system of conservation laws. According to Eq. (3.1), a state $\bar{U} \in \mathscr{U}$ is the projection of some point $(\bar{U}, 0, \Omega, s) \in \mathscr{C}$ if and only if $s$ is a (real) eigenvalue of $F^{\prime}(\bar{U})$. When $F^{\prime}(\bar{U})$ has $k$ real eigenvalues, all distinct, there are $k$ distinct points $\left(\bar{U}, 0, \Omega_{i}, s_{i}\right) \in \mathscr{C}$ that project onto $\bar{U}$, and $\mathscr{C}$ provides a $k$-fold covering for a sufficiently small neighborhood of $\bar{U}$ and $\mathscr{U}$. The case of coinciding real eigenvalues is addressed in the next result.

Lemma 3.3. Assume that $s$ is a real eigenvalue of $F^{\prime}(\bar{U})$ with corresponding eigenvector $r(\Omega)$. Then $s$ has algebraic multiplicity greater than 1 if and only if the $n \times n$ matrix $\left(\mathscr{F}_{\Omega}, \mathscr{F}_{s}\right)$ is singular at $(\bar{U}, 0, \Omega, s)$.

Furthermore, $s$ has algebraic multiplicity 2 if and only if $\left(\mathscr{F}_{\Omega}, \mathscr{F}_{s}\right)$ has rank $n-1$ and $\operatorname{lr}^{\prime}(\Omega) \dot{\Omega} \neq 0$ whenever $l$ and $(\dot{\Omega}, \dot{s})$ are nonzero left and right null vectors of $\left(\mathscr{F}_{\Omega}, \mathscr{F}_{s}\right)$, respectively. In this instance, s has geometric multiplicity 1 if and only if $\dot{s} \neq 0$.

Proof. Let $\tilde{e}_{2}, \ldots, \tilde{e}_{n}$ span the tangent space of $S^{n-1}$ at $\Omega$; define $e_{1}:=r(\Omega)$ and $e_{i}:=r^{\prime}(\Omega) \tilde{e}_{i}$ for $i=2, \ldots, n$. Because $F^{\prime}(\bar{U}) r(\Omega)=\operatorname{sr}(\Omega)$, the matrix of $-s+F^{\prime}(\bar{U})$ with respect to the basis $e_{1}, \ldots, e_{n}$ is

$$
B=\left(\begin{array}{ll}
0 & d \\
0 & D
\end{array}\right),
$$

where $d$ is an $(n-1)$-component row vector and $D$ is an $(n-1) \times(n-1)$ matrix. According to Eq. (2.16), $\mathscr{F}_{\Omega}=\left[-s+F^{\prime}(\bar{U})\right] r^{\prime}(\Omega)$ and $\mathscr{F}_{s}=-r(\Omega)$. Therefore $\left(\mathscr{F}_{\Omega}, \mathscr{F}_{\mathrm{s}}\right)$ has matrix

$$
C=\left(\begin{array}{cc}
-1 & d \\
0 & D
\end{array}\right)
$$

with respect to the basis $(0,1),\left(\tilde{e}_{2}, 0\right), \ldots,\left(\tilde{e}_{n}, 0\right)$ for its domain and the basis $e_{1}, \ldots, e_{n}$ for its range. The first statement of the lemma follows immediately: $s$ is a multiple eigenvalue of $F^{\prime}(\bar{U})$ if and only if $D$ is singular, i.e., $C$ is singular.

Suppose that $s$ has algebraic multiplicity 2. Then $D$ has rank $n-2$, so $C$ has rank $n-1$. Let $(\dot{\Omega}, \dot{s}) \neq 0$ span the kernel of $\left(\mathscr{F}_{\Omega}, \mathscr{F}_{s}\right)$; notice that $\dot{\Omega} \neq 0$ since $\mathscr{F}_{s} \neq 0$. Hence we can assume that $\tilde{e}_{2}=\dot{\Omega}$ and write

$$
\left(\begin{array}{l}
d \\
D
\end{array}\right)=\left(\begin{array}{ll}
\dot{s} & g \\
0 & f \\
0 & E
\end{array}\right),
$$

where $f$ and $g$ are $(n-2)$-component row vectors and $E$ is an $(n-2) \times(n-2)$ matrix; 
$E$ is nonsingular because $C$ has rank $n-1$. Expressed in the basis dual to $e_{1}, \ldots, e_{n}$, a left null vector $l$ of $\left(\mathscr{F}_{\Omega}, \mathscr{F}_{s}\right)$ is a nonzero multiple of $\left(0,1,-f E^{-1}\right)$. Therefore $\operatorname{lr}^{\prime}(\Omega) \dot{\Omega}=l e_{2} \neq 0$.

Conversely, suppose that $\left(\mathscr{F}_{\Omega}, \mathscr{F}_{s}\right)$ has rank $n-1$, with $(\dot{\Omega}, \dot{s}) \neq 0$ in its kernel. As before we can assume that $\tilde{e}_{2}=\dot{\Omega}$ and that Eq. (3.4) holds. If $s$ has algebraic multiplicity greater than 2 , then $E$ is singular, say $m E=0$ with $m \neq 0$. Thus there is a left null vector $l \neq 0$ of $C$, represented in the dual basis by $(0,0, m)$, such that $\operatorname{lr}^{\prime}(\Omega) \dot{\Omega}=l e_{2}=0$.

Finally, when $s$ has algebraic multiplicity 2, Eqs. (3.2) and (3.4) show that $s$ has geometric multiplicity 1 if and only if $\dot{s} \neq 0$.

Coincidence of eigenvalues is significant in the theory of conservation laws. For hyperbolic conservation laws, where the eigenvalues are real, coincidence corresponds to loss of strict hyperbolicity. For mixed-type conservation laws, the locus of coinciding eigenvalues separates regions with different hyperbolic-elliptic character. Lemma 3.3 suggests a definition.

Definition 3.4. The coincidence locus $\mathscr{E}$ is the set of points $(\bar{U}, 0, \Omega, s) \in \mathscr{C}$ where $s$ is a multiple eigenvalue of $F^{\prime}(\bar{U})$, i.e., the matrix $\left(\mathscr{F}_{\Omega}, \mathscr{F}_{s}\right)$ is singular. A point of $\mathscr{E}$ is called an umbilic point if $s$ has geometric multiplicity greater than 1.

Typically, $\mathscr{E}$ is an $(n-1)$-dimensional submanifold of $\mathscr{C}$. For this it is sufficient that zero be a regular value of the function $\operatorname{det}\left(\mathscr{F}_{\Omega}, \mathscr{F}_{s}\right)$.

Examples.

1. For gas dynamics, there is no coincidence locus if $T>1$. If $T<1, \mathscr{E}$ consists of two straight lines with $s=0, \bar{u}$ arbitrary, and either $v_{-}=m=v_{+}$or $v_{-}=M=v_{+}$.

2. In (nondegenerate) quadratic models with $n=2$, the projection of $\mathscr{E}$ onto $\mathscr{U}$ is a conic section [23]. It can be shown that $\mathscr{C}$ is a ruled surface embedded in $\mathscr{P}_{0}^{*}$ with zero, one, or two twists according $\mathrm{s}$ the projected coincidence locus is a hyperbola, a parabola, or an ellipse.

3. For KKIT models, the coincidence locus $\mathscr{E}$ contains $\mathscr{C}_{\text {ruled }} \cap \mathscr{C}_{\text {contact }}$. If $n \geqq 3$, then $\mathscr{C}_{\text {contact }} \subseteq \mathscr{E}$.

The characteristic manifold $\mathscr{C}$, regarded as covering state space $\mathscr{U}$, has sheets corresponding to eigenvalue families. One geometric interpretation for eigenvalue coincidence is that these sheets glue along $\mathscr{E}$. More precisely, we shall show that the projection from $\mathscr{C}$ to $\mathscr{U}$ folds along $\mathscr{E}$.

Definition 3.5. Let $\rho: \mathscr{C} \rightarrow \mathscr{U}$ denote the projection $(\bar{U}, 0, \Omega, s) \rightarrow \bar{U}$ from $\mathscr{P}_{0}^{*}$ to $\mathscr{U}$, as restricted to $\mathscr{C} \subset \mathscr{P}_{0}^{*}$.

Recall that a point $p \in X$ is a fold point for a smooth map $f: X \rightarrow Y$ between $m$-dimensional manifolds $X$ and $Y$ when there are local coordinates $\left(x_{1}, \ldots, x_{m}\right)$ for $X$ near $p$ and $\left(y_{1}, \ldots, y_{m}\right)$ for $Y$ near $f(p)$ in terms of which $f$ takes the form $\left(x_{1}, \ldots, x_{m-1}, x_{m}\right) \mapsto\left(x_{1}, \ldots, x_{m-1}, x_{m}^{2}\right)$. This is equivalent to the following conditions: (a) $f^{\prime}(p)$ has rank $m-1$; and (b) $l f^{\prime \prime}(p) r^{2} \neq 0$, where $l$ and $r$ are nonzero left and right null vectors of $f^{\prime}(p)$, respectively. See, e.g., ref. [17].

The following lemma characterizes fold points for a map $f$ that is the restriction of another map $g$ to a submanifold. 
Lemma 3.6. Suppose that $g: X \times \mathbf{R}^{m} \rightarrow Y$ is a smooth map for which $f(x)=g\left(x, z_{0}\right)$; let $h: X \times \mathbf{R}^{m} \rightarrow Y \times R^{m}$ with $h(x, z):=(g(x, z), z)$. Then $p \in X$ is a fold point for $f$ if and only if $\left(p, z_{0}\right)$ is a fold point for $h$.

Proof. Elements of the kernel of $h^{\prime}\left(p, z_{0}\right)$ are $(r, 0)$ where $f^{\prime}(p) r=0$, and left null vectors of $h^{\prime}\left(p, z_{0}\right)$ are $\left(l,-\lg _{z}\left(p, z_{0}\right)\right)$, where $l f^{\prime}(p)=0$. Furthermore, it is easy to check that $\left(l,-\lg _{z}\left(p, z_{0}\right)\right) h^{\prime \prime}\left(p, z_{0}\right)(r, 0)^{2}=l f^{\prime \prime}(p) r^{2}$. Therefore $f$ folds at $p$ if and only if $h$ holds at $\left(p, z_{0}\right)$.

Theorem 3.7. The map $\rho: \mathscr{C} \rightarrow \mathscr{U}$ is singular at $p:=(\bar{U}, 0, \Omega, s) \in \mathscr{C}$ if and only $p \in \mathscr{E}$, i.e., the eigenvalue s of $F^{\prime}(\bar{U})$ has algebraic multiplicity greater than 1 . Moreover, $p \in \mathscr{E}$ is a fold point for $\rho$ if and only if s has algebraic multiplicity 2 and geometric multiplicity 1.

Proof. A tangent vector $(\dot{\bar{U}}, 0, \dot{\Omega}, \dot{s})$ of $\mathscr{P}_{0}^{*}$ is tangent to $\mathscr{C}$ if and only if $\mathscr{F}_{\bar{U}} \dot{\bar{U}}+$ $\mathscr{F}_{\Omega} \dot{\Omega}+\mathscr{F}_{s} \dot{s}=0$, while its image under $\rho^{\prime}(p)$ is $\dot{\bar{U}}$. Therefore $(\dot{\bar{U}}, 0, \dot{\Omega}, \dot{s})$ belongs to the kernel of $\rho^{\prime}(p)$ if and only if $\dot{\bar{U}}=0$ and $(\dot{\Omega}, \dot{s})$ is in the kernel of $\left(\mathscr{F}_{\Omega}, \mathscr{F}_{s}\right)$. The first conclusion of the theorem follows from Def. 3.4 and Lemma 3.3.

To determine when $p \in \mathscr{E}$ is a fold point for $\rho$, consider the map $\psi: \mathscr{P}_{0}^{*} \rightarrow \mathscr{U} \times \mathbf{R}^{n}$ carrying $(\bar{U}, 0, \Omega, s)$ to $(\bar{U}, \mathscr{F}(\bar{U}, 0, \Omega, s))$. Then $p$ is a fold point for $\rho$ if and only if it is one for $\psi$. Indeed, because $\mathscr{C}$ is the submanifold of $\mathscr{P}_{0}^{*}$ where $\mathscr{F}=0$, there are coordinates $(x, y)$ for $\mathscr{P}_{0}^{*}$ near $p$ in which $\mathscr{C}$ is given by $y=0$ and $\psi$ takes the form $(x, y) \mapsto(\tilde{\rho}(x, y), y)$. In these coordinates, $\rho$ carries $x$ to $\tilde{\rho}(x, 0)$, so the result follows from Lemma 3.6.

Applying this same lemma to $\psi$ shows that $(\bar{U}, 0, \Omega, s)$ is a fold point for $\psi$ if and only if $(\Omega, s)$ is a fold point for the map $\phi:(\Omega, s) \mapsto \mathscr{F}(\bar{U}, 0, \Omega, s)$. Thus $p$ is a fold point of $\rho$ if and if (a) $\left(\mathscr{F}_{\Omega}, \mathscr{F}_{s}\right)$ has rank $n-1$; and (b) $l \phi^{\prime \prime}(\Omega, s) r^{2} \neq 0$, where $l$ and $r$ are nonzero left and right null vectors for $\left(\mathscr{F}_{\Omega}, \mathscr{F}_{s}\right)$, respectively. As in Lemma 3.3, $r=(\dot{\Omega}, \dot{s})$; since $\mathscr{F}_{\Omega}=\left[-s+F^{\prime}(\bar{U})\right] r^{\prime}(\Omega), l$ is a left null vector of $-s+F^{\prime}(\bar{U})$. Consequently, $l \phi^{\prime \prime}(\Omega, s) r^{2}=-2 \dot{s} l r^{\prime}(\Omega) \dot{\Omega}$, as a simple calculation shows. In other words, condition (b) holds if and only if $\dot{s} \neq 0$ and $\operatorname{lr}^{\prime}(\Omega) \Omega \neq 0$. To complete the proof, we invoke Lemma 3.3.

Remark. Consider the projection $\rho: \mathscr{C} \rightarrow \mathscr{U}$ when $n=2$. By Whitney's theorem (see, e.g., ref. [17]), singularities of maps between 2-dimensional manifolds are generically folds and simple cusps. Nevertheless, $\rho$ cannot exhibit a simple cusp singularity, since otherwise there would be points in $\mathscr{U}$ having three distinct preimages in $\mathscr{C}$.

Slicing the characteristic manifold $\mathscr{C}$ by fixing $\Omega$ yields a curve, called a fixedeigenvector curve, that is defined implicity by $\mathscr{F}(\bar{U}, 0, \Omega, s)=0$. The projection of such a slice onto the state space $\mathscr{U}$, also called a fixed-eigenvector curve, satisfies a system of $n-1$ equations $\mathscr{G}(\bar{U}, \Omega)=0$ obtained by eliminating the variable $s$. Thus the projected slices form a family of curves in $\mathscr{U}$ parameterized by $\Omega$. The envelope of this family is the projection of the coincidence locus $\mathscr{E}$; this is because $\left(\mathscr{F}_{\Omega}, \mathscr{F}_{s}\right)$ is singular if and only if $\mathscr{G}_{\Omega}$ is. Fixed-eigenvector curves are useful in the analysis of mixed-type conservation laws, such as those governing multiphase flow [43].

\section{Examples.}

1. Since $\cot \Omega=-s$ in gas dynamics, a fixed-eigenvector curve is a level curve of $s$ in $\mathscr{C}$. As $\mathscr{C}=\mathscr{C}_{\text {base }} \times \mathbf{R}$, a fixed-eigenvector curve is a union of lines, on which $p^{\prime}(\bar{v})=$ const., each line being parameterized by $\bar{u}$. (For a van der Wasls gas, a 
fixed-eigenvector curve is the union of at most three lines.) Thus $\mathscr{C}$ is ruled by fixed-eigenvector curves.

2. For (nondegenerate) quadratic models, $\mathscr{C}$ is a ruled surface, the rules being slices at fixed $\Omega$, which are therefore the fixed-eigenvector curves. Projected onto $\mathscr{U}$, a fixed-eigenvector curve is a straight line (called a characteristic line in Ref. [23]) that is tangent to the boundary of the elliptic region in state space.

3. For KKIT models, the fixed-eigenvector curves within $\mathscr{C}_{\text {ruled }}$ are the rules, which project onto lines through the origin in $\mathscr{U}$.

\section{The Rarefaction Foliation}

A rarefaction wave is a solution $U(x, t)=\tilde{U}(x / t)$ satisfying Eq. (2.3); such a solution is constructed as follows. Suppose that $F^{\prime}\left(U_{*}\right)$ has $k$ real eigenvalues at some point $U_{*} \in \mathscr{U}$, and that these real eigenvalues are distinct. Then there are smooth functions $\lambda_{i}$ and vector fields $r_{i}, i=1, \ldots, k$, defined on an open neighborhood $\mathcal{O}$ of $U_{*}$, such that $r_{i}(U)$ is an eigenvector of $F^{\prime}(U)$ with corresponding eigenvalue $\lambda_{i}(U)$ for all $U \in \mathcal{O}$. For each family $i$, integrating the ordinary differential equation

$$
\tilde{U}^{\prime}=r_{i}(\tilde{U})
$$

yields a rarefaction curve in $\mathscr{U}$ through each point of $\mathcal{O}$. Such a curve satisfies Eq. (2.3) if it is reparameterized so that $\lambda_{i}(\tilde{U})=x / t$. This is possible provided that the genuine nonlinearity condition,

$$
\lambda_{i}^{\prime}(U) \cdot r_{i}(U) \neq 0,
$$

holds all along the curve, meaning that the characteristic speed varies monotonically along it.

This construction of rarefaction curves in $\mathscr{U}$ fails, however, when the real eigenvalues are not distinct. This difficulty is remedied [46] by considering a line field on the characteristic manifold $\mathscr{C}$. Because multiple sheets of $\mathscr{C}$ lie over $\mathscr{U}$, the several families of rarefaction curves in $\mathscr{U}$ arise by projecting the integral curves of the single line field on $\mathscr{C}$.

Recall that $\mathscr{P}_{0}^{*}$ denotes the exceptional hypersurface $R=0$ of $\mathscr{P}^{*}$, viz., $\mathscr{P}_{0}^{*}=$ $\mathscr{U} \times\{0\} \times \mathbf{R} P^{n-1} \times \mathbf{R}$. As mentioned previously, the elements of the factor $\mathbf{R} P^{n-1}$ are lines tangent to $\mathscr{U}:$ if $(\bar{U}, 0, \Omega, s) \in \mathscr{P}_{0}^{*}$, then $r(\Omega)$ spans a line in $T_{\bar{U}} \mathscr{U}$, the tangent space of $\mathscr{U}$ at $\bar{U}$. Notice that for any vector $(\overline{\bar{U}}, 0, \dot{\Omega}, \dot{s})$ tangent to $\mathscr{P}_{0}^{*}$ at $(\bar{U}, 0, \Omega, s)$, the component $\dot{\bar{U}}$ also belongs to $T_{\bar{U}} \mathscr{U}$. Furthermore, the set of vectors $(\dot{\bar{U}}, 0, \dot{\Omega}, \dot{s})$ for which $\dot{\bar{U}}$ lies in the line spanned by $r(\Omega)$ forms an $(n+1)$-dimensional plane. In other words, there is a natural field $\mathscr{T}$ of $(n+1)$-dimensional tangent planes on $\mathscr{P}_{0}^{*}$ : the vector $(\dot{\bar{U}}, 0, \dot{\Omega}, \dot{s})$ at the point $p:=(\bar{U}, 0, \Omega, s)$ belongs to the plane $\mathscr{T}_{p}$ provided that $\dot{\bar{U}}$ is parallel to $r(\Omega)$. At a point $p$ on the $n$-dimensional submanifold $\mathscr{C} \subset \mathscr{P}{ }_{0}^{*}$, the plane $\mathscr{T}_{p}$ intersects $T_{p} \mathscr{C}$ along a line (unless $\mathscr{T}_{p}$ and $T_{p} \mathscr{C}$ are not in general position).

Definition 4.1. The (possibly singular) line field on $\mathscr{C}$ induced by the plane field $\mathscr{T}$ is called the rarefaction line field. A rarefaction curve in $\mathscr{C}$ is an integral curve of the rarefaction line field. 
Thus the rarefaction line field contains vectors $(\dot{\bar{U}}, 0, \dot{\Omega}, \dot{s})$ tangent to $\mathscr{C}$ such that $\bar{U}$ is parallel to $r(\Omega)$. We emphasize that information regarding the conservation laws is encoded in the manifold $\mathscr{C}$ and its embedding in $\mathscr{P}_{0}^{*}$, not in the plane field $\mathscr{T}$.

Example. If we suppress the trivial factor $\mathbf{R}$ corresponding to the speed $s, \mathscr{P}_{0}^{*}$ is essentially the canonical line bundle $\mathscr{U} \times \mathbf{R} P^{n-1}$. This space is depicted in Fig. 4.1 for the case $n=2$. The vector $(\dot{\bar{u}}, \dot{\bar{v}}, \dot{\Omega})$ at $p:=(\bar{u}, \bar{v}, \Omega)$ lies in $\mathscr{T}_{p}$ provided that $-\sin \Omega \dot{\bar{u}}+\cos \Omega \dot{\bar{v}}=0$.

Proposition 4.2. The singularities of the rarefaction line field occur at the points of $\mathscr{C}$ where $\left(\frac{1}{2} \mathscr{F}_{\bar{U}} r(\Omega), \mathscr{F}_{\Omega}, \mathscr{F}_{s}\right)$ has rank less than $n$. In particular, they belong to the coincidence locus $\mathscr{E} \subseteq \mathscr{C}$.

Proof. With $p:=(\bar{U}, 0, \Omega, s)$, a vector in $\mathscr{T}_{p}$ takes the form $\left(\frac{1}{2} \dot{R} r(\Omega), 0, \dot{\Omega}, \dot{s}\right)$; this vector is tangent to $\mathscr{C}$ if and only if

$$
\frac{1}{2} \mathscr{F}_{\bar{U}} r(\Omega) \dot{R}+\mathscr{F}_{\Omega} \dot{\Omega}+\mathscr{F}_{s} \dot{s}=0 .
$$

Singularities of the rarefaction line field occur when this linear system does not have full rank, i.e., $\left(\frac{1}{2} \mathscr{F}_{\vec{U}} r(\Omega), \mathscr{F}_{\Omega}, \mathscr{F}_{s}\right)$ has rank less less than $n$. A singularity belongs to $\mathscr{E}$ because $\left(\mathscr{F}_{\Omega}, \mathscr{F}_{s}\right)$ must be singular if $\left(\frac{1}{2} \mathscr{F}_{\bar{U}} r(\Omega), \mathscr{F}_{\Omega}, \mathscr{F}_{s}\right)$ is to be rank deficient.

Definition 4.3. The locus of rarefaction singularities $\mathscr{B}_{0}$ is the set of points at which the rarefaction line field is singular, i.e., $\left(\frac{1}{2} \mathscr{F}_{\bar{U}} r(\Omega), \mathscr{F}_{\Omega}, \mathscr{F}_{s}\right)$ is rank deficient.

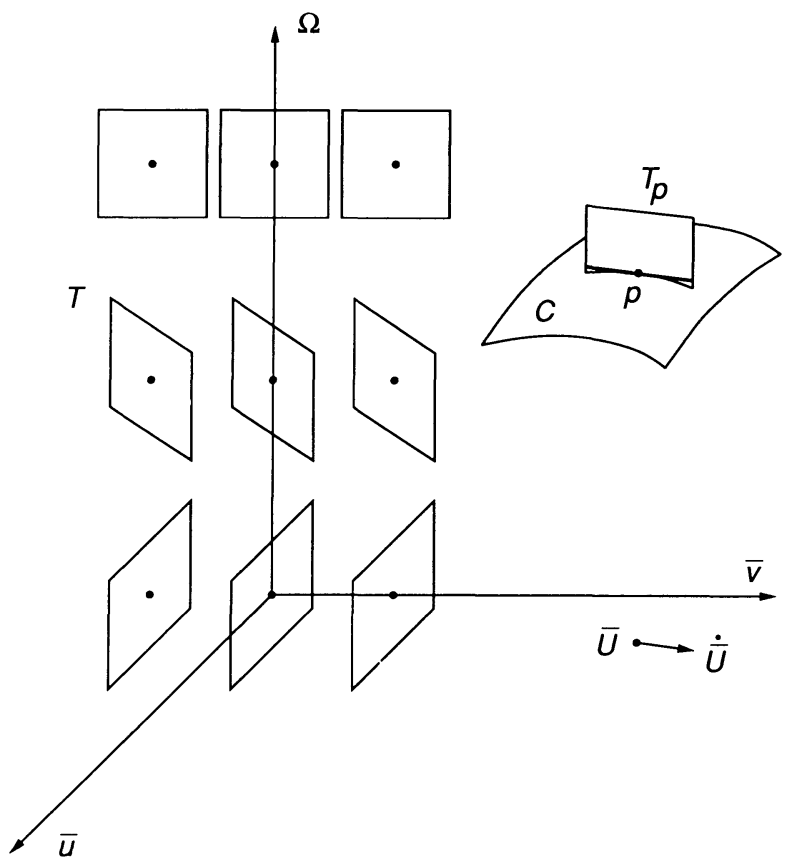

Fig. 4.1. The exceptional hypersurface $\mathscr{P}_{0}^{*}$ for $n=2$, with the speed coordinate $s$ suppressed. The field of tangent planes $\mathscr{T}$ is illustrated. The plane through $p:=(\bar{u}, \bar{v}, \Omega)$ projects to the line through $\bar{U}=(\bar{u}, \bar{v})$ that lies at angle $\Omega$ relative to the $\bar{u}$-axis. The intersection of $\mathscr{T}_{p}$ with the tangent plane $T_{p} \mathscr{C}$ is a line; such lines constitute the rarefaction line field 
Corollary 4.4. Rarefaction curves consitute a one-dimensional foliation of $\mathscr{C} \backslash \mathscr{B}_{0}$.

The condition that characterizes singularities represents one additional constraint on points of $\mathscr{E}$. Assuming certain nondegeneracy conditions, $\mathscr{B}_{0}$ has dimension $n-2$.

\section{Examples.}

1. For gas dynamics, $\mathscr{C}$ is defined by $s^{2}=-p^{\prime}(\bar{v})$, so that the vector $(\dot{\bar{v}}, \dot{\bar{v}}, \dot{s}, \dot{\bar{u}})$ is tangent of $\mathscr{C}$ if and only if $2 s \dot{s}+p^{\prime \prime}(\bar{v}) \dot{\bar{v}}=0$. Since $\cot \Omega=-s, \dot{\bar{U}}=(\dot{\bar{u}}, \dot{\bar{v}})$ is parallel to $r(\Omega)$ when $\dot{\bar{u}}+s \dot{\bar{v}}=0$. These two linear equations for $\dot{\bar{v}}, \dot{s}$, and $\dot{\bar{u}}$ are linearly dependent if and only if $s=0$ and $p^{\prime \prime}(\bar{v})=0$; thus singularities of the characteristic foliation on $\mathscr{C}$ occur at points where both $p^{\prime}(\bar{v})$ and $p^{\prime \prime}(\bar{v})$ vanish. There are no singularities for a van der Waals gas unless $T=1$.

2. Nondegenerate quadratic models with $n=2$ have one, two, or three singularity points on $\mathscr{E}$, which correspond to the roots of a cubic polynomial $[46,23]$. For the models studied ref. [46], a singularity is either a node or a saddle. An example is shown in Fig. 3.2.

3. On the manifold $\mathscr{C}_{\text {contact }}$ of a KKIT model, $\Phi^{\prime}(\bar{U}) r(\Omega)=0$, so that if $\dot{\bar{U}}=\frac{1}{2} \dot{R} r(\Omega)$, then $\Phi^{\prime}(\bar{U}) \dot{\bar{U}}=0$. Therefore a rarefaction curve is contained in a locus of contact discontinuities $\Phi(\bar{U})=$ const. A vector tangent to $\mathscr{C}_{\text {ruled }}$ satisfies $\bar{U}=\dot{\bar{\kappa}} r(\Omega)+$ $\bar{\kappa} r^{\prime}(\Omega) \dot{\Omega}$; for $\bar{U}$ to be parallel to $r(\Omega), \dot{\Omega}$ must vanish. Thus a rarefaction curve on this surface is a fixed-eigenvector curve $\Omega=$ const. Since $s=g_{\Omega}^{\prime}(\bar{\kappa})$, rarefaction curves mimic those for the scalar conservation law $\kappa_{t}+g_{\Omega}(\kappa)_{x}=0$. A simple calculation shows that the locus of rarefaction singularities contains the coincidence locus and, if $n \geqq 3$, the whole of $\mathscr{C}_{\text {contact }}$.

An integral curve for the rarefaction line field can be obtained, locally, by integrating a vector field. Consider the (local) vector field

$$
\begin{aligned}
\dot{\bar{U}} & =\operatorname{det}\left(\mathscr{F}_{\Omega}, \mathscr{F}_{s}\right) r(\Omega), \\
\left(\begin{array}{c}
\dot{\Omega} \\
\dot{s}
\end{array}\right) & =-\operatorname{adj}\left(\mathscr{F}_{\Omega}, \mathscr{F}_{s}\right) \mathscr{F}_{\bar{U}} r(\Omega),
\end{aligned}
$$

where $\operatorname{adj} B$ denotes the classical adjoint of the matrix $B$, so that $B \operatorname{adj} B=(\operatorname{det} B) I$. This field belongs to the rarefaction line field since it satisfies the tangency condition $\mathscr{F}_{\bar{U}} \dot{\bar{U}}+\mathscr{F}_{\Omega} \dot{\Omega}+\mathscr{F}_{s} \dot{s}=0$. Furthermore, its critical points occur precisely where the line field is singular; in fact, the matrix $\left(\frac{1}{2} \mathscr{F}_{\bar{U}} r(\Omega), \mathscr{F}_{\Omega}, \mathscr{F}_{s}\right)$ fails to have full rank if and only if

$$
\begin{aligned}
\operatorname{det}\left(\mathscr{F}_{\Omega}, \mathscr{F}_{s}\right) & =0, \\
\operatorname{adj}\left(\mathscr{F}_{\Omega}, \mathscr{F}_{s}\right) \mathscr{F}_{\bar{U}} r(\Omega) & =0 .
\end{aligned}
$$

Equation (4.4) does not, however, define a global vector field on $\mathscr{C}$ or $\mathscr{P}_{0}^{*}$, since it is not invariant under the antipodal transformation.

Rarefaction curves in $\mathscr{U}$, which are used to construct rarefaction waves, are the projections of rarefaction curves in $\mathscr{C}$ under the map $\rho: \mathscr{C} \rightarrow \mathscr{U}$. Away from the coincidence locus, $\rho$ is a covering map, so that the projected curves are smooth; at the coincidence locus, however, the projection introduces singularities.

Proposition 4.5. Suppose that $p \in \mathscr{E} \backslash \mathscr{B}_{0}$ is a fold point for the map $\rho: \mathscr{C} \rightarrow \mathscr{U}$. Then the rarefaction curve through $p$ is transverse to $\mathscr{E}$, and its projection onto $\mathscr{U}$ has a cusp. 
Proof. By Eq. (4.4), a tangent to the rarefaction curve at $p$ has $\dot{\bar{U}}=0$, so that it spans the kernel of $\rho^{\prime}(p)$. Since $p$ is a fold point, this vector and members of $T_{p} \mathscr{E}$ together span $T_{p} \mathscr{C}$. If $\alpha \mapsto q(\alpha)$ parameterizes the rarefaction curve, with $q(0)=p$ and $q^{\prime}(0) \neq 0$ in the kernel of $\rho^{\prime}(p)$, then $\left(\rho^{\circ} q\right)^{\prime}(0)=0$ but $\left(\rho^{\circ} q\right)^{\prime \prime}(0) \neq 0$, as follows from the normal form of $\rho$ at the fold point $p$.

In the classical theory, it is sometimes useful to regard rarefaction curves of a given family as level curves of $n-1$ real-valued functions defined on $\mathscr{U}$, the so-called Riemann invariants. For instance, consider a strictly hyperbolic system of two conservation laws. Locally there is a Riemann invariant $w_{i}$ for each of the two families, $i=1,2$. In this case, the characteristic manifold $\mathscr{C}$ is a double covering of $\mathscr{U}$, which consists of two disjoint sheets $\mathscr{C}_{1}$ and $\mathscr{C}_{2}$ associated to families 1 and 2 , respectively. Therefore the two functions $w_{i}$ can be consolidated into a single function $w$ on $\mathscr{C}$ : define $w$ to be $w_{i}$ when it is restricted to $\mathscr{C}_{i}, i=1,2$.

A natural generalization of Riemann invariants in the present formalism is a function $w: \mathscr{C} \rightarrow \mathbf{R}^{n-1}$, the level curves of which are the rarefaction curves. To avoid trivialities, $w$ should not be locally constant anywhere [34]. (Strictly hyperbolic systems have Riemann invariants with nonvanishing gradients.) When $n=2$, such a function $w$ exists locally away from rarefaction singularities. For example, the two Riemann invariants defined by Kan [30] for symmetric Case IV quadratic models derive from a single function defined on $\mathscr{C}$. For Case III models, however, no global Riemann invariant $w$ exists because one of the rarefaction singularities is a node. (See Fig. 3.2.) More generally, a Riemann invariant can be constructed for a quadratic model so long as all rarefaction singularities are saddle points; this follows from the formulae in App. B of ref. [23].

\section{The Inflection Locus}

Return, for the moment, to the classical construction of rarefaction waves when the real eigenvalues of $F^{\prime}(U)$ are distinct, as described at the beginning of Sect. 4. To construct a rarefaction wave, the characteristic speed $\lambda_{i}$ must vary monotonically along the rarefaction curve in $\mathscr{U}$. Therefore points at which $\lambda_{i}$ reaches an extremum are important in solving Riemann problems. It proves simpler and more useful to find stationary points for $\lambda_{i}$. By definition, these are points where genuine nonlinearity [33] fails.

Proposition 5.1. Suppose that the real eigenvalues of $F^{\prime}(U)$ are distinct. Consider the graph of the characteristic speed $\lambda_{i}$ along an integral curve through $U$ for the eigenvector field $r_{i}$. Then the graph has a stationary point at $U$ if and only if $l F^{\prime \prime}(U) r^{2}=0$, where $l$ and $r:=r_{i}(U)$ are nonzero left and right null vectors of the matrix $-\lambda_{i}(U)+F^{\prime}(U)$, respectively.

Moreover, at such a stationary point, the graph of $\lambda_{i}$ has vanishing second derivative if and only if

$$
l F^{\prime \prime \prime}(U) r_{i}(U)^{3}+3 l F^{\prime \prime}(U) \cdot\left(r_{i}(U) \otimes r_{i}^{\prime}(U) r_{i}(U)\right)=0 .
$$

Proof. For simplicity, let a dot (temporarily) denote differentiation with respect to $U$ in the direction of $r_{i}(U)$. Differentiating the relation $F^{\prime}(U) r_{i}(U)=\lambda_{i}(U) r_{i}(U)$ yields

$$
F^{\prime \prime}(U) r_{i}(U)^{2}+\left[-\lambda_{i}(U)+F^{\prime}(U)\right] \dot{r}_{i}(U)=\dot{\lambda}_{i}(U) r_{i}(U) .
$$


Therefore $l F^{\prime \prime}(U) r_{i}(U)^{2}=\dot{\lambda}_{i}(U) l r_{i}(U)$. Notice that $l r_{i}(U) \neq 0$ because $\lambda_{i}(U)$ is a simple eigenvalue of $F^{\prime}(U)$. Hence $\dot{\lambda}_{i}(U)=0$ if and only if $l F^{\prime \prime}(U) r_{i}(U)^{2}=0$.

Differentiating Eq. (5.2) and evaluating at a point where $\dot{\lambda}_{i}(U)=0$ shows that

$$
\begin{aligned}
F^{\prime \prime \prime}(U) r_{i}(U)^{3} & +3 F^{\prime \prime}(U) \cdot\left(r_{i}(U) \otimes \dot{r}_{i}(U)\right) \\
+ & {\left[-\lambda_{i}(U)+F^{\prime}(U)\right] \ddot{r}_{i}(U)=\ddot{\lambda}_{i}(U) r_{i}(U) . }
\end{aligned}
$$

Thus, $\ddot{\lambda}_{i}(U)=0$ if and only if Eq. (5.1) holds.

A rarefaction curve in $\mathscr{C}$, projected onto the state space $\mathscr{U}$, provides a solution of Eq. (4.1), with $s$ corresponding to $\lambda_{i}$. Stationary points for $s$, regarded as a function along a rarefaction curve, occur where the line field lies in the hyperplane $d s=0$.

Lemma 5.2. At a point $(\bar{U}, 0, \Omega, s) \in \mathscr{C}$, there is a nonzero vector contained in both the rarefaction line field and the tangent hyperplane $d s=0$ if and only if the matrix $B_{0}(\bar{U}, \Omega, s):=\left(\frac{1}{2} \mathscr{F}_{\bar{U}} r(\Omega), \mathscr{F}_{\Omega}\right)$ is singular.

Proof. Let $\left(\frac{1}{2} \dot{R} r(\Omega), 0, \dot{\Omega}, \dot{s}\right) \neq 0$ belong to the line field. According to Eq. (4.3), $\dot{s}=0$ only if $(\dot{R}, \dot{\Omega})$ is a null vector for $B_{0}(\bar{U}, \Omega, s)$. Conversely, if $(\dot{R}, \dot{\Omega}) \neq 0$ is a null vector, then $\left(\frac{1}{2} \dot{R} r(\Omega), 0, \dot{\Omega}, 0\right) \neq 0$ is tangent to $\mathscr{C}$ and belongs to both the rarefaction line field and the hyperplane $d s=0$.

Theorem 5.3. At a point $p:=(\bar{U}, 0, \Omega, s) \in \mathscr{C} \backslash \mathscr{E}$, genuine nonlinearity fails at $\bar{U}$ for the characteristic family corresponding to $s$ if and only if the rarefaction line field is contained in the tangent hyperplane $d s=0$.

Proof. By Proposition 5.1 and the lemma, it suffices to show that $B_{0}(\bar{U}, \Omega, s)$ is singular if and only if $l F^{\prime \prime}(\bar{U}) r(\Omega)^{2}=0$, where $l \neq 0$ is a left null vector of $-s+F^{\prime}(\bar{U})$. Notice that $\mathscr{F}_{\bar{U}} r(\Omega)=F^{\prime \prime}(\bar{U}) r(\Omega)^{2}$ and $\mathscr{F}_{\Omega}=\left[-s+F^{\prime}(\bar{U})\right] r^{\prime}(\Omega)$, as seen from Eq. (2.16). Suppose that $B_{0}$ is singular; then there is an $l \neq 0$ such that $l B_{0}=0$, i.e., $l F^{\prime \prime}(\bar{U}) r(\Omega)^{2}=0$ and $l\left[-s+F^{\prime}(\bar{U})\right] r^{\prime}(\Omega)=0$. Since $p \notin \mathscr{E},\left[-s+F^{\prime}(\bar{U})\right] r^{\prime}(\Omega)$ has full rank $n-1$, so that $l$ must also be a null vector of $-s+F^{\prime}(\bar{U})$. Conversely if $l F^{\prime \prime}(\bar{U}) r(\Omega)^{2}=0$ and $l\left[-s+F^{\prime}(\bar{U})\right]=0$, then $l B_{0}=0$.

For scalar conservation laws (i.e., $n=1$ ), loss of genuine nonlinearity corresponds (typically) to an inflection point in the graph of the flux function. This suggests the following terminology.

Definition 5.4. The inflection locus $\mathscr{I}$ is the set of points of $\mathscr{C}$ where there is a nonzero vector contained in both the rarefaction line field and the tangent hyperplane $d s=0$, i.e., the matrix $B_{0}(\bar{U}, \Omega, s)=\left(\frac{1}{2} \mathscr{F}_{\bar{U}} r(\Omega), \mathscr{F}_{\Omega}\right)$ is singular.

In nondegenerate cases, $\mathscr{I}$ is an $(n-1)$-dimensional submanifold of $\mathscr{C}$. A sufficient condition is that zero be a regular value of the function $\operatorname{det} B_{0}$.

Examples.

1. In gas dynamics, the inflection locus is a union of lines, parameterized by $\bar{u}$, that are associated with points in the graph of $p$ where $p^{\prime}(\bar{v}) \leqq 0$ and $p^{\prime \prime}(\bar{v})=0$. When $p^{\prime}(\bar{v})<0$, such a point generates a pair of lines, corresponding to the two choices of sign for $s$. For a van der Waals gas with $T<1$, there is one pair of inflection lines, which is associated to the point labeled $\mathscr{I}$ in Fig. 2.2.

2. For (nondegenerate) quadratic models with $n=2$, the inflection locus is a union of curves in $\mathscr{C}$, which are either straight lines or are parameterized by $\Omega$ [23]. The 
inflection locus does not intersect itself even though its projection onto $\mathscr{U}$ can do so [46].

3. All points of the manifold $\mathscr{C}_{\text {contact }}$ in a KKIT model are inflection points. Indeed, $\Phi^{\prime}(\bar{U}) r(\Omega)=0$ and $s=\Phi(\bar{U})$, so that $s$ is constant along an integral curve. On $\mathscr{C}_{\text {ruled }}$, away from the coincidence locus, $\bar{U}=\bar{\kappa} r(\Omega)$ is an inflection point if and only if $g_{\Omega}^{\prime \prime}(\bar{\kappa})=0$, where $g_{\Omega}(\kappa):=\kappa \Phi(\kappa r(\Omega))$ is the associated scalar flux.

Usually a rarefaction curve intersects $\mathscr{I}$ transversally and the graph of $\lambda_{i}$ has an extremum. The exceptional points of $\mathscr{I}$ where transversality fails play an important role in wave curve bifurcation, as discovered by Furtado [14] (see Sect. 8). The following result motivates a general definition.

Lemma 5.5. Assume that $M: X \rightarrow L\left(\mathbf{R}^{m}, \mathbf{R}^{m}\right)$ is a smooth matrix-valued map defined on a manifold $X$; suppose that $\operatorname{det} M(x)=0$ for some $x \in X$. Then $x$ is a regular point for the map det $M: X \rightarrow \mathbf{R}$ if and only if (a) $M(x)$ has rank $m-1$ and (b) $l\left(M^{\prime}(x) \dot{x}\right) r \neq 0$ for some $\dot{x} \in T_{x} X$, where $l$ and $r$ are nonzero left and right null vectors of $M(x)$, respectively.

In this case, the subset of $X$ on which $M$ is singular is a smooth hypersurface in a neighborhood of $x$. Furthermore, the tangent space of this hypersurface at $x$ consists of vectors $\dot{x} \in T_{x} X$ such that $l\left(M^{\prime}(x) \dot{x}\right) r=0$.

Proof. The derivative of $\operatorname{det} M$ is $d \operatorname{det} M=\operatorname{tr}\{\operatorname{adj} M d M\}$. If (a) holds, then $l$ and $r$ can be normalized so that adj $M(x)=r l$ (outer product), so that $(\operatorname{det} M)^{\prime}(x) \dot{x}=$ $l\left(M^{\prime}(x) \dot{x}\right) r$. Therefore (b) implies that $d$ det $M \neq 0$ at $x$. Conversely, if $M(x)$ has rank less than $m-1$, then $\operatorname{adj} M=0$, so that $d \operatorname{det} M=0$ at $x$.

If $x$ is a regular point, then the implicit function theorem guarantees that the subset where $M$ is singular is a manifold near $x$. Furthermore, the tangent space at $x$ comprises vectors $\dot{x} \in T_{x} X$ such that $(\operatorname{det} M)^{\prime}(x) \dot{x}=0$.

Proposition 5.6. Suppose that $p:=(\bar{U}, 0, \Omega, s) \in \mathscr{I} \backslash \mathscr{E}$ is a regular point for the function $\operatorname{det} B_{0}$. Consider the graph of s along the rarefaction curve through $p$. This graph has vanishing second derivative at $p$ if and only if the rarefaction curve is tangent to $\mathscr{I}$ at $p$.

Proof. Let $l$ and $r:=(\dot{R}, \dot{\Omega})$ be nonzero left and right null vectors of $B_{0}(\bar{U}, \Omega, s)$, respectively. Notice that $\dot{R} \neq 0$, since otherwise both $r(\Omega)$ and $r^{\prime}(\Omega) \dot{\Omega}$ would be eigenvectors of $F^{\prime}(\bar{U})$ with eigenvalue $s$, contradicting $p \notin \mathscr{E}$. Also, the vector $\left(\frac{1}{2} \dot{R} r(\Omega), 0, \dot{\Omega}, 0\right)$ is tangent to the rarefaction curve at $p$. This curve is tangent to $\mathscr{I}$ if and only if $l \dot{B}_{0} r=0$, where $\dot{B}_{0}$ denotes $B_{0}^{\prime}$ applied to $\left(\frac{1}{2} \dot{R} r(\Omega), \dot{\Omega}, 0\right)$. As a simple calculation shows,

$$
l \dot{B}_{0} r=\frac{1}{4} \dot{R}^{2} l F^{\prime \prime \prime}(\bar{U}) r(\Omega)^{3}+\frac{3}{2} \dot{R} l F^{\prime \prime}(\bar{U}) \cdot\left(r(\Omega) \otimes r^{\prime}(\Omega) \dot{\Omega}\right) .
$$

The result follows from Eq. (5.1) if we can verify that $r^{\prime}(\Omega) \dot{\Omega}=\frac{1}{2} \dot{R} r_{i}^{\prime}(\bar{U}) r_{i}(\bar{U})$ when $r_{i}$ has been normalized such that $r_{i}(\bar{U})=r(\Omega)$. To see this, we recognize that both $\left(\dot{R}, r^{\prime}(\Omega) \dot{\Omega}\right)$ and $\left(1, \frac{1}{2} r_{i}^{\prime}(\bar{U}) r_{i}(\bar{U})\right)$ are right null vectors of the matrix $\left(\frac{1}{2} F^{\prime \prime}(\bar{U}) r(\Omega)^{2}\right.$, $\left.-s+F^{\prime}(\bar{U})\right)$, which has rank $n-1$. These two vectors are independent from the null vector $(0, r(\Omega))$, so they must be parallel.

Remark. The quantity $l \dot{B}_{0} r$ is defined intrinsically even though it involves second derivatives of $\mathscr{F}$; no choice of affine connection is needed. In fact, it is a generalization of the Hessian that derives from the intrinsic derivative of $B_{0}$ [17]. 
Definition 5.7. The exceptional inflection locus $\mathscr{H}_{0}$ consists of inflection points at which either (a) $p$ is not a regular point for the function $\operatorname{det} B_{0}$ or (b) $l \dot{B}_{0} r=0$, where $l$ and $r:=(\dot{R}, \dot{\Omega})$ are nonzero left and right null vectors of $B_{0}$, respectively, and $\dot{B}_{0}$ denotes $B_{0}^{\prime}$ applied to the vector $\left(\frac{1}{2} \dot{R} r(\Omega), \dot{\Omega}, 0\right)$.

The following consequence is important for wave curve bifurcation.

Corollary 5.8. Suppose that $p \in \mathscr{I}$ is a regular point of the function $\operatorname{det} B_{0}$. Then if $p \notin \mathscr{H}_{0} \cup \mathscr{B}_{0}$, the rarefaction curve through $p$ is transverse to $\mathscr{I}$ at $p$.

Examples.

1. In gas dynamics, the inflection lines associated to a point $\bar{v}$ consist entirely of exceptional points when $p^{\prime \prime \prime}(\bar{v})=0$.

2. For (nondegenerate) quadratic models with $n=2$, there are at most two exceptional inflection points.

3. All points of $\mathscr{C}_{\text {contact }}$ in a KKIT model are exceptional inflection points. On $\mathscr{C}_{\text {ruled }}$, away from the coincidence locus, an inflection locus is exceptional when $g_{\Omega}^{\prime \prime \prime}(\bar{\kappa})=0$.

The definition of $\mathscr{I}$ implies that singularities of the rarefaction foliation are points of inflection: $\mathscr{B}_{0} \subseteq \mathscr{I} \subset \mathscr{E}$. The converse is true at fold points.

Proposition 5.9. A fold point of the map $\rho: \mathscr{C} \rightarrow \mathscr{U}$ is a singularity of the rarefaction line field if and only if it belongs to the inflection locus.

Proof. We need only show that if the point is not a singularity, it is not a point of inflection; we invoke Theorem 3.7 and Lemma 3.3. At a fold point, the matrix $\left(\mathscr{F}_{\Omega}, \mathscr{F}_{s}\right)$ has rank $n-1$, and an element $(\dot{\Omega}, \dot{s}) \neq 0$ in its kernel has $\dot{s} \neq 0$. Since the vector $(0,0, \dot{\Omega}, \dot{s})$ belongs to the line field, the point is not on the inflection locus.

This result is significant for the existence of transitional rarefaction waves [23], which we now define. For strictly hyperbolic systems of conservation laws, the characteristic speeds $\lambda_{i}(U), i=1, \ldots, n$, can be arranged in increasing order. Thus a rarefaction wave has an associated family $i$. Furthermore, shock waves that conform to the Lax admissibility criterion can be classified according to family, and wave groups always comprise shock and rarefaction waves of the same family. When the system of conservation laws is not strictly hyperbolic, labeling of waves by family is still feasible, but wave groups involving several families can occur. For instance, a wave group is said to be transitional if it contains faster family waves situated on the left of slower family waves. A transitional rarefaction wave is a continuous transitional wave group. Such a wave is constructed by following a rarefaction curve in the direction of increasing $s$; for the wave to be transitional, the curve must pass from a sheet of $\mathscr{C}$ corresponding to a faster characteristic family to one with a slower family.

In the case $n=2$, there is a convenient analytical characterization of family: the family of a point of $\mathscr{C}$ is 1 or 2 according as $\Phi(\bar{U}, \Omega, s):=s-\frac{1}{2} \operatorname{tr} F^{\prime}(\bar{U})$ is negative or positive. The arguments of Proposition 5.9 applied to $\Phi$ show that a transitional rarefaction must cross the coincidence locus, where $\Phi$ vanishes, at either a singularity or a non-fold point. In fact, suppose that a rarefaction curve crosses the coincidence locus at a fold point that is not a rarefaction singularity. Then the derivative of $\Phi$ along this rarefaction curve, evaluated at the coincidence locus, is $\dot{\Phi}=\dot{s} \neq 0$; 
therefore if the rarefaction curve is traversed in the direction of increasing $s$, then $\Phi$ must increase across the curve. See ref. [23] for further discussion. We expect that this result holds as well when $n>2$.

\section{The Shock Foliation}

A fundamental step in solving Riemann problems is the construction of oneparameter families of shock waves $\left(U_{-}, U_{+}, s\right)$ with fixed left states $U_{-}$. Given a state $U_{0}$, the set of shock waves with $U_{-}=U_{0}$ is obtained by solving the $n$ equations

$$
-s\left[U-U_{0}\right]+F(U)-F\left(U_{0}\right)=0
$$

for the right state $U:=U_{+}$and shock speed $s$. This set is typically one-dimensional, although it can have several branches and can fail to be a one-dimensional submanifold (such as at self-intersections and isolated points).

Right states $U$ that satisfy Eq. (6.1) for some $s$ comprise a shock curve in $\mathscr{U}$ through the point $U_{0}$. Alternatively, the set of shock waves with fixed left state $U_{-}=U_{0}$ can be regarded as embedded in $\mathscr{P} *$; it consists of points $(\bar{U}, R, \Omega, s) \in \mathscr{W}$ such that

$$
\bar{U}-\frac{1}{2} \operatorname{Rr}(\Omega)=U_{0}
$$

for some fixed state $U_{0}$. Analogously, we can fix the right state $U_{+}=U_{0}$ instead, obtaining points such that

$$
\bar{U}+\frac{1}{2} \operatorname{Rr}(\Omega)=U_{0} .
$$

Definition 6.1. A fixed- $U_{-}$shock curve is a slice of $\mathscr{W}$ with fixed left state $U_{-} ; a$ fixed- $U_{+}$shock curve is a slice with fixed right state $U_{+}$.

\section{Examples.}

1. The shock curves for a van der Waals gas with $T<1$ can be determined from Fig. 2.2. A shock curve with fixed $u_{-}$and $v_{-}$corresponds to a vertical slice of $\mathscr{W}_{\text {base }}$. Such a slice can be parameterized by $v_{+}$, taking $s= \pm \sqrt{\Psi\left(v_{-}, v_{+}\right)}$and $\bar{u}=$ $u_{-}-\frac{1}{2} s\left(v_{+}-v_{-}\right)$. The shock curve typically has two disconnected branches, but the branches connect when $v_{-}=\alpha$ or $v_{-}=\beta$, forming crosses at the points $\left(v_{-}, v_{+}, s\right)=(\alpha, M, 0)$ and $\left(v_{-}, v_{+}, s\right)=(\beta, m, 0)$ in $\mathscr{W}_{\text {base }}$. Here $\alpha$ and $\beta$ demarcate the spinodal region: a chord of the graph of $p$ is horizontal if drawn between $\alpha$ and $M$ or between $m$ and $\beta$. Near the cross points, $\mathscr{W}_{\text {base }}$ has the form of a saddle with respect to the coordinate $v_{-}$; slicing $\mathscr{W}_{\text {base }}$ through the saddle point gives the cross structure.

2. For quadratic models, using Eq. (6.2) to eliminate $\bar{U}$ from $\mathscr{F}(\bar{U}, R, \Omega, s)=0$ yields $n$ linear equations for $R$ and $s$ with coefficients that depend upon $\Omega$. Consequently there are $n-2$ solvability constraints on $\Omega$, and $R$ and $s$ can be expressed in terms of points $\Omega$ satisfying them. (See refs. $[24,23]$ for the case $n=2$.)

A typical fixed- $U_{-}$shock curve in $\mathscr{W}$ is depicted schematically in Fig. 6.1 in terms of certain global coordinates $R, \kappa$, and $\Omega$ (cf. Fig. 3.2). The shock curve intersects $\mathscr{C}$ at two points, $L_{1}$ and $L_{2}$, which correspond to zero-strength shock waves with $U_{+}=U_{-}$and $s=\lambda_{i}(\bar{U})$ for $i=1,2$. There are three shock branches, the two local branches $A_{1} L_{1} A_{2} A_{3}$ and $L_{2} B$, and the detached branch through the point 
$C$. (The two copies of the point $A_{2}$ are to be identified, since they correspond to antipodal points.) Plotting the right states $U_{+}$along the shock curve yields the diagram at the bottom of the figure.

3. For KKIT models with $n=2$, a shock curve with fixed left state $U_{-} \neq 0$ is the union of two parts: the contact curve comprising points with $U_{+}$on the level curve of $\Phi$ containing $U_{-}$, i.e., $\Phi\left(U_{+}\right)=\Phi\left(U_{-}\right)$; and the scalar shock curve of points with $U_{+}$on the line through the origin that contains $U_{-}$. The shock foliation is singular at points with $U_{-}=0$, and it is singular everywhere if $n \geqq 3$, since level sets of $\Phi$ have codimension 1 .

As in the case of rarefaction curves, it proves useful to regard shock curves as integral curves of line fields. Notice that the $n$ component equations of $d U_{-}=0$ define a line field on the $(n+1)$-dimensional manifold $\mathscr{W}$. Furthermore, a fixed- $U_{-}$ shock curve in $\mathscr{W}$ is an integral curve of this line field, since $U_{-}$is constant along the shock curve. Similarly, fixed- $U_{+}$shock curves are integral curves of the line field $d U_{+}=0$.

Definition 6.2. The (possibly singular) line field on $\mathscr{W}$ defined by the equations $d U_{-}=0$ is called the fixed- $U_{-}$shock line field. Similarly, the fixed- $U_{+}$shock line field is defined by $d U_{+}=0$.

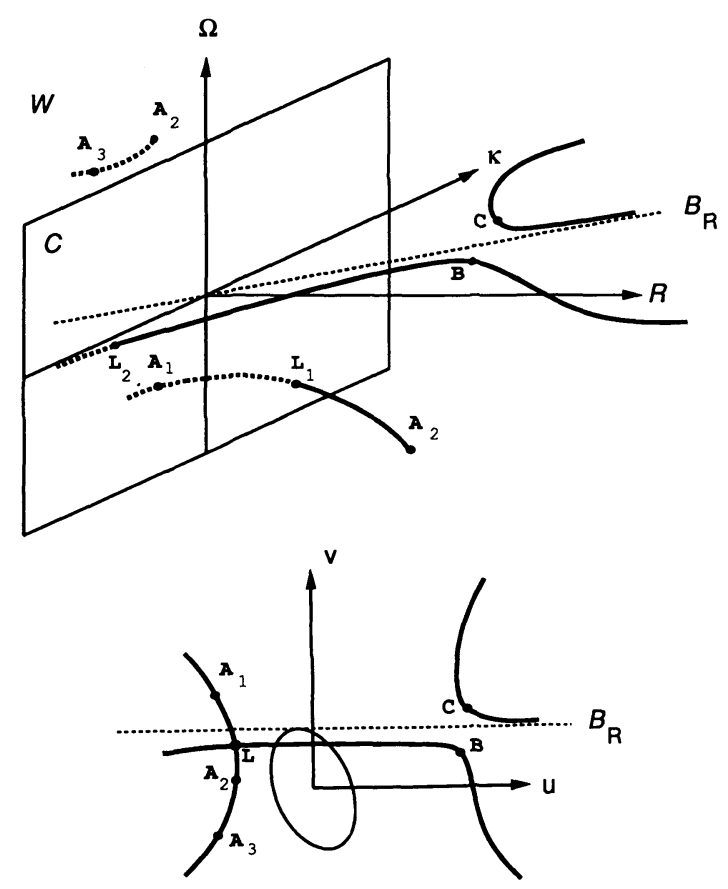

Fig. 6.1. A typical fixed- $U_{-}$shock curve for a quadratic model, drawn in terms of global coordinates $R, \kappa$, and $\Omega$ for $\mathscr{W}$. The shock curve intersects $\mathscr{C}$ at two points, labeled $L_{1}$ and $L_{2}$. The projections into the $(u, v)$-plane of the $U_{+}$states along the shock curve are shown below. The ellipse is the boundary of the elliptic region, which is the projection of the coincidence locus 
The occurrence of singularities in the shock line field corresponds to bifurcation of shock curves, as we now describe. For simplicity, the ensuing discussion will focus on shock curves with fixed left states. By the implicit function theorem, the points $(U, s)$ that satisfy Eq. (6.1) form a curve in a neighborhood of any solution for which the derivative

$$
-\left[U-U_{0}\right] d s+\left[-s+F^{\prime}(U)\right] d U
$$

has full rank. As already discussed, there is a singularity when $U=U_{0}$ and $s$ is an eigenvalue of $F^{\prime}\left(U_{0}\right)$. Because of this primary bifurcation, the shock curve has several branches emanating from $U_{0}$; away from eigenvalue coincidence, there is one for each real eigenvalue of $F^{\prime}\left(U_{0}\right)$. The primary bifurcation is removed by the blowingup process. (This is main purpose for blowing up.)

For nontrivial shock waves, $U \neq U_{0}$, the derivative has full rank unless: (a) $s$ is an eigenvalue of $F^{\prime}(U)$; and (b) $l\left(U-U_{0}\right)=0$ for some corresponding left eigenvector $l$ (which satisfies $l F^{\prime}(U)=s l$ ). Expressed differently, conditions (a) and (b) mean that the matrix $\left(U-U_{0},-s+F^{\prime}(U)\right)$ has rank less than $n$. This situation was identified by Wendroff [60] and is called secondary bifurcation. In the simplest cases, a shock curve has either a cross structure or an isolated point at a secondary bifurcation point; this follows from an analysis using bifurcation theory [18]. The geometric significance of secondary bifurcation is as singularities of the shock line field.

Theorem 6.3. A point of $\mathscr{W} \backslash \mathscr{C}$ is a singularity of the shock line field if and only if it is a secondary bifurcation point. A point of $\mathscr{C}$ is a shock singularity if and only if it is a singularity of the rarefaction line field.

Proof. Away from $\mathscr{C}$, we use the coordinates $\left(U_{-}, U_{+}, s\right)$ for $\mathscr{P} *$. A tangent vector $\left(\dot{U}_{-}, \dot{U}_{+}, \dot{S}\right)$ belongs to the line field $d U_{-}=0$ when $\dot{U}_{-}=0$; it is also tangent to $\mathscr{W}$ provided that

$$
-\left[U_{+}-U_{-}\right] \dot{s}+\left[-s+F^{\prime}\left(U_{+}\right)\right] \dot{U}_{+}=0,
$$

as seen from Eq. (2.15). Therefore the shock line field has singularities precisely when the linear system (6.5) has rank less than $n$, i.e., at secondary bifurcation points.

Evaluated at $R=0, d U_{-}=d \bar{U}-\frac{1}{2} r(\Omega) d R$. Thus a tangent vector $(\dot{\bar{U}}, \dot{R}, \dot{\Omega}, s)$ belongs to the shock line field at a point of $\mathscr{C}$ if and only if $\dot{\bar{U}}=\frac{1}{2} r(\Omega) \dot{R}$. Because $\mathscr{F}_{R}=0$ when $R=0$ by Lemma 2.5 , this vector must satisfy

$$
\frac{1}{2} \mathscr{F}_{\bar{U}} r(\Omega) \dot{R}+\mathscr{F}_{\Omega} \dot{\Omega}+\mathscr{F}_{s} \dot{s}=0
$$

to be tangent to $\mathscr{W}$. Hence shock singularities occur when $\left(\frac{1}{2} \mathscr{F}_{\bar{U}} r(\Omega), \mathscr{F}_{\Omega}, \mathscr{F}_{s}\right)$ is rank deficient. According to Proposition 4.2, this happens at singularities of the rarefaction line field.

Thus a singularity of the shock line field corresponds to bifurcation of the shock curve at the right state. This suggests a definition.

Definition 6.4. The right secondary bifurcation locus $\mathscr{B}_{R}$ is the set of points in $\mathscr{W}$ where the shock line field $d U_{-}=0$ is singular. Similarly, the left secondary bifurcation locus $\mathscr{B}_{L}$ comprises singularities of the shock line field $d U_{+}=0$.

Corollary 6.5. The fixed-U _ shock curves constitute a one-dimensional foliation of $\mathscr{W} \backslash \mathscr{B}_{R}$, and the fixed- $U_{+}$shock curves foliate $\mathscr{W} \backslash \mathscr{B}_{L}$. 
Corollary 6.6. The locus of rarefaction singularities is $\mathscr{B}_{R} \cap \mathscr{C}=\mathscr{B}_{0}=\mathscr{B}_{L} \cap \mathscr{C}$.

Secondary bifurcation requires that an $n \times(n+1)$ matrix be rank deficient, and thus represents two conditions on points of $\mathscr{W}$. Therefore, if certain nondegeneracy conditions holds, $\mathscr{B}_{R}$ has dimension $n-1$, and it intersects $\mathscr{C}$ transversally. In determining the smoothness of $\mathscr{B}_{R}$ at points of $\mathscr{C}$, the following formulae are useful.

Proposition 6.7. The points of the secondary bifurcation locus $\mathscr{B}_{R}$ are the points where the matrix $\left(\mathscr{F}_{R}+\frac{1}{2} \mathscr{F}_{\bar{U}} r(\Omega), \mathscr{F}_{\Omega}+\frac{1}{2} R \mathscr{F}_{\bar{U}} r^{\prime}(\Omega), \mathscr{F}_{s}\right)$ is rank deficient. Similarly, the matrix $\left(\mathscr{F}_{R}-\frac{1}{2} \mathscr{F}_{\bar{U}} r(\Omega), \mathscr{F}_{\Omega}-\frac{1}{2} R \mathscr{F}_{\bar{U}} r^{\prime}(\Omega), \mathscr{F}_{s}\right)$ is rank deficient at points of $\mathscr{B}_{L}$.

Proof. A tangent vector $(\dot{\bar{U}}, \dot{R}, \dot{\Omega}, \dot{s})$ belongs to the shock line field $d U_{-}=0$ at a point of $\mathscr{W}$ if and only if $\dot{\bar{U}}=\frac{1}{2} r(\Omega) \dot{R}+\frac{1}{2} R r^{\prime}(\Omega) \dot{\Omega}$. Combining this with the condition for tangency to $\mathscr{W}$ yields

$$
\left[\mathscr{F}_{R}+\frac{1}{2} \mathscr{F}_{\bar{U}} r(\Omega)\right] \dot{R}+\left[\mathscr{F}_{\Omega}+\frac{1}{2} R \mathscr{F}_{\bar{U}} r^{\prime}(\Omega)\right] \dot{\Omega}+\mathscr{F}_{s} \dot{s}=0 .
$$

Since $\dot{\bar{U}}$ is determined from $\dot{R}$ and $\dot{\Omega}$, singularities of the line field occur if and only if the matrix of Eq. (6.7) has rank less than $n$.

\section{Examples.}

1. The bifurcation loci are empty for a van der Waals gas if $T>1$. For $T<1$, the bifurcation locus $\mathscr{B}_{L}$ consists of two lines parameterized by $\bar{u}$; these lines correspond to the cross points $\left(v_{-}, v_{+}\right)=(\alpha, M)$ and $\left(v_{-}, v_{+}\right)=(\beta, m)$ described above. Similarly,

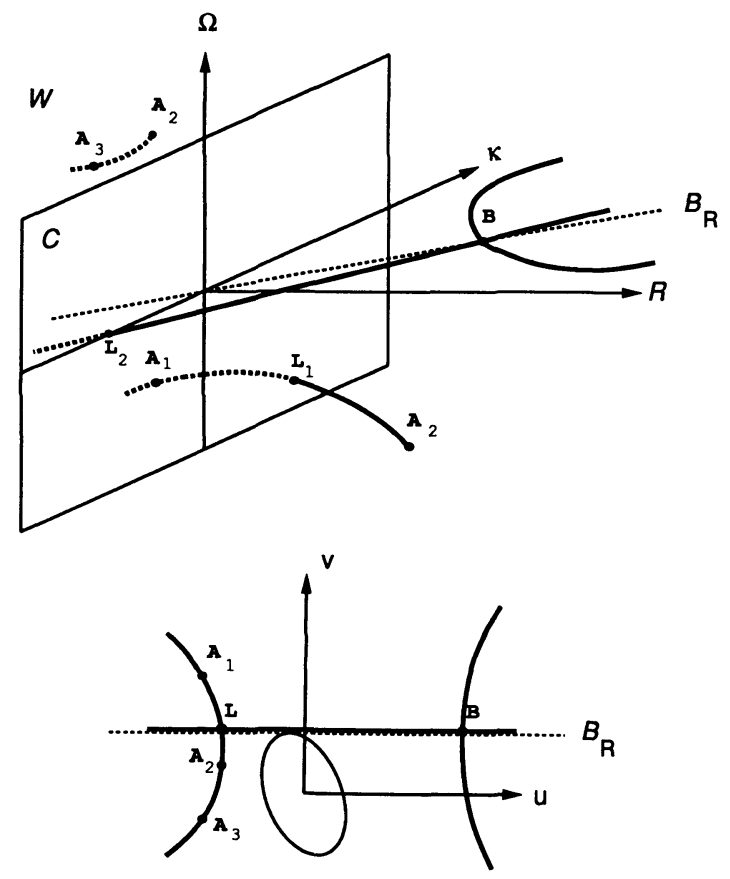

Fig. 6.2. The shock curve through the point $B$ on the secondary bifurcation locus $\mathscr{B}_{R}$. This shock curve has a cross structure at $B$; under a small perturbation, it bifurcates into the shock curve shown in Fig. 6.1, with point $B$ splitting into points $B$ and $C$. The projections of the $U_{+}$states along the shock curve are shown below. 
the bifurcation locus $\mathscr{B}_{R}$ corresponds to the cross points $\left(v_{-}, v_{+}\right)=(M, \alpha)$ and $\left(v_{-}, v_{+}\right)=(m, \beta)$.

2. For quadratic models with $n=2$, the condition of Proposition 6.7 reduces to a homogeneous cubic polynomial equation in $\cos \Omega$ and $\sin \Omega$, which has roots $\Omega$ called the asymptotic angles, together with three linear equations relating $\bar{U}, R$, and $s$. Thus the secondary bifurcation locus consists of one, two, or three lines [23]. At each point along the bifurcation lines, the shock foliation has a saddle point singularity, so that the shock curve has a cross structure. The shock curve through a secondary bifurcation point $B \in \mathscr{B}_{R}$ is depicted in Fig. 6.2.

3. For KKIT models with $n=2$, a shock point is in $\mathscr{B}_{R}$ if and only if either: it lies on $\mathscr{W}_{\text {contact }}$ and $\Phi^{\prime}\left(U_{+}\right)=0$ (so that the level curve of $\Phi$ has a singularity at $U_{+}$); or it belongs to both $\mathscr{W}_{\text {contact }}$ and $\mathscr{W}_{\text {ruled }}$, i.e., it is a contact discontinuity as well as a shock wave for the associated scalar conservation law.

Remark. At a secondary bifurcation, in the least degenerate instances, a shock curve has either a cross structure or an isolated point. The nature of the singular point for the shock foliation is determined by unfolding these singularities: when the shock curve has a cross structure, the foliation has a saddle point; when the shock curve has an isolated point, the foliation has a center.

The understand the relationship between shock curves and rarefaction curves, consider how the shock foliation intersects $\mathscr{C}$. According to Eq. (6.6), the vector witth components

$$
\begin{aligned}
\dot{\bar{U}} & =\operatorname{det}\left(\mathscr{F}_{\Omega}, \mathscr{F}_{s}\right) r(\Omega), \\
\dot{R} & =2 \operatorname{det}\left(\mathscr{F}_{\Omega}, \mathscr{F}_{s}\right), \\
\left(\begin{array}{c}
\dot{\Omega} \\
\dot{s}
\end{array}\right) & =-\operatorname{adj}\left(\mathscr{F}_{\Omega}, \mathscr{F}_{s}\right) \mathscr{F}_{\bar{U}} r(\Omega),
\end{aligned}
$$

is tangent to the shock foliation at a point of $\mathscr{C} \backslash \mathscr{B}_{0}$. Therefore the vector of Eq. (4.4), which is tangent to the rarefaction foliation, has the same components except that $\dot{R}=0$. At a point $p \in \mathscr{P}_{0}^{*}$, the projection from $T_{p} \mathscr{P}^{*}$ to $T_{p} \mathscr{P}_{0}^{*}$ that sets the $R$-component to zero is defined invariantly, as shown in the Appendix. For this reason the rarefaction line field on $\mathscr{C}$ is induced naturally by the shock line field on $\mathscr{W}$.

Shock curves in $\mathscr{U}$ are obtained from shock curves in $\mathscr{W}$ through the projection map $(\bar{U}, R, \Omega, s) \mapsto U_{+}$. This projection is one-to-one away from $U=U_{0}$ : if both $\left(U_{0}, U, s_{1}\right)$ and $\left(U_{0}, U, s_{2}\right)$ satisfy Eq. (6.1) with $U \neq U_{0}$, then $s_{2}=s_{1}$. As the next result describes, the projection is an immersion except on $\mathscr{E} \cup \mathscr{B}_{R}$.

Proposition 6.8. The $U_{+}$map, restricted to a fixed- $U_{-}$shock curve, is singular at $p \in \mathscr{W} \backslash \mathscr{B}_{R}$ if and only if $p \in \mathscr{E}$. Moreover the shock curve through a fold point of $\mathscr{E} \backslash \mathscr{B}_{0}$ is transverse to $\mathscr{E}$, and its $U_{+}$projection has a cusp.

Proof. Away from $\mathscr{C}$, a tangent vector $\left(\dot{U}_{-}, \dot{U}_{+}, \dot{s}\right) \neq 0$ to the shoçk curve satisfies Eq. (6.5) and $\dot{U}_{-}=0$, which implies that $\dot{U}_{+} \neq 0$. On $\mathscr{C}, \dot{U}_{+}=2 \dot{\bar{U}}$, and according to Eqs. (6.8), this vector vanishes if and only if $\operatorname{det}\left(\mathscr{F}_{\Omega}, \mathscr{F}_{s}\right)=0$. In this case $\dot{R}=0$, i.e., the shock curve is tangent to the rarefaction curve, and the result follows from Prop. 4.5. 


\section{The Sonic Locus $\mathscr{S}$}

Not all solutions of the Rankine-Hugoniot condition (1.3) represent physical shock waves. The conservation laws (1.1) usually arise from a parabolic equation of the form

$$
U_{t}+F(U)_{x}=\varepsilon\left(D(U) U_{x}\right)_{x}
$$

in the singular limit as $\varepsilon \rightarrow 0$; here $D=D(U)$ is called the viscosity matrix. A physical shock wave is a solution of the Rankine-Hugoniot condition that is the limit, as $\varepsilon \rightarrow 0$, of a stable solution of Eq. (7.1).

Determining whether a shock wave is physical is difficult, and simple criteria are useful when solving Reimann problems. The Lax criterion is typical. Define a shock wave to be a Lax shock of the $i^{\text {th }}$ family [33] if

$$
\begin{gathered}
\lambda_{i}\left(U_{+}\right)<s<\lambda_{i}\left(U_{-}\right), \\
\lambda_{i-1}\left(U_{-}\right)<s<\lambda_{i+1}\left(U_{+}\right) .
\end{gathered}
$$

It is known that for strictly hyperbolic and genuinely nonlinear systems of conservation laws, a weak Lax shock wave is physical (for the viscosity matrix $D(U) \equiv I$ ).

Proving this involves two steps: (1) showing that a weak Lax wave admits a viscous profile [12,7]; and (2) showing that the viscous profile is stable [39]. A viscous profile of the shock wave $\left(U_{-}, U_{+}, s\right)$ is a traveling-wave solution $U(x, t)=$ $\tilde{U}((x-s t) / \varepsilon)$ of Eq. (7.1) for which $\tilde{U}(\xi) \rightarrow U_{ \pm}$as $\xi \rightarrow \pm \infty$; such a solution tends (in $\left.L_{\text {loc }}^{1}\right)$ to the shock wave solution as $\varepsilon \rightarrow 0$. To be a viscous profile, $\widetilde{U}$ must be an orbit of the dynamical system

$$
D(\tilde{U}) \tilde{U}^{\prime}=-s\left[\tilde{U}-U_{-}\right]+F(\tilde{U})-F\left(U_{-}\right)
$$

that connects the critical points $U_{-}$and $U_{+}$. When $D(U) \equiv I$, the Lax criterion means that the dimensions of the unstable manifold of $U_{-}$and the stable manifold of $U_{+}$add to $n+1$, which essentially guarantees existence and stability of a connecting orbit if $U_{-}$and $U_{+}$are close.

For strong shock waves, the Lax criterion is not adequate. Even for scalar conservation laws $(n=1)$ there are Lax shock waves that do not admit viscous profiles; this phenomenon is important for systems, too [56, 19]. Furthermore, transitional and overcompressive shock waves (which do not satisfy the Lax criterion) can enter the solution of the Riemann problem $[8,55,62,4,23,51,49,13,40]$. For simplicity, however, we study only the Lax criterion in the present work; this is a necessary first step in understanding more realistic admissibility criteria. Moreover, the Lax criterion, viewed in the context of the fundamental wave manifold, illuminates the relationship between different admissibility criteria. Shock waves satisfying inequalities (7.2) correspond to open regions in $\mathscr{W}$, the boundaries of which are parts of hypersurfaces that we define in this section. Similarly, other criteria also correspond to regions of $\mathscr{W}$, and the admissibility boundaries overlap with these hypersurfaces.

Remarks. The fundamental wave manifold offers a framework for studying the dynamical systems associated with shock waves [6, 5]. Indeed, Eq. (7.3) defines a map from $\mathscr{W}$ to the space $\mathscr{X}(\mathscr{U})$ of vector fields on $\mathscr{U}$. Thus $\mathscr{W}$ parameterizes a family of dynamical systems, which depends on the viscosity matrix $D$. 
Consider a shock curve in $\mathscr{W}$ with fixed left state. The portions of this curve consisting of Lax shock waves are delimited by points at which either $s=\lambda_{i}\left(U_{-}\right)$or $s=\lambda_{i}\left(U_{+}\right)$. By analogy with gas dynamics, such a shock wave is called sonic (on the left or right, respectively). Thus the boundaries of the Lax admissibility regions consist of sonic shock waves. Bethe [2] and Wendroff [60] related the sonic condition to the behavior of $s$ along the shock curve.

Proposition 7.1. Consider a point $U \neq U_{0}$ on the shock curve in $\mathscr{U}$ through $U_{0}$; suppose that it is not a secondary bifurcation point. Then the shock speed $s$ is an eigenvalue of $F^{\prime}(U)$ if and only if the graph of $s$ along the shock curve has a stationary point at $U$.

Moreover, at such a stationary point, the graph of s has vanishing second derivative if and only if $l F^{\prime \prime}(U) r^{2}=0$, where $l$ and $r$ nonzero left and right null vectors of the matrix $-s+F^{\prime}(U)$, respectively.

Proof. Since $U$ is not a secondary bifurcation point, the shock curve through $U_{0}$ is a parameterized curve in a neighborhood of $U$. Differentiating Eq. (6.1) with respect to the parameter shows that

$$
-\left[U-U_{0}\right] \dot{s}+\left[-s+F^{\prime}(U)\right] \dot{U}=0,
$$

where $(\dot{U}, \dot{s}) \neq 0$. If $\dot{s}=0$, then $\dot{U} \neq 0$, so that $s$ is an eigenvalue of $F^{\prime}(U)$. Conversely, suppose that $s$ is an eigenvalue of $F^{\prime}(U)$. Then Eq. (7.4), multiplied on the left by a corresponding left eigenvector $l$, implies that $l\left[U-U_{0}\right] \dot{s}=0$, where, by assumption, $l\left[U-U_{0}\right] \neq 0$.

Differentiating Eq. (7.4) and evaluating at a point where $\dot{s}=0$ shows that $\left[U-U_{0}\right] \ddot{s}=F^{\prime \prime}(U) \dot{U}^{2}+\left[-s+F^{\prime}(U)\right] \ddot{U}$. Thus $\ddot{s}=0$ if and only if $l F^{\prime \prime}(U) r^{2}=0$, with $r:=\dot{U}$.

The Bethe-Wendroff Theorem can be rephrased in terms of the shock foliation of the manifold $\mathscr{W}$. In this context, stationary points for $s$, regarded as a function along a shock curve, occur where the shock line field lies in the hyperplane $d s=0$.

Lemma 7.2. At a point $p:=(\bar{U}, R, \Omega, s) \in \mathscr{W}$, there is a nonzero vector contained in both the shock line field $d U_{-}=0$ and the tangent hyperplane $d s=0$ if and only if the matrix $B_{+}(\bar{U}, R, \Omega, s):=\left(\mathscr{F}_{R}+\frac{1}{2} \mathscr{F}_{\bar{U}} r(\Omega), \mathscr{F}_{\Omega}+\frac{1}{2} R \mathscr{F}_{\bar{U}} r^{\prime}(\Omega)\right)$ is singular.

Proof. Let $(\dot{\bar{U}}, \dot{R}, \dot{\Omega}, \dot{s}) \neq 0$ belong to the shock line field, so that we have $\dot{\bar{U}}=\frac{1}{2} r(\Omega) \dot{R}+\frac{1}{2} R^{\prime} r(\Omega) \dot{\Omega}$. According to Eq. $(6.7), \dot{s}=0$ only if $(\dot{R}, \dot{\Omega})$ is a right null vector of $B_{+}(\bar{U}, R, \Omega, s)$. Conversely, if $(\dot{R}, \dot{\Omega}) \neq 0$ is a null vector of $B_{+}$and $\dot{\bar{U}}:=\frac{1}{2} r(\Omega) \dot{R}+\frac{1}{2} R r^{\prime}(\Omega) \dot{\Omega}$, then $(\dot{\bar{U}}, \dot{R}, \dot{\Omega}, 0) \neq 0$ is tangent to $\mathscr{W}$ and belongs to both the shock line field and the hyperplane $d s=0$.

Theorem 7.3. At a point $\left(U_{-}, U_{+}, s\right) \in \mathscr{W} \backslash \mathscr{B}_{R}$ with $U_{+} \neq U_{-}$, the shock line field $d U_{-}=0$ is contained in the tangent hyperplane $d s=0$ if and only if $s$ is an eigenvalue of $F^{\prime}\left(U_{+}\right)$. On $\mathscr{C} \backslash \mathscr{B}_{0}$, points where the shock line field lies in $d s=0$ are points of inflection.

Proof. When $p \notin \mathscr{C}$, we can use the coordinates $\left(U_{-}, U_{+} . s\right)$ for $\mathscr{P}^{*}$. If $\left(\dot{U}_{-}, \dot{U}_{+}, \dot{s}\right)$ belongs to the shock line field, then $\dot{U}_{-}=0$ and Eq. (6.5) holds. Thus $\dot{s}=0$ if and only if $\dot{U}_{+}$is an eigenvector of $F^{\prime}\left(U_{+}\right)$with eigenvalue $s$. When $p \in \mathscr{C}, R=0$ and 
$\mathscr{F}_{R}=0$, so that $\mathscr{B}_{+}(\bar{U}, 0, \Omega, s)=\left(\frac{1}{2} \mathscr{F}_{\bar{U}} r(\Omega), \mathscr{F}_{\Omega}\right)$; this matrix is singular if and only if $p \in \mathscr{I}$.

Definition 7.4. The right sonic locus $\mathscr{S}_{R}$ consists of points in $\mathscr{W}$ where there is a nonzero vector contained in both the shock line field $d U_{-}=0$ and the tangent hyperplane $d s=0$, i.e., the matrix $B_{+}(\bar{U}, R, \Omega, s)$ is singular. The left sonic locus $\mathscr{S}_{L}$ is defined similarly for the shock line field $d U_{+}=0$; it comprises points where the matrix $B_{-}(\bar{U}, R, \Omega, s):=\left(\mathscr{F}_{R}-\frac{1}{2} \mathscr{F}_{\bar{U}} r(\Omega), \mathscr{F}_{\Omega}-\frac{1}{2} R \mathscr{F}_{\bar{U}} r^{\prime}(\Omega)\right)$ is singular.

In working with $B_{ \pm}$, the following formulae are useful:

$$
R \cdot B_{ \pm}(\bar{U}, R, \Omega, s)=\left[-s+F^{\prime}\left(U_{ \pm}\right)\right]\left(r(\Omega), R r^{\prime}(\Omega)\right)
$$

for any $(\bar{U}, R, \Omega, s) \in \mathscr{W}$, as is verified by a straightforward calculation using Eq. (2.15). In particular, near a point with $R \neq 0$, the sonic locus $\mathscr{S}_{R}$ is defined by the equation $\operatorname{det}\left[-s+F^{\prime}\left(U_{+}\right)\right]=0$.

The sonic loci $\mathscr{S}_{L}$ and $\mathscr{S}_{R}$ are $n$-dimensional submanifolds of $\mathscr{W}$ when certain nondegeneracy conditions hold. For instance, $\mathscr{S}_{R}$ is smooth if zero is a regular value of det $B_{+}$on $\mathscr{W}$. While such a condition is expected to hold for generic flux functions $F$, there are cases when it is violated. For example, $\mathrm{Xu}$ [63] has observed singularities of $\mathscr{S}_{R}$ in a model of three-phase immiscible flow. In this model, there are shock waves $\left(U_{-}, U_{+}, s\right)$ with $U_{+} \neq U_{-}$such that $s$ is a multiple eigenvalue of $F^{\prime}\left(U_{+}\right)$with geometric multiplicity greater than 1 , i.e., $U_{+}$is a (projected) umbilic point. The right sonic locus $\mathscr{S}_{R}$ is singular at such a point because $-s+F^{\prime}\left(U_{+}\right)$ has rank less than $n-1$. For simplicity of exposition, however, the following assumption will be made.

Assumption 7.5. Zero is a regular value of both $\operatorname{det} B_{+}$and $\operatorname{det} B_{-}$on $\mathscr{W}$.

The sonic loci are related to other loci, as follows immediately from their definitions.

Corollary 7.6. The secondary bifurcation loci are contained in the sonic loci: $\mathscr{B}_{R} \subseteq \mathscr{S}_{R}$ and $\mathscr{B}_{L} \subseteq \mathscr{S}_{L}$. Furthermore, the inflection locus is $\mathscr{S}_{L} \cap \mathscr{C}=\mathscr{I}=\mathscr{S}_{R} \cap \mathscr{C}$.

According to Proposition 7.1, when a shock curve intersects $\mathscr{S}_{R} \backslash \mathscr{B}_{R}$, the graph of $s$ has an extremum unless genuine nonlinearity fails at the state $U$. The latter situation was termed hysteresis by Shearer and Schaeffer [54]. As the following result shows, hysteresis occurs when the shock curve is tangent to $\mathscr{S}_{R}$.

Proposition 7.7. Suppose that $p:=\left(U_{-}, U_{+}, s\right) \in \mathscr{S}_{R} \backslash \mathscr{B}_{R}$ with $U_{+} \neq U_{-}$. Consider the graph of $s$ along the fixed- $U_{-}$shock curve through $p$. This graph has vanishing second derivative at $p$ if and only if the shock curve is tangent to $\mathscr{S}_{R}$ at $p$.

Proof. Since $p \notin \mathscr{C}$, we can use the coordinates $\left(U_{-}, U_{+}, s\right)$ for $\mathscr{P} *$ in a sufficiently small neighborhood of $p$. The portion of $\mathscr{S}_{R}$ contained in this neighborhood is the subset of $\mathscr{W}$ where the matrix $-s+F^{\prime}\left(U_{+}\right)$is singular. Let $\left(0, \dot{U}_{+}, 0\right) \neq 0$ be tangent to the fixed- $U_{-}$shock curve at $p$; here $\dot{U}_{+}$must satisfy $\left[-s+F^{\prime}\left(U_{+}\right)\right] \dot{U}_{+}=0$ for this vector to be tangent to $\mathscr{W}$. To determine when this vector is tangent to $\mathscr{S}_{R}$, we invoke Lemma 5.5.

Let $l$ and $r:=U_{+}$be nonzero left and right null vectors of $-s+F^{\prime}\left(U_{+}\right)$, respectively. The derivative of $-s+F^{\prime}\left(U_{+}\right)$, applied to the shock tangent, is 
$F^{\prime \prime}\left(U_{+}\right) U_{+}$. Therefore the shock curve is tangent to $S_{R}$ if and only if $l F^{\prime \prime}\left(U_{+}\right) r^{2}=0$. The result now follows from Proposition 7.1.

Definition 7.8. The right hysteresis locus $\mathscr{H}_{R}$ consists of points in $\mathscr{S}_{R}$ such that $l \dot{B}_{+} r=0$, where $l$ and $r:=(\dot{R}, \dot{\Omega})$ are nonzero left and right null vectors of $B_{+}$, respectively, and $\dot{B}_{+}$denotes $B_{+}^{\prime}$ applied to the vector $(\dot{\bar{U}}, \dot{R}, \dot{\Omega}, 0)$ with $\dot{\bar{U}}:=\frac{1}{2} r(\Omega) \dot{R}+\frac{1}{2} R r^{\prime}(\Omega) \dot{\Omega}$. The left hysteresis locus $\mathscr{H}_{L}$ is defined analogously for the shock line field $d U_{+}=0$.

Corollary 7.9. Suppose that $p \in \mathscr{S}_{R} \backslash \mathscr{B}_{R}$. Then the fixed-U - shock curve through $p$ is transverse to $\mathscr{S}_{R}$ at $p$ if and only if $p \notin \mathscr{H}_{R}$.

According to Propositions 7.1 and 7.7, a point of $\mathscr{S}_{R} \backslash \mathscr{C}$ belongs to $\mathscr{H}_{R}$ if and only if $l F^{\prime \prime}\left(U_{+}\right) \dot{U}_{+}^{2}=0$. For points of $\mathscr{C}$, the condition for hysteresis is related to the condition for tangency of a rarefaction curve to the inflection locus, as we now demonstrate.

Lemma 7.10. Suppose that $p:=(\bar{U}, 0, \Omega, s) \in \mathscr{C}$. Let $l$ and $r:=(\dot{R}, \dot{\Omega})$ be nonzero left and right null vectors of $B_{+}(\bar{U}, 0, \Omega, s)$, respectively, and let $\dot{B}_{+}$denote $B_{+}^{\prime}$ evaluated at $p$ and applied to the vector $(\bar{u}, \rho, \omega, \sigma)$. Then

$$
l \dot{B}_{+} r=\frac{1}{12} \rho \dot{R} l F^{\prime \prime \prime}(\bar{U}) r(\Omega)^{3}+\frac{1}{2} \rho l F^{\prime \prime}(\bar{U}) \cdot\left(r(\Omega) \otimes r^{\prime}(\Omega) \dot{\Omega}\right)+l \dot{B}_{0} r,
$$

where $B_{0}$ is the matrix of Definition 5.4, and $\dot{B}_{0}$ denotes $B_{0}^{\prime}$ applied to the vector $(\bar{u}, \omega, \sigma)$.

Proof. We have that $B_{+}(\bar{U}, 0, \Omega, s)=B_{0}(\bar{U}, \Omega, s)$. Furthermore, partial derivatives of $\mathscr{F}$ that have odd order with respect to $R$ vanish at $R=0$ by Lemma 2.5. On $R=0$, therefore, $\dot{B}_{+}$simplifies to be $\dot{B}_{+}=\rho\left(\mathscr{F}_{R R}, \frac{1}{2} \mathscr{F}_{\bar{U}} r^{\prime}(\Omega)\right)+\dot{B}_{0}$. From the definition (2.7) of $A$ we find that $\mathscr{F}_{R R}=\frac{1}{12} F^{\prime \prime \prime}(\bar{U}) r(\Omega)^{3}$, so that Eq. (7.6) follows.

Proposition 7.11. A point $p$ belongs to the exceptional inflection locus $\mathscr{H}_{0}$ if and only if either $p \in \mathscr{H}_{R} \cap \mathscr{C}$ or $\mathscr{S}_{R}$ is not transverse to $\mathscr{C}$ at $p$. Also, $\mathscr{H}_{L} \cap \mathscr{C}=\mathscr{H}_{R} \cap \mathscr{C}$.

Proof. We adopt the notation of the lemma. By definition, $p \in \mathscr{H}_{0}$ if and only if either (a) $p$ is not a regular point of $\operatorname{det} B_{0}$ or (b) $l \dot{B}_{0} r=0$ where $\dot{B}_{0}$ denotes $B_{0}^{\prime}$ applied to $(\bar{u}, \omega, \sigma):=\left(\frac{1}{2} \dot{R} r(\Omega), \dot{\Omega}, 0\right)$. Given Assumption 7.5, Eq. (7.6) implies that (a) occurs if and only if all vectors tangent to $\mathscr{S}_{R}$ have $\dot{R}=0$. Thus condition (a) holds if and only if $\mathscr{S}_{R}$ is not transverse to $\mathscr{C}$ at $p$. Furthermore, the same equation shows that when $(\bar{u}, \rho, \omega, \sigma):=\left(\frac{1}{2} \dot{R} r(\Omega), \dot{R}, \dot{\Omega}, 0\right), l \dot{B}_{+} r=\frac{3}{4} l \dot{B}_{0} r$; see Eq. (5.4). Consequently, (b) holds if and only if $p \in \mathscr{H}_{R} \cap \mathscr{C}$. From the form of condition (b), it is evident that $\mathscr{H}_{L} \cap \mathscr{C}=\mathscr{H}_{R} \cap \mathscr{C}$.

Transversality between the fixed-U $\mathrm{U}_{-}$shock foliation and $\mathscr{S}_{R}$ is important for wave curve bifurcation. Similarly, it is useful to characterize when the other shock foliation, for which $U_{+}$is fixed, is transverse to $\mathscr{S}_{R}$.

Proposition 7.12. Suppose that $p:=\left(U_{-}, U_{+}, s\right) \in \mathscr{S}_{R} \backslash \mathscr{B}_{L}$ with $U_{+} \neq U_{-}$. Then the fixed- $U_{+}$shock curve through $p$ is tangent to $\mathscr{S}_{R}$ at $p$ if and only if the shock wave $\left(U_{-}, U_{+}, s\right)$ is doubly sonic, i.e., $s$ is an eigenvalue of the matrix $F^{\prime}\left(U_{-}\right)$as well as of $F^{\prime}\left(U_{+}\right)$.

Proof. Near $p \in \mathscr{C}$, we can use the coordinates $\left(U_{-}, U_{+}, s\right)$ for $\mathscr{P}^{*}$, and $\mathscr{S}_{R}$ is the subset of $\mathscr{W}$ where the matrix $-s+F^{\prime}\left(U_{+}\right)$is singular. Let the nonzero vector 
$\left(\dot{U}_{-}, 0, \dot{s}\right)$ be tangent to the fixed- $U_{+}$shock curve at $p$, so that $-\left[U_{+}-U_{-}\right] \dot{s}-[-s+$ $\left.F^{\prime}\left(U_{-}\right)\right] \dot{U}_{-}=0$. Since the derivative of $-s+F^{\prime}\left(U_{+}\right)$applied to this vector is $-\dot{s} I$, Lemma 5.5 and Assumption7.5 imply that transversality between the shock curve and $\mathscr{S}_{R}$ is equivalent to $\dot{s} \neq 0$. Consequently, the shock curve is tangent to $\mathscr{S}_{R}$ if and only if $s$ is an eigenvalue of $F^{\prime}\left(U_{-}\right)$.

Doubly sonic shock waves are points of $\mathscr{S}_{L} \cap \mathscr{S}_{R}$ with $U_{+} \neq U_{-}$. More generally, the intersection of the sonic loci consists of points of $\mathscr{W}$ for which $\operatorname{det} B_{-}=0$ and $\operatorname{det} B_{+}=0$. As seen by expanding these determinants, such points satisfy the equations

and

$$
\operatorname{det}\left(\mathscr{F}_{R}, \mathscr{F}_{\Omega}\right)+R \operatorname{det}\left(\frac{1}{2} \mathscr{F}_{\bar{U}} r(\Omega), \frac{1}{2} \mathscr{F}_{\bar{U}} r^{\prime}(\Omega)\right)=0
$$

$$
\operatorname{det}\left(\frac{1}{2} \mathscr{F}_{\bar{U}} r(\Omega), \mathscr{F}_{\Omega}\right)+R \operatorname{det}\left(\mathscr{F}_{R}, \frac{1}{2} \mathscr{F}_{\bar{U}} r^{\prime}(\Omega)\right)=0 .
$$

Notice that when $R=0$, Eq. (7.7) is satisfied because $\mathscr{F}_{R}=0$, while Eq. (7.8) is satisfied precisely on the inflection locus $\mathscr{I}$. In fact, $\mathscr{S}_{L} \cap \mathscr{S}_{R}$ is the union of $\mathscr{I}$ with a locus that contains the set of doubly sonic shock waves. An equation defining the latter is obtained by removing a factor of $R$ from Eq. (7.7).

Definition 7.13. The double sonic locus $\mathscr{D}$ consists of points in $\mathscr{W}$ such that

and

$$
\operatorname{det}\left(R^{-1} \mathscr{F}_{R}, \mathscr{F}_{\Omega}\right)+\operatorname{det}\left(\frac{1}{2} \mathscr{F}_{\bar{U}} r(\Omega), \frac{1}{2} \mathscr{F}_{\bar{U}} r^{\prime}(\Omega)\right)=0
$$

$$
\operatorname{det}\left(\frac{1}{2} \mathscr{F}_{\bar{U}} r(\Omega), \mathscr{F}_{\Omega}\right)+R^{2} \operatorname{det}\left(R^{-1} \mathscr{F}_{R}, \frac{1}{2} \mathscr{F}_{\bar{U}} r^{\prime}(\Omega)\right)=0
$$

Here $R^{-1} \mathscr{F}_{R}$ denotes the smooth extension of this function, as defined on $\mathscr{W} \backslash \mathscr{C}$, to all of $\mathscr{W}$ :

$$
R^{-1} \mathscr{F}_{R}(\bar{U}, R, \Omega, s):=\int_{-1 / 2}^{1 / 2} \int_{0}^{1} \alpha^{2} F^{\prime \prime \prime}(\bar{U}+\alpha \beta R r(\Omega)) r(\Omega)^{3} d \beta d \alpha .
$$

Corollary 7.14. The intersection of the sonic loci is $\mathscr{S}_{L} \cap \mathscr{S}_{R}=\mathscr{I} \cup \mathscr{D}$.

\section{Examples.}

0 . The relationships among the loci is elucidated by an example of a scalar conservation law. In the scalar case, $\mathscr{W}$ is simply the graph of the shock speed $s=\int_{-1 / 2}^{1 / 2} F^{\prime}(\bar{U}+\alpha R) d \alpha=\int_{0}^{1} F^{\prime}\left((1-\alpha) U_{-}+\alpha U_{+}\right) d \alpha$. Thus $U_{-}$and $U_{+}$serve as global coordinates, and the characteristic manifold is the diagonal $U_{+}=U_{-}$. The fixed- $U_{-}$shock foliation consists of vertical lines, and fixed- $U_{+}$shock curves are horizontal. Figure 7.1 shows $\mathscr{W}$ when the flux is a double well, $F(U)=\left(U^{2}-1\right)^{2}$. The sonic loci are ellipses that intersect $\mathscr{C}$ at $\mathscr{I}$, which comprises the two inflection points of $F$. Points of the double sonic locus correspond to shock waves between the minima \pm 1 of the flux.

1. As we have seen, the fundamental wave manifold for a van der Waals gas with $T<1$ is $\mathscr{W}=\mathscr{W}_{\text {base }} \times \mathbf{R}$, where $\mathscr{W}_{\text {base }}$ is a cylinder obtained by gluing two copies of Fig. 7.2 along the boundary of the black hole. Similarly, each distinguished submanifold is a submanifold of $\mathscr{W}_{\text {base }}$ crossed with $\mathbf{R}$. The left and right sonic loci $\mathscr{S}_{L}$ and $\mathscr{S}_{R}$ correspond to points where the level curves of $s$ in Fig. 2.2 have horizontal and vertical tangents, respectively. 


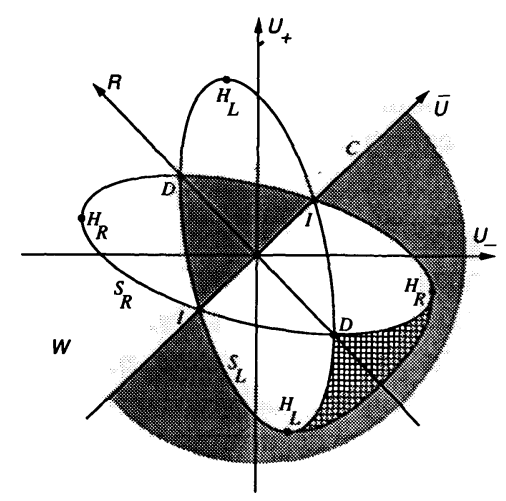

Fig. 7.1. The fundamental wave manifold $\mathscr{W}$ for the scalar model $U_{t}+\left[\left(U^{2}-1\right)^{2}\right]_{x}=0$. Shown here are the characteristic manifold $\mathscr{C}$, the sonic loci $\mathscr{S}_{L}$ and $\mathscr{S}_{R}$, the inflection locus $\mathscr{I}$, the double sonic locus $\mathscr{D}$, and the hysteresis loci $\mathscr{H}_{L}$ and $\mathscr{H}_{R}$. Shock waves in the shaded regions satisfy the Oleinik admissibility criterion; those in the cross-hatched region satisfy the Lax criterion but not the Oleinnik criterion

Recall that a fixed- $U_{-}$shock curve is parameterized by $v_{+}$, and that $s^{2}=\Psi\left(v_{-}, v_{+}\right)$. Therefore extrema of $s$ occur along a shock curve at points where $\partial \Psi / \partial v_{+}=0$, and $\mathscr{S}_{R}$ is defined by this equation. As follows from a simple calculation, this means that $s^{2}=-p^{\prime}\left(v_{+}\right)$on $\mathscr{S}_{R} \backslash \mathscr{C}$ and $p^{\prime \prime}(\bar{v})=0$ on $\mathscr{S}_{\boldsymbol{R}} \cap \mathscr{C}$. The intersection $\mathscr{S}_{L} \cap \mathscr{S}_{R}$ of the sonic loci consists of the inflection locus $\mathscr{I}$ and the

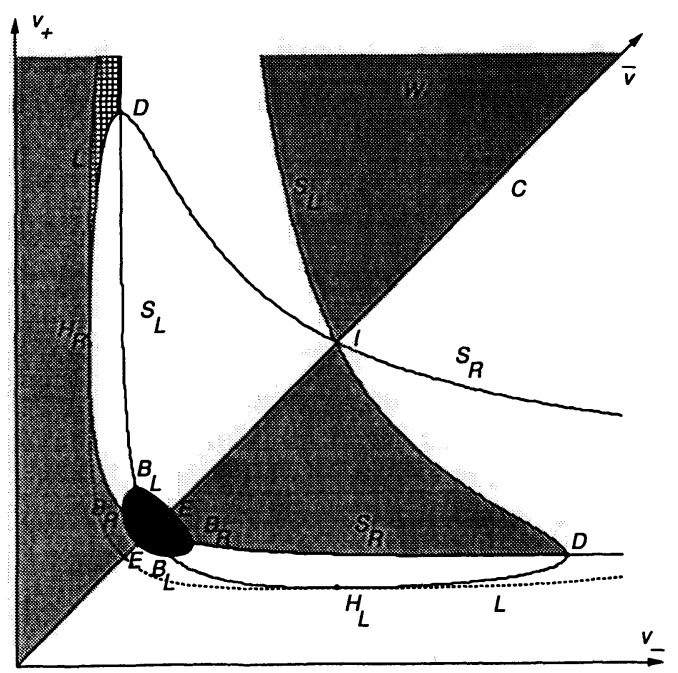

Fig. 7.2. The upper sheet $s=+\Psi\left(v_{-}, v_{+}\right)^{1 / 2}$ of $\mathscr{W}_{\text {base }}$ for a van der Walls gas. (Compare Figs. 2.2 and 7.2, noting that they are scaled differently.) Shown here are the characteristic manifold $\mathscr{C}$, the sonic loci $\mathscr{S}_{L}$ and $\mathscr{S}_{R}$, the inflection locus $\mathscr{I}$, the double sonic locus $\mathscr{D}$, the bifurcation loci $\mathscr{B}_{L}$ and $\mathscr{B}_{R}$, and the hysteresis loci $\mathscr{H}_{L}$ and $\mathscr{H}_{R}$. Shock waves in the shaded regions are limits of traveling-wave solutions of the system $u_{t}+p(v)_{x}=v u_{x x}, v_{t}-u_{x}=0$ as $v \rightarrow 0$; those in the cross-hatched region satisfy the Lax criterion but do not admit viscous profiles 
double sonic locus $\mathscr{D}$, which correspond to the maximum and the saddle points in Fig. 2.2, respectively. The inflection locus arises from the inflection point of the graph of $p$, while a double sonic point is obtained from a chord that is tangent at both its ends. The bifurcation loci $\mathscr{B}_{L}$ and $\mathscr{B}_{R}$ are subsets of $\mathscr{S}_{L}$ and $\mathscr{S}_{R}$, as indicated.

2. For (nondegenerate) quadratic models with $n=2$, the sonic loci are ruled surfaces within $\mathscr{W}$ when certain global coordinates $R, \kappa$, and $\Omega$ are used, the rules obtained by fixing $\Omega$. Relative to the characteristic manifold $R=0$, a sonic locus has two, one, or zero twists according as the projected coincidence locus is a hyperbola, a parabola, or an ellipse. Each hysteresis locus is the intersection of a ruled surface with a sonic locus. The double sonic locus comprises the rules at angles $\Omega$ that are roots of a homogeneous quadratic polynomial in $\cos \Omega$ and $\sin \Omega$.

3. For KKIT models, points on the right sonic locus within $\mathscr{W}_{\text {ruled }}$ correspond to sonic shock waves for the associated scalar conservation law. All points on $\mathscr{W}_{\text {contact }}$ are sonic.

As we now describe, the sonic loci have a geometric interpretation as the singularity loci for certain projection maps of $\mathscr{W}$.

Definition 7.17. Let $\tau_{-}: \mathscr{W} \rightarrow \mathscr{U} \times \mathbf{R}$ denote the map $(\bar{U}, R, \Omega, s) \mapsto\left(U_{-}, s\right)$ from $\mathscr{P} *$ to $\mathscr{U} \times \mathbf{R}$, as restricted to $\mathscr{W} \subset \mathscr{P} *$. Similarly, define $\tau_{+}: \mathscr{W} \rightarrow \mathscr{U} \times \mathbf{R}$ as the map $(\bar{U}, R, \Omega, s) \mapsto\left(U_{+}, s\right)$.

Theorem 7.18. The map $\tau_{-}: \mathscr{W} \rightarrow \mathscr{U} \times \mathbf{R}$ is singular precisely on $\mathscr{S}_{\mathbf{R}}$. Moreover, $p \in \mathscr{S}_{\boldsymbol{R}}$ is a fold point for $\tau_{-}$if and only if $p \notin \mathscr{H}_{\boldsymbol{R}}$.

Proof. Suppose that a vector $(\dot{\bar{U}}, \dot{R}, \dot{\Omega}, \dot{s}) \neq 0$ tangent to $\mathscr{W}$ at $p$ is mapped to zero by the derivative $\tau^{\prime}{ }_{-}$of $\tau_{-}$. Then $\dot{\bar{U}}=\frac{1}{2} r(\Omega) \dot{R}+\frac{1}{2} R r^{\prime}(\Omega) \dot{\Omega}$ and $\dot{s}=0$; in particular, $(\dot{R}, \dot{\Omega}) \neq 0$. Also, the condition for tangency of this vector to $\mathscr{W}$ simplifies to

$$
\left[\mathscr{F}_{R}+\frac{1}{2} \mathscr{F}_{\bar{U}} r(\Omega)\right] \dot{R}+\left[\mathscr{F}_{\Omega}+\frac{1}{2} R \mathscr{F}_{\bar{U}} r^{\prime}(\Omega)\right] \dot{\Omega}=0 .
$$

Thus $p \in \mathscr{S}_{\boldsymbol{R}}$. Conversely, if $p \in \mathscr{S}_{R}$, then there is a nonzero vector $(\dot{R}, \dot{\Omega})$ satisfying Eq. (7.12). Therefore with $\dot{\bar{U}}:=\frac{1}{2} r(\Omega) \dot{R}+\frac{1}{2} R r^{\prime}(\Omega) \dot{\Omega}$, the vector $(\dot{\bar{U}}, \dot{R}, \dot{\Omega}, 0) \neq 0$ is tangent to $\mathscr{W}$ and is mapped to zero by $\tau_{-}^{\prime}$, so that $\tau_{-}$is singular at $p$.

Consider the map $\psi: \mathscr{P}^{*} \rightarrow \mathscr{U} \times \mathbf{R} \times \mathbf{R}^{n}$ that carries the point $(\bar{U}, R, \Omega, s)$ to $\left(U_{-}, s, \mathscr{F}(\bar{U}, R, \Omega, s)\right)$. Because $\mathscr{W}$ is the submanifold of $\mathscr{P}^{*}$ where $\mathscr{F}=0, p$ is a fold point of $\tau_{-}$if and only if it is one for $\psi$; this follows from Lemma 3.6 using an argument similar to that given in the proof of Theorem 3.7. If $l$ and $r:=(\dot{R}, \dot{\Omega})$ are nonzero left and right null vectors of $B_{+}(\bar{U}, R, \Omega, s)$, respectively, then $\tilde{l}=\left(-l \mathscr{F}_{\bar{U}},-l \mathscr{F}_{s}, l\right)$ and $\tilde{r}=(\dot{\bar{U}}, \dot{R}, \dot{\Omega}, 0)$ are nonzero left and right null vectors of $\psi^{\prime}(p)$, where $\dot{\bar{U}}:=\frac{1}{2} r(\Omega) \dot{R}+\frac{1}{2} R r^{\prime}(\Omega) \dot{\Omega}$. Furthermore, as a simple calculation shows, $\tilde{l} \psi^{\prime \prime}(p) \tilde{r}^{2}=l \dot{B}_{+} r$, where $\dot{B}_{+}$denoted $B_{+}^{\prime}$ applied to $\tilde{r}$. Thus $p$ is a fold point for $\psi$ if and only if $p \notin \mathscr{H}_{\boldsymbol{R}}$.

It is natural to ask how many zeros $U$ the Rankine-Hugoniot equation (6.1) has for each $U_{0}$ and $s$. A detailed answer is given for systems of conservation laws with two equations in the work of Malta and Tomei [41]. The question can be rephrased within the present formalism: how many times does a shock curve with $U_{-}=U_{0}$ intersect a constant-speed slice of $\mathscr{W}$ corresponding to $s ?$ 
The lapproach of Malta and Tomei is to determine how the number of intersections $n\left(U_{0}, s\right)$ varies with $U_{0}$ and $s$. Notice first that $n\left(U_{0}, s\right)$ is constant in a neighborhood of any point $\left(U_{0}, s\right)$ that is a regular value of the projection map $\tau_{-}: \mathscr{W} \rightarrow \mathscr{U} \times \mathbf{R}$. Furthermore, because the singular locus of $\tau_{-}$is the sonic manifold $\mathscr{S}_{R}$, the locus $\mathscr{L}$ where $n\left(U_{0}, s\right)$ changes comprises points of $\mathscr{W}$ that project onto $\tau_{-}\left[\mathscr{S}_{R}\right]$. In other words, $\mathscr{L}$ consists of points $\left(U_{0}, U, s\right) \in \mathscr{W}$ such that the shock curve with $U_{-}=U_{0}$ contains a right sonic point $\left(U_{0}, \tilde{U}, s\right) \in \mathscr{S}_{R}$ with the same speed $s$. clearly, $\mathscr{L}$ contains $\mathscr{S}_{R}$; moreover, it is easy to verify that $\mathscr{L}$ contains points $\left(U, U_{0}, s\right) \in \mathscr{W}$ for which the shock curve with $U_{+}=U_{0}$ contains a left sonic point $\left(\tilde{U}, U_{0}, s\right) \in \mathscr{S}_{L}$, so that $\mathscr{L}$ contains $\mathscr{S}_{L}$.

The locus $\mathscr{L}$ is important for studying traveling-wave solutions of the parabolic equations (7.1). If $\mathscr{W}$ is regarded as parameterizing a family of dynamical systems, then $\mathscr{L}$ is the set of dynamical systems such that one of the critical points is not hyperbolic. For this reason we refer to $\mathscr{L}$ as the local bifurcation locus. Portions of $\mathscr{L}$ form the shock wave admissibility boundary.

\section{Examples.}

0 . For the scalar conservation law considered in Fig. 7.1, the boundary between the shaded and cross-hatched regions is part of $\mathscr{L}$. This boundary separates shock waves that satisfy the Oleinik condition from those that satisfy only the Lax condition. The other parts of $\mathscr{L} \backslash\left(\mathscr{S}_{L} \cup \mathscr{S}_{R}\right)$, which play no role in shock wave admissibility, have been omitted.

1. In gas dynamics, $\mathscr{L} \backslash\left(\mathscr{S}_{L} \cup \mathscr{S}_{R}\right)$ is represented in Fig. 7.2 by a dashed curve and by the boundary between the shaded and cross-hatched regions. The latter boundary separates physical from unphysical shock waves.

2. The local bifurcation locus for quadratic models is the subject of current research $[6,5]$.

3. For KKIT models, $\mathscr{L} \cap \mathscr{W}_{\text {ruled }}$ derives from the local bifurcation locus for the associated scalar conservation law.

\section{The Composite Foliation}

General scale-invariant solutions are composed of wave groups, each a sequence of adjoining rarefaction and shock waves. For a shock wave to appear next to a rarefaction wave, with no intervening constant sector, the shock wave must be sonic. Specifically, a rarefaction wave situated on the left of a left sonic shock wave is termed a left composite wave. Such a solution takes the form

$$
U(x, t)=\left\{\begin{array}{lll}
U_{0} & \text { if } \quad x<s_{0} t \\
\tilde{U}(x / t) & \text { if } \quad s_{0} t \leqq x<s t, \\
U_{+} & \text {if } \quad x>s t
\end{array}\right.
$$

here $\tilde{U}$ is a rarefaction satisfying Eq. (2.3), $U_{0}=\tilde{U}\left(s_{0}\right)$, and with the definition $U_{-}:=\tilde{U}(s)$, the triple $\left(U_{-}, U_{+}, s\right)$ satisfies the Rankine-Hugoniot condition Eq. (1.3). Similarly a rarefaction wave situated on the right of a right sonic shock wave is a right composite wave. 
Just as rarefaction and shock waves are organized into curves, the family of (left) composite waves with fixed $U_{0}$ forms a composite curve. Notice that the state $U_{0}$ uniquely determines the rarefaction function $\tilde{U}$; this is because $\widetilde{U}$ solves the ordinary differential equation (4.1) with initial condition $U_{0}$. Therefore a composite curve is, in essence, a family of sonic shock waves $\left(U_{-}, U_{+}, s\right)$ with $U_{-}=\tilde{U}(s)$ traversing a (projected) rarefaction curve in $\mathscr{U}$. Represented within the wave manifold $\mathscr{W}$, a composite curve lies in the left sonic locus $\mathscr{S}_{L}$, and its tangent projects, under the $U_{-}$map, to an eigenvector of $F^{\prime}\left(U_{-}\right)$corresponding to $s$.

To be more precise, consider a point $p=(\bar{U}, R, \Omega, s) \in \mathscr{Y}_{L}$, and let $U_{-}:=$ $\bar{U}-\frac{1}{2} \operatorname{Rr}(\Omega)$ be its $U_{-}$projection. Then the matrix $-s+F^{\prime}\left(U_{-}\right)$is singular; this follows from Eq. (7.5) when $p \notin \mathscr{C}$ and from Eq. (3.1) when $p \in \mathscr{C}$. Denote by $\mathscr{T}_{p}$ the set of vectors $(\bar{U}, \dot{R}, \dot{\Omega}, \dot{s})$ tangent to $\mathscr{S}_{L}$ at $p$ for which the $U_{-}$projection $\dot{U}_{-}:=\dot{\bar{U}}-\frac{1}{2} r(\Omega) \dot{R}-\frac{1}{2} R r^{\prime}(\Omega) \dot{\Omega}$ satisfies

$$
\left[-s+F^{\prime}\left(U_{-}\right)\right] \dot{U}_{-}=0 .
$$

This subspace is one-dimensional unless the matrix $-s+F^{\prime}\left(U_{-}\right)$, as restricted to $T_{p} \mathscr{S}_{L}$, has rank less than $n-1$. Away from such a singularity, Eq. (8.2) defines a smooth line field $\mathscr{T}$ on $\mathscr{S}_{L}$.

Definition 8.1. The (possibly singular) line field $\mathscr{T}$ on $\mathscr{S}_{\mathrm{L}}$ is called the composite line field. A composite curve is an integral curve of this line field. A similar definition applies to $\mathscr{S}_{R}$.
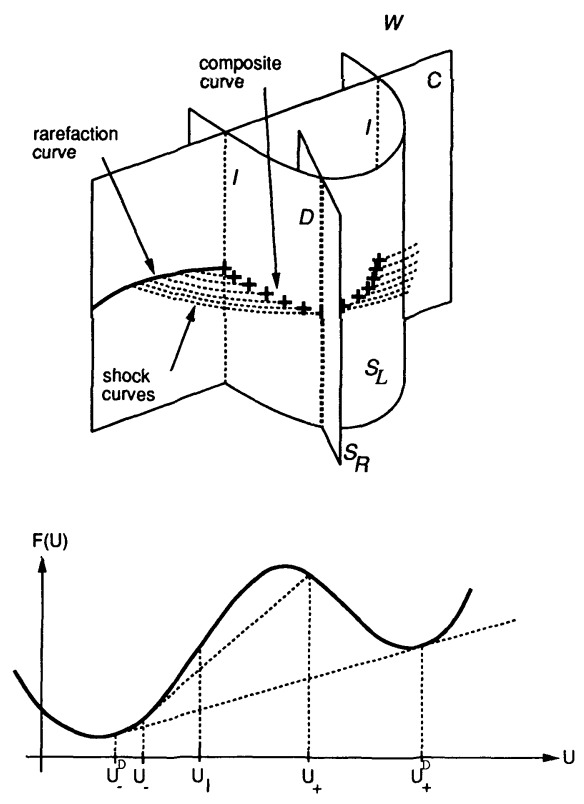

Fig. 8.1. A schematic depiction of a composite curve. A shock curve with fixed $U_{-}$is drawn from each point along a rarefaction curve in $\mathscr{C}$; the points where the shock curves intersect $\mathscr{S}_{L}$ form a composite curve. The flux diagram shown below indicates the analogous construction for a scalar conservational law $U_{t}+F(U)_{x}=0$ 
In other words, when $p \in \mathscr{S}_{L} \backslash \mathscr{C}$, a vector $\left(\dot{U}_{-}, \dot{U}_{+}, \dot{s}\right)$ belongs to the composite line field provided that it is tangent to $\mathscr{S}_{L}$ and $\left[-s+F^{\prime}\left(U_{-}\right)\right] \dot{U}_{-}=0$. If, on the other hand, $p \in \mathscr{S}_{L} \cap \mathscr{C}$, then a vector $(\dot{\bar{U}}, \dot{R}, \dot{\Omega}, \dot{s})$ projects to $\dot{U}_{-}:=\dot{\bar{U}}-\frac{1}{2} r(\Omega) \dot{R}$; therefore this vector belongs to the composite line field provided that it is tangent to $\mathscr{S}_{L}$ and $\left[-s+F^{\prime}(\bar{U})\right] \dot{\bar{U}}=0$. The construction of a composite curve is illustrated in Fig. 8.1.

There is an alternative perspective on the composite line field, based on drawing $U_{+}$-fixed shock curves. At a point $p \in \mathscr{S}_{L}$ there is, by definition, a nonzero vector tangent to $\mathscr{W}$ that belongs to both the shock line field $d U_{+}=0$ and the tangent hyperplane $d s=0$. The $U_{-}$projection $u_{-}$of such a vector serves to define the composite line field, as follows.

Proposition 8.2. At a point $p \in \mathscr{S}_{L}$, consider a nonzero vector tangent to $\mathscr{W}$ that belongs to both the shock line field $d U_{+}=0$ and the tangent hyperplane $d s=0$; let $u_{-}$ denote the $U_{-}$projection of this vector. Then

$$
\left[-s+F^{\prime}\left(U_{-}\right)\right] u_{-}=0 .
$$

Furthermore, $u_{-}$vanishes only if $p$ is an umbilic point.

Proof. If $p:=\left(U_{-}, U_{+}, s\right) \in \mathscr{S}_{L} \backslash \mathscr{C}$, we can use $U_{-}, U_{+}$, and $s$ as coordinates. Thus there is a vector $\left(u_{-}, u_{+}, \sigma\right) \neq 0$ tangent to $\mathscr{W}$ such that $u_{+}=0$ and $\sigma=0$; in particular, $u_{-} \neq 0$. Tangency to $\mathscr{W}$ requires $u_{-}$to satisfy Eq. (8.3).

If $p:=(\bar{U}, 0, \Omega, s) \in \mathscr{S}_{L} \cap C$, there is a vector $(\bar{u}, \rho, \omega, \sigma) \neq 0$ tangent to $\mathscr{W}$ such that $u_{+}:=\bar{u}+\frac{1}{2} \rho r(\Omega)=0$ and $\sigma=0 ; \bar{u}$ and $\omega$ satisfy $\mathscr{F}_{\bar{u}} \bar{u}+\mathscr{F}_{\Omega} \omega=0$. In other words there is a pair $(\rho, \omega) \neq 0$ such that

$$
-\frac{1}{2} \rho \mathscr{F}_{\bar{U}} r(\Omega)+\mathscr{F}_{\Omega} \omega=0 .
$$

Corresponding to the vector $(\bar{u}, \rho, \omega, \sigma)$ is its $U_{-}$projection, $u_{-}:=\bar{u}-\frac{1}{2} \rho r(\Omega)=$ $-\rho r(\Omega)$. Consequently, Eq. (8.3) holds.

If $u_{-}$vanishes, then according to Eq. (8.4), there is an $\omega \neq 0$ such that $\mathscr{F}_{\Omega} \omega=0$. Since $\mathscr{F}_{\Omega}=\left[-s+F^{\prime}(\bar{U})\right] r^{\prime}(\Omega)$, we see that $s$ is an eigenvalue of $F^{\prime}(\bar{U})$ corresponding to two independent eigenvectors, $r(\Omega)$ and $r^{\prime}(\Omega) \omega$. This implies that $p$ is an umbilic point.

Proposition 8.2 facilitates the computation of the composite line field. To determine $\mathscr{T}_{p}$ at the point $p \in \mathscr{S}_{L}$, first draw the fixed- $U_{+}$shock curve through $p$. Next let $u_{-}$be the $U_{-}$projection of a nonzero vector that is tangent to this curve; notice that $u_{-} \neq 0$ as long as $p$ is not an umbilic point. Finally, $\mathscr{T}_{p}$ consists of vectors tangent to $\mathscr{S}_{L}$ for which the $U_{-}$projection $\dot{U}_{-}$is parallel to $u_{-}$. (An example of this procedure is given below.)

The composite line field has a singularity at a point $p \in \mathscr{W}$ if and only if the subspace $\mathscr{T}_{p}$ has dimension greater than 1 . The following theorem determines these singularities (cf. App. B of ref. [44]).

Proposition 8.3. A point $p \in \mathscr{S}_{L} \backslash \mathscr{C}$ is a singularity of the (left) composite foliation if and only if $p \in \mathscr{D} \cap \mathscr{H}_{L}$ or $p \in \mathscr{S}_{L} \cap \mathscr{B}_{R}$. A point $p \in \mathscr{S}_{L} \cap \mathscr{C}$ is a singularity if and only if $p$ is an umbilic point, $p \in \mathscr{H}_{0}$, or $p \in \mathscr{B}_{0}$.

Proof. First suppose that $p \in \mathscr{S}_{L} \backslash \mathscr{C}$, so that the coordinates $\left(U_{-}, U_{+}, s\right)$ are valid in a neighborhood of $p$. In this neighborhood, the sonic surface $\mathscr{S}_{L}$ is defined by the 
equations $\mathscr{H}=0$ and $\operatorname{det}\left[-s+F^{\prime}\left(U_{-}\right)\right]=0$. One consequence of Assumption 7.5 is that $-s+F^{\prime}\left(U_{-}\right)$has rank $n-1$; therefore we can find nonzero left and right null vectors $l$ and $r$ of $-s+F^{\prime}\left(U_{-}\right)$, respectively, such that $l r \neq 0$.

By virtue of Eq. (8.2), a vector $\left(\dot{U}_{-}, \dot{U}_{+}, \dot{s}\right)$ in the line $\mathscr{T}_{p}$ has $\dot{U}_{-}=\dot{\alpha} r$ for some $\dot{\alpha} \in \mathbf{R}$. As a result, the conditions for tangency to $\mathscr{S}_{L}$ are

$$
-\left[U_{+}-U_{-}\right] \dot{s}+\left[-s+F^{\prime}\left(U_{+}\right)\right] \dot{U}_{+}=0
$$

and $-\dot{s} l r+l F^{\prime \prime}\left(U_{-}\right) \cdot\left(r \otimes \dot{U}_{-}\right)=0$ (see Lemma 5.5). Combining these conditions gives

$$
-\dot{\alpha}\left[U_{+}-U_{-}\right] \frac{l F^{\prime \prime}\left(U_{-}\right) r^{2}}{l r}+\left[-s+F^{\prime}\left(U_{+}\right)\right] \dot{U}_{+}=0 .
$$

Singularities of the composite line field occur where this linear system for $\dot{\alpha}$ and $\dot{U}_{+}$is rank deficient. Equivalently, singularities occur when there is a nonzero left null vector $\tilde{l} \neq 0$ of $-s+F^{\prime}\left(U_{+}\right)$and either $\tilde{l}\left[U_{+}-U_{-}\right]=0$ or $l F^{\prime \prime}\left(U_{-}\right) r^{2}=0$, i.e., $p \in \mathscr{B}_{R}$ or $p \in \mathscr{S}_{R} \cap \mathscr{H}_{L}$.

Now suppose that $p \in \mathscr{S}_{L} \cap \mathscr{C}$. If $p$ is an umbilic point, then $-s+F^{\prime}(\bar{U})$ has rank less than $n-1$, so that $p$ is a singularity of the composite line field. In the remainder of the proof, therefore, we can assume that $p$ is not an umbilic point, i.e., $r(\Omega)$ spans the null space of $-s+F^{\prime}(\bar{U})$.

Consider a vector $(\dot{\bar{U}}, \dot{R}, \dot{\Omega}, \dot{s})$ belonging to $\mathscr{T}_{p}$. Equation (8.2) implies that there is an $\dot{\alpha} \in \mathbf{R}$ such that $\dot{\bar{U}}=\frac{1}{2} \dot{\alpha} r(\Omega)$. To be tangent to $\mathscr{S}_{L}$, the vector must satisfy

$$
\frac{1}{2} \dot{\alpha} \mathscr{F}_{\bar{U}} r(\Omega)+\mathscr{F}_{\Omega} \dot{\Omega}+\mathscr{F}_{s} \dot{s}=0
$$

along with the equation $l \dot{B}_{-} r=0$, where $l$ and $r$ nonzero left and right null vectors of $B_{-}$, respectively, and $\dot{B}_{-}$denotes $B_{-}^{\prime}$ applied to the vector. If $p \in \mathscr{B}_{0}$, then Eq. (8.7) has rank less than $n$, so that there are fewer than $n+1$ linear conditions on $\dot{\alpha}$, $\dot{R}, \dot{\Omega}$, and $\dot{s}$; thus $p$ is a singularity for the composite line field. Therefore we can assume that $p \notin \mathscr{B}_{0}$ during the rest of the proof.

Comparing Eq. (8.7) to Eq. (8.4), we find that $\dot{s}=0$. Furthermore, the vector $r:=(-\dot{\alpha}, \dot{\Omega}) \neq 0$ is a right null vector of the matrix $\left(-\frac{1}{2} \mathscr{F}_{\bar{U}} r(\Omega), \mathscr{F}_{\Omega}\right)$, which is $B_{-}$ evaluated at $R=0$. A straightforward calculation shows that

$$
\dot{\alpha} l \dot{B}_{-} r=\left(\dot{\alpha}-\frac{1}{3} \dot{R}\right) l \dot{B}_{0} r
$$

where $\dot{B}_{0}$ denotes $B_{0}^{\prime}$ applied to $\left(\frac{1}{2} \dot{\alpha} r(\Omega), \dot{\Omega}, 0\right)$. Noting that $\dot{\alpha} \neq 0$ by Proposition 8.2 , the equation $l \dot{B}_{-} r=0$ represents the required single constraint on $\dot{\alpha}$ and $\dot{R}$ if and only if $l \dot{B}_{0} r \neq 0$, i.e., $p \notin \mathscr{H}_{0}$.

Examples.

1. For gas dynamics, let $\left(\dot{v}_{-}, \dot{v}_{+}, \dot{s}, \dot{\bar{u}}\right)$ denote a vector tangent to a fixed- $U_{+}$shock curve through a point of $\mathscr{S}_{L}$. Since $\dot{u}_{+}=0, \dot{v}_{+}=0$, and $\dot{s}=0$, the relationship $u_{+}=u_{-}-s\left(v_{+}-v_{-}\right)$on $\mathscr{W}$ implies that $\dot{u}_{-}=-s \dot{v}_{-} ;$this is the condition (8.3) on $\left(\dot{u}_{-}, \dot{v}_{-}\right)$. Now let $\left(\dot{v}_{-}, \dot{v}_{+}, \dot{s}, \dot{\bar{u}}\right)$ be tangent to $\mathscr{S}_{L}$. The conditions for tangency are obtained by differentiating the equations $s^{2}=\Psi\left(v_{-}, v_{+}\right)$and $\partial \Psi / \partial v_{-}=0$. Hence this vector belongs to the composite line field if and only if $\dot{u}_{-}=-s \dot{v}_{-}$. There are no singularities of the composite foliation for a van der Waals gas with $T \neq 1$.

2. The structure of the composite foliation for quadratic models remains to be determined. 
3. For KKIT models, composite curves arise from those for the associated scalar conservation law.

\section{Conclusions}

To conclude we discuss how the fundamental wave manifold provides a natural setting in which to study two fundamental problems on the theory of conservation lows: the physical admissibility of shock waves and the bifurcation of wave curves.

One of the most important issues in the theory of nonlinear conservation laws is choosing physically relevant discontinuous solutions. Not all solutions of the Rankine-Hugoniot conditions represent physical shock waves because dissipative effects have been neglected in the conservation laws. Thus the conservation laws must be supplemented by rules that mimic the neglected physics, called admissibility conditions. Mathematically, such rules are needed to avoid multiple solutions.

At present, the most encompassing criterion is the viscous profile criterion $[15,8,7]$ : a shock wave is admissible provided that it is the zero-viscosity limit of a traveling wave solution for a parabolic system such as system (7.1). Such a traveling wave corresponds to an orbit connecting the critical points of a dynamical system derived from the parabolic equations. The question of shock wave admissibility is tied, then, to how connecting orbits form or break as the shock wave is varied. Because bifurcation theory for dynamical systems in the plane is well understood $[47,59]$, the theory is developed especially well in the case $n=2$. For instance, Schecter and Shearer $[50,51,56]$ have obtained some detailed results concerning such bifurcations.

An important reason for introducing the fundamental wave manifold is that it parameterizes the dynamical systems associated to shock waves. In particular, bifurcations of the dynamical systems, which affect the connecting orbits, occur on certain submanifolds of $\mathscr{W}$. In fact, a point on a sonic locus $\mathscr{S}_{L}$ or $\mathscr{S}_{R}$, or more generally the local bifurcation locus $\mathscr{L}$, corresponds to a dynamical system that is unstable because one of its critical points is not hyperbolic. Other submanifolds of $\mathscr{W}$ correspond to global bifurcations, such as heteroclinic and homoclinic bifurcations, and are the subject of current research $[6,5]$. Thus the set of admissible shock wave can be pictured as an $(n+1)$-dimensional region within $\mathscr{W}$. We expect that this global perspective, based on the wave manifold, will be fruitful in the study of physically admissible shock waves.

The wave manifold is also natural for describing wave curve bifurcation. This phenomenon underlies how solutions of Riemann problems depend on the Riemann initial data, $U_{L}$ and $U_{R}$. In fact, the Riemann problems are solved by successively traversing wave curves; therefore abrupt changes in the nature of solutions are caused by the bifurcation of wave curves as the curve origin varies. Since wave curves are composed of segments of rarefaction, shock, and composite curves, wave curve bifurcation can, in turn, be reduced to the bifurcation of wave segments.

Wave curve bifurcation was studied systematically by F. Furtado [14]; this theoretical development draws on the experience of several authors who studied model systems of conservation laws [55, 54, 24, 28, 29, 21, 25]. Furtado identified the complete list of loci where wave curves bifurcate. However, the list is along and complicated, and the bifurcation loci do not have an intuitive interpretation. 
Furthermore, the list is derived assuming the Lax admissibility criterion; an extension to other criteria is straightforward but tedious.

The global perspective of the wave manifold clarifies Furtado's result: wave curve bifurcation is simply loss of transversality between the wave foliations (rarefaction, shock and composite) and the characteristic manifold $\mathscr{C}$ or the local bifurcation locus $\mathscr{L}$. More generally, $\mathscr{C}$ and $\mathscr{L}$ can be regarded as the boundaries of the regions of Lax admissibility; therefore wave curve bifurcation with respect to other admissibility criteria is loss of transversality between wave foliations and the admissibility boundary. There are three causes for loss of transversality: (a) the foliation is singular; (b) the admissibility boundary is singular (e.g., at corners); or (c) a curve in the foliation is tangent to the admissibility boundary.

In the present work we have shown how these bifurcation loci arise. Concerning singularities of the foliations, we established that: the shock foliations are singular on the secondary bifurcation loci $\mathscr{B}_{L}$ and $\mathscr{B}_{R}$; the rarefaction foliation is singular on the singularity locus $\mathscr{B}_{0}$; and the composite foliations are singular at umbilic points, singularities of $\mathscr{I}$, and on the loci $\left(\mathscr{D} \cap \mathscr{H}_{L}\right) \cup\left(\mathscr{S}_{L} \cap \mathscr{B}_{R}\right)$ and $\left(\mathscr{D} \cap \mathscr{H}_{R}\right) \cup$ $\left(\mathscr{S}_{R} \cap \mathscr{B}_{L}\right)$. Under certain nondegeneracy conditions, each of these bifurcation loci has codimension 2 relative to the manifold foliated by the curves (viz., $\mathscr{W}, \mathscr{C}$, and $\mathscr{S}_{L}$ or $\mathscr{S}_{R}$, respectively).

The boundary of the Lax admissibility region is formed by portions of $\mathscr{C}$ and $\mathscr{L}$; the local bifurcation locus $\mathscr{L}$ is composed of the sonic loci $\mathscr{S}_{L}$ and $\mathscr{S}_{R}$ and their "extensions." Assuming that these loci are smooth manifolds, the admissibility boundary has singularities only at the intersections of these manifolds, where the boundary has corners. (See Figs. 7.1 and 7.2.) Thus singularities of the admissibility boundary occur on $\mathscr{I}$ and $\mathscr{D}$ and certain "extended loci," all of which have codimension 2 in $\mathscr{W}$. Finally, we have shown that: the shock foliation is tangent to the sonic loci on the hysteresis loci $\mathscr{H}_{L}$ and $\mathscr{H}_{R}$; and the rarefaction foliation is tangent to the sonic loci (in fact, to $\mathscr{I}$ ) on the exceptional inflection locus $\mathscr{H}_{0}$.

While work remains to prove that the list just sketched is complete, we conjecture that loss of transversality gives rise to all of Furtado's bifurcation loci. Thus the bifurcation of wave curves, as well as physical admissibility of shock waves, is clarified by a global approach based on the fundamental wave manifold.

Acknowledgements. We thank Sunčica Čanić, Frederico Furtado, James Glimm, and Qiu-Ping $\mathrm{Xu}$ for stimulating discussions, and Sunčica Čanic for help with the figures. The hospitality of the Institute for Mathematics and its Applications, the Department of Applied Mathematics and Statistics at the State University of New York at Stony Brook, and the Instituto de Matemática Pura e Aplicada is gratefully acknowledged.

\section{Appendix. General Systems of Conservation Laws}

In this Appendix we give an intrinsic definition of the fundamental wave manifold $\mathscr{W}$ for general systems of conservation laws with one spatial variable. For such systems, the state space $\mathscr{U}$ is a smooth $n$-dimensional manifold, and the conserved quantity $U(x, t) \in \mathscr{U}$ is governed by the partial differential equations.

$$
H(U)_{t}+F(U)_{x}=0 .
$$

The flux functions $H$ and $F$ are smooth maps from $\mathscr{U}$ to $\mathbf{R}^{n}$. 
Suppose that a weak solution of Eq. (A.1) has a jump discontinuity along a smooth curve in the $(t, x)$-plane. This discontinuity is specified by the inclination angle $\theta$ and by the limiting states $U_{+}$and $U_{-}$; by convention, $\left(n_{t}, n_{x}\right)=(\cos \theta, \sin \theta)$ is normal to the discontinuity curve, and $U_{+}$lies on the side to which the normal points. The angle and states conform to the Rankine-Hugoniot condition

$$
\cos \theta\left[H\left(U_{+}\right)-H\left(U_{-}\right)\right]+\sin \theta\left[F\left(U_{+}\right)-F\left(U_{-}\right)\right]=0 .
$$

A characteristic direction for system (A.1) at a state $\bar{U}$ corresponds to an inclination angle and a line $r$ tangent to $\mathscr{U}$ at $\bar{U}$ such that

$$
\left[\cos \theta H^{\prime}(\bar{U})+\sin \theta F^{\prime}(\bar{U})\right] r=0 .
$$

We make the basic assumption on system (A.1) that each characteristic and each discontinuity has a unique inclination angle. More precisely: we assume that (A) if $H\left(U_{+}\right)=H\left(U_{-}\right)$and $F\left(U_{+}\right)=F\left(U_{-}\right)$, then $U_{+}=U_{-}$; and that (B) if $r \in T_{\bar{U}} \mathscr{U}$ is such that $H^{\prime}(\bar{U}) r=0$ and $F^{\prime}(\bar{U}) r=0$, then $r=0[61$, p. 116]. These assumptions reflect the physical requirement that the flux functions distinguish points of $\mathscr{U}$.

The quantities entering the Rankine-Hugoniot condition are points $\left(U_{-}, U_{+}, \theta\right)$ in $\mathscr{U} \times \mathscr{U} \times S^{1}$, but the two points $\left(U_{+}, U_{-}, \theta+\pi\right)$ and $\left(U_{-}, U_{+}, \theta\right)$ represent the same discontinuity. Denote by $\mathscr{P}$ the ambient space obtained from $\mathscr{U} \times \mathscr{U} \times S^{1}$ by identifying such pairs of points. We are interested in the subset of $\mathscr{P}$ defined by the Rankine-Hugoniot condition. However, Eq. (A.2) is singular when $U_{+}=U_{-}=: \bar{U}$ and $\theta$ is such that Eq. (A.3) holds for some $r$. This singularity will be removed by blowing up $\mathscr{P}$ over the diagonal submanifold $\Delta$ where $U_{+}=U_{-}$.

The blow-up procedure derives from the classical quadratic transformation of algebraic geometry. This construction extends to schemes [20], complex spaces [11], and vector bundles [3]. Our application requires blowing up a smooth manifold over a submanifold; we define this procedure in several steps.

First let $V$ be a vector space, and let $P V$ denote the projective space of lines through the origin in $V$. The blow-up of $V$ over 0 [3, p. 268, 20, p. 28] is the set $\Gamma_{0} V:=\{(x, l) \in V \times P V: x \in l\}$, i.e., the disjoint union of the lines in $V$. The blow-up has a standard structure as an analytic manifold; indeed, it is the total space of the universal line bundle $\Gamma_{0} V \rightarrow P V$. The projection $\pi: \Gamma_{0} V \rightarrow V$ of the first factor is called the collapsing map, and the set $0^{*}:=\pi^{-1}[0] \cong P V$ is a submanifold of codimension 1 called the exceptional hypersurface.

The standard atlas $\left\{\left(\mathcal{O}_{i}, \varphi_{i}\right): i=1, \ldots, \operatorname{dim} V\right\}$ for $\Gamma_{0} V$ is defined as follows. For $y \in V$ with $y \neq 0$, let $[y]:=\{\alpha y: \alpha \in \mathbf{R}\} \in P V$ denote the line through $y$. Then we define the set $\mathcal{O}_{i}$ as $\left\{(x,[y]): y \in V, x \in[y], y_{i} \neq 0\right\}$ and the map $\varphi_{i}: \mathcal{O}_{i} \rightarrow V$ as taking $(x,[y])$ to $\left(y_{1} / y_{i}, \ldots, x_{i}, \ldots, y_{n} / y_{i}\right)$. Clearly $\Gamma_{0} V$ is covered by the $\mathcal{O}_{i}$, each $\varphi_{i}$ is a bijection, and the transition maps $\varphi_{i}{ }^{\circ} \varphi_{j}^{-1}$ are analytic on $\mathcal{O}_{i} \cap \mathcal{O}_{j}$. To make a connection with the construction of the blow-up in Sect. 2, consider any norm on $V$, such defines a sphere $S V \subset V$. Then each point $(R, \Omega) \in \mathbf{R} \times S V$ maps to a point $(R \Omega,[\Omega]) \in \Gamma_{0} V$. By identifying antipodal points $(R, \Omega)$ and $(-R,-\Omega)$ within $\mathbf{R} \times S V$ we obtain a space diffeomorphic to $\Gamma_{0} V$. As a set, $\Gamma_{0} V$ can be identified with $(V \backslash\{0\}) \cup P V$, where the isomorphism carries $(x, l)$ to $x$ if $x \neq 0$ and to $l$ otherwise.

Next suppose that $\mathcal{O}$ is an open subset of $V$ with $0 \in \mathcal{O}$. Then the blow-up of $\mathcal{O}$ over 0 is $\Gamma_{0} \mathcal{O}:=\Gamma_{0} V \cap(\mathcal{O} \times P V)$. More generally, suppose that $\mathcal{O}_{i} \subseteq V_{i}$ for $i=1,2$ with $0 \in \mathcal{O}_{2}$, and let $M=\mathcal{O}_{1} \times \mathcal{O}_{2}$ and $S=\mathcal{O}_{1} \times\{0\}$. Then the blow-up of $M$ over the 
submanifold $S$ is $\Gamma_{S} M=\mathcal{O}_{1} \times \Gamma_{0} \mathcal{O}_{2}$, and the collapsing map $\pi: \Gamma_{S} M \rightarrow M$ is defined to carry $\left(x_{1}, x_{2}, l_{2}\right)$ to $\left(x_{1}, x_{2}\right)$. The blow-up can be identified with the set $(M \backslash S) \cup\left(S \times P V_{2}\right)$, in which case the collapsing map is the identity map on $M \backslash S$ and the projection of the first factor on $S \times P V_{2}$. Notice that $S \times V_{2} \cong T M / T S$ is the normal bundle $N S$ of $S$ as a submanifold of $M$, so that $S \times P V_{2}$ is the projective normal bundle $P N S$. In essence, $S$ is blown up to be the hypersurface $S^{*}:=\pi^{-1}[S] \cong$ $P N S$ within $\Gamma_{S} M$.

Extending this definition to general manifolds requires understanding the effect of a change of coordinates. Consider a diffeomorphism $\psi$ between $\widetilde{M}=\widetilde{\mathcal{O}}_{1} \times \widetilde{\mathcal{O}}_{2}$ and $M=\mathcal{O}_{1} \times \mathcal{O}_{2}$ that restricts to a diffeomorphism between the submanifolds $\tilde{S}=$ $\tilde{\mathcal{O}}_{1} \times\{0\}$ and $S=\mathcal{O}_{1} \times\{0\}$. Then we define the blow-up of $\psi$, denoted $\Gamma \psi: \Gamma_{\tilde{S}} M \rightarrow$ $\Gamma_{S} M$, as follows. Writing $\psi=\left(\psi_{1}, \psi_{2}\right)$, let

$$
\Psi_{2}\left(x_{1}, x_{2}\right):=\int_{0}^{1} \frac{\partial \psi_{2}}{\partial x_{2}}\left(x_{1}, \alpha x_{2}\right) d \alpha .
$$

Notice that $\Psi_{2}\left(x_{1}, x_{2}\right) x_{2}=\psi_{2}\left(x_{1}, x_{2}\right)$ and that $\Psi_{2}\left(x_{1}, 0\right)=\left(\partial \psi_{2} / \partial x_{2}\right)\left(x_{1}, 0\right)$ is nonsingular. If $x_{2} \in l_{2}$, therefore, $\Psi_{2}\left(x_{1}, x_{2}\right) l_{2}$ is a line and $\psi_{2}\left(x_{1}, x_{2}\right)$ belongs to it, as seen by considering the cases $x_{2} \neq 0$ and $x_{2}=0$. Hence we define $\Gamma \psi\left(x_{1}, x_{2}, l_{2}\right):=$ $\left(\psi_{1}\left(x_{1}, x_{2}\right), \psi_{2}\left(x_{1}, x_{2}\right), \Psi_{2}\left(x_{1}, x_{2}\right) l_{2}\right)$. That this map is a diffeomorphism is easily verified. The blow-up of $\psi$ reduces to $\psi$ on $\tilde{M} \backslash \tilde{S}^{*}$, while on $\tilde{S}^{*}$ it carries $\left(x_{1}, 0, l_{2}\right)$ to $\left(\psi_{1}\left(x_{1}, 0\right), 0,\left(\partial \psi_{2} / \partial x_{2}\right)\left(x_{1}, 0\right) l_{2}\right)$. The latter map is the induced map PN $\psi: P N \widetilde{S} \rightarrow$ $P N S$ between projective normal bundles.

A related construction gives rise to a universal mapping property for blow-ups. Suppose that $\tilde{S}^{*}=\tilde{\mathcal{O}}_{1} \times\{0\}$ is a hypersurface in $\tilde{\Gamma}=\tilde{\mathcal{O}}_{1} \times \tilde{\mathcal{O}}_{2}$ (i.e., $0 \in \tilde{\mathcal{O}}_{2} \subseteq \mathbf{R}$ ), and that $\tilde{\pi}: \tilde{\Gamma} \rightarrow M$ is a smooth map such that (a) $\tilde{S}^{*}=\tilde{\pi}^{-1}[S]$ and (b) the induced map $N \tilde{\pi}: N \tilde{S}^{*} \rightarrow N S$ is injective. Then there is a unique smooth map $\rho: \tilde{\Gamma} \rightarrow \Gamma_{S} M$ such that $\tilde{\pi}=\pi \circ \rho$. Indeed: when $\left(x_{1}, x_{2}\right) \notin \tilde{S}^{*}, \rho\left(x_{1}, x_{2}\right)$ must equal $\left(\tilde{\pi}_{1}\left(x_{1}, x_{2}\right), \tilde{\pi}_{2}\left(x_{1}, x_{2}\right)\right.$, $\left[\tilde{\Pi}_{2}\left(x_{1}, x_{2}\right)\right]$ ), where $\tilde{\Pi}_{2}$ is defined by the formula analogous to Eq. (A.4); and assumption (b), which means that the vector $\left(\partial \tilde{\pi}_{2} / \partial x_{2}\right)\left(x_{1}, 0\right) \neq 0$ for all $\left(x_{1}, 0\right) \in \tilde{S}^{*}$, implies that this definition of $\rho$ extends smoothly to $\tilde{S}^{*}$.

Finally, suppose that $M$ is a smooth manifold and that $S$ is a submanifold. Let $P N S \rightarrow S$ be the projective normal bundle of $S$ as a submanifold of $M$, which has fiber $P\left(T_{x} M / T_{x} S\right)$ above $x \in S$. Define the blow-up of $M$ over $S$ to be the set $\Gamma_{S} M:=(M \backslash S) \cup P N S$ and endow it with the following atlas of charts. If $p \in M \backslash S$, then any chart $(\mathcal{O}, \varphi)$ for $M$ with $p \in \mathcal{O} \subseteq M / S$ serves as a chart for $\Gamma_{S} M$. If, on the other hand, $p \in P N S_{x}$ for some $x \in S$, then consider a chart $(\mathcal{O}, \varphi)$ for $M$ at $x$; assume that this chart conforms to the submanifold $S$ of $M$, in the sense that $\varphi[\mathcal{O}]=\mathcal{O}_{1} \times \mathcal{O}_{2} \subseteq V_{1} \times V_{2}$ and $\varphi[\mathcal{O} \cap S]=\mathcal{O}_{1} \times\{0\}$. Such a chart induces an isomorphism between the projective normal bundle $\left.P N S\right|_{\mathcal{O}}$ over $\mathcal{O}$ and $\mathcal{O}_{1} \times P V_{2}$, and therefore between the subset $\left.(\mathcal{O} \backslash S) \cup P N S\right|_{\mathcal{O}}$ and $\Gamma_{\mathcal{O}_{1} \times\{0\}} \mathcal{O}_{1} \times \mathcal{O}_{2}=\mathcal{O}_{1} \times \Gamma_{0} \mathcal{O}_{2}$. Under this isomorphism, we obtain charts for $\Gamma_{S} M$ from charts for $\mathcal{O}_{1} \times \Gamma_{0} \mathcal{O}_{2}$. (For instance, we may use coordinates $\left(x_{1}, R, \Omega\right) \in \mathcal{O}_{1} \times \mathbf{R} \times S V_{2}$.) The resulting atlas makes $\Gamma_{\mathrm{S}} M$ a smooth manifold because the overlap maps are the blow-ups of diffeomorphisms like those discussed above.

The collapsing map $\pi: \Gamma_{S} M \rightarrow M$ is defined to be the identity map on $M \backslash S$ and the bundle projection on PNS; it is smooth because it reduces to the collapsing map on each chart. The preimage $S^{*}:=\pi^{-1}[S]=P N S$ is a submanifold of codimension 1 , 
called the exceptional hypersurface, since it is the exceptional hypersurface in each chart. Finally, a universal mapping property characterizing the blow-up follows from the local version described above.

Proposition A.1. Suppose $\tilde{\pi}: \tilde{\Gamma} \rightarrow M$ is a smooth map such that (a) $\tilde{S}^{*}:=\tilde{\pi}^{-1}[S]$ is a hypersurface of $\tilde{\Gamma}$ and (b) the induced map $N \tilde{\pi}: N \widetilde{S}^{*} \rightarrow N S$ between normal bundles is injective. Then there exists a unique smooth map $\rho: \tilde{\Gamma} \rightarrow \Gamma_{S} M$ such that $\tilde{\pi}=\pi \circ \rho$.

In our application to conservation laws we let $\mathscr{P} *:=\Gamma_{\Delta} \mathscr{P}$ be the blow-up of $\mathscr{P}$ over the diagonal submanifold $\Delta$. Composing the left-hand side of Eq. (A.2) with the collapsing map yields a smooth map $\mathscr{H} \circ \pi: \mathscr{P}^{*} \rightarrow \mathbf{R}^{n}$. The zero-set of $\mathscr{H} \circ \pi$ is the union of two parts: the exceptional hypersurface $\mathscr{P}_{0}^{*}$ and the fundamental wave set $\mathscr{W}$ (see Fig. 2.3). Indeed, just as in Sect. 2, $\mathscr{H} \circ \pi$ exhibits an explicit factor of $R$ when expressed in local coordinates about the exceptional hypersurface; the zero-set of the remaining factor is defined to be $\mathscr{W}$. Under mild regularity assumptions, $\mathscr{W}$ is a submanifold of $\mathscr{P}^{*}$ that is transverse to $\mathscr{P}_{0}^{*}$, so that $\mathscr{C}:=\mathscr{W} \cap \mathscr{P}_{0}^{*}$ is a submanifold of $\mathscr{W}$. Our basic assumption on system (A.1) implies that the inclination angle $\theta$ can be expressed as a function of coordinates for $\mathscr{W}$.

It was shown in Sect. 6 that the rarefaction line field on $\mathscr{C}$ is induced from the shock line field on $\mathscr{W}$ by the projection that sets the $R$-component of the tangent vector to zero. In the present setting, this projection $p:\left.\left.T \Gamma_{S} M\right|_{S} \rightarrow T \Gamma_{S} M\right|_{S}$ is defined as follows. By localizing to a chart, we reduce the problem to defining a projection $p: T_{(0,[y])} \Gamma_{0} V \rightarrow T_{(0,[y])} \Gamma_{0} V$, where $y \in V$ with $y \neq 0$. Corresponding to the standard charts $\left(\mathcal{O}_{i}, \varphi_{i}\right), i=1, \ldots, \operatorname{dim} V$, for $\Gamma_{0} V$, there are bijections $\sigma_{i}: T_{(0,[y])} \Gamma_{0} V \simeq V$ such that if $(0,[y]) \in \mathcal{O}_{i} \cap \mathcal{O}_{j}$, then $\sigma_{i}^{\circ} \sigma_{j}^{-1}$ is the Jacobian $\left(\varphi_{i}^{\circ} \varphi_{j}^{-1}\right)^{\prime}\left(\varphi_{j}(0,[y])\right)$. Let $p_{i}: V \rightarrow V$ be the projection that sets the $i^{\text {th }}$ component of $\dot{y} \in V$ to zero. It is easily verified that $\left(\sigma_{i}^{\circ} \sigma_{j}^{-1}\right) \circ p_{j}$ coincides with $p_{i}^{\circ}\left(\sigma_{i}^{\circ} \sigma_{j}^{-1}\right)$. Therefore there is a unique projection $p: T_{(0,[y])} \Gamma_{0} V \rightarrow T_{(0,[y])} \Gamma_{0} V$ such that $\sigma_{i} \circ p^{\circ} \sigma_{i}^{-1}=p_{i}$ when $(0,[y]) \in \mathcal{O}_{i}$. This definition globalizes to give a smooth projection $p:\left.\left.T \Gamma_{S} M\right|_{S} \rightarrow T \Gamma_{S} M\right|_{S}$. In terms of the local coordinates $\left(x_{1}, R, \Omega\right) \in \mathcal{O}_{1} \times \mathbf{R} \times S V_{2}$ for $\Gamma_{2} M, p$ takes $\left(\dot{x}_{1}, \dot{R}, \dot{\Omega}\right) \in V_{1} \times \mathbf{R} \times T S V_{2}$ to $\left(\dot{x}_{1}, 0, \dot{\Omega}\right)$.

The universal mapping property gives a global definition for the coordinates used throughout the main body of the paper. To see this, let $M$ be the space $\mathbf{R}^{n} \times \mathbf{R}^{n} \times S^{1}$ modulo the identification of each point $(-x,-y, \theta+\pi)$ with its antipode $(x, y, \theta)$, and define $\mathscr{G}: \mathscr{P} \rightarrow M$ by

$$
\mathscr{G}\left(U_{-}, U_{+}, \theta\right)=\left(H\left(U_{+}\right)-H\left(U_{-}\right), F\left(U_{+}\right)-F\left(U_{-}\right), \theta\right) .
$$

Also, let $S \simeq \mathbf{R} P^{1}$ be the submanifold of points $(0,0, \theta) \in M$. Then $\mathscr{G}_{\circ} \pi: \mathscr{P}^{*} \rightarrow M$ satisfies hypotheses (a) and (b) of Prop. A.1; indeed, these hypotheses follow directly from assumptions (A) and (B), respectively. Therefore there is a unique map $\rho: \mathscr{P}^{*} \rightarrow \Gamma_{S} M$ such that $\mathscr{G}_{\circ} \pi=\rho^{\circ} \pi_{M}$, where $\pi_{M}: \Gamma_{S} M \rightarrow M$ is the collapsing map.

Since $M$ is $\mathbf{R}^{2 n} \times S^{1}$ with antipodal points identified, the blow-up $\Gamma_{S} M$ is $\mathbf{R} \times S^{2 n-1} \times S^{1}$ modulo identifying the points $\left(-R,-\Omega_{1},-\Omega_{2}, \theta\right)$ and $\left(-R, \Omega_{1}\right.$, $\left.\Omega_{2}, \theta+\pi\right)$ with $\left(R, \Omega_{1}, \Omega_{2}, \theta\right)$. (Here $\left(\Omega_{1}, \Omega_{2}\right) \in S^{2 n-1} \subset \mathbf{R}^{n} \times \mathbf{R}^{n}$.) The subset of $\Gamma_{S} M$ consisting of points such that $\cos \theta \Omega_{1}+\sin \theta \Omega_{2}=0$ forms an $(n+1)$-dimensional submanifold $\mathscr{R} \subset \Gamma_{M} S$ that is transverse to $\mathscr{P}_{0}^{*}$. On $\mathscr{R}$ we can write $\Omega_{1}=-\sin \theta \Omega$ and $\Omega_{2}=\cos \theta \Omega$ for some $\Omega \in S^{n-1}$, so that $\mathscr{R}$ has coordinates $(R, \Omega, \theta)$. Furthermore the map $\rho$ induces a map from $\mathscr{W}$ to $\mathscr{R}$, thereby defining $R$ and $\Omega$ globally throughout $\mathscr{W}$. 


\section{References}

1. Azevedo, A., Marchesin, D.: Multiple Viscous Profile Riemann Solutions in Mixed EllipticHyperbolic Models for Flow in Prorous Media. In: Hyperbolic Equations that Change Type, Keyfitz, B., Shearer, M. (eds). IMA Volumes in Mathematics and its Applications, vol. 27, pp. 1-17. Berlin, Heidelberg, New York: Springer 1990

2. Bethe, H.: The Theory of Shock Waves for an Arbitrary Equation of State. Clearinghouse for Federal Scientific and Technical Information. U.S. Dept. of Commerce, Rep. No. PB-32189, Washington, DC, 1942

3. Bott, R., Tu, L.: Differential forms in algeraic topology. Berlin, Heidelberg, New York: Springer 1982

4. Brio, M.: Admissibility conditions for Weak Solutions of Non-Strictly Hyperbolic Systems. In: Nonlinear Hyperbolic Equations - Theory, Computational Methods, and Applications, Ballmann, J., Jeltsch, R. (eds). Notes on Numerical Fluid Mechanics, vol. 24, 43-50. Braun-Schweig: Vieweg 1989

5. Čanić, S.: State Univ. of New York of Stony Brook. Ph.D. Thesis, in preparation, 1992

6. Čanić, S., Plohr, B.: A global approach to shock wave admissibility. State Univ. of New York at Stony Brook, Report No. SUNYSB-AMS-91-10, 1991

7. Conley, C., Smoller, J.: Viscosity matrices for two-dimensional nonlinear hyperbolic systems. Commun. Pure Appl. Math. 23, 867-884 (1970)

8. Courant, C., Friedrichs, K.: Supersonic flow and shock waves. New York, NY: John Wiley 1948

9. Dafermos, C.: Structure of solutions of the Riemann problem for hyperbolic systems of conservation laws. Arch. Rational Mech. Anal. 53, 203-217 (1974)

10. Dafermos, C., DiPerna, R.: The Riemann problem for certain classes of hyperbolic systems of conservation laws. J. Differ. Eqs. 20, 90-114 (1976)

11. Fischer, G.: Complex Analytic Geometry. Lect. Notes in Math., vol. 538, Berlin, Heidelberg, New York: Springer 1976

12. Foy, L.: Steady state solutions of hyperbolic systems of conservation laws with viscous terms. Commun. Pure Appl. Math. 17, 177-188 (1964)

13. Freistühler, H.: Instability of vanishing viscosity approximation to hyperbolic systems of conservation laws with rotational invariance. J. Differ. Eq. 87, 205-226 (1990)

14. Furtado, F.: Structural stability of nonlinear waves for conservation laws. New York Univ., Ph.D. Thesis, 1989

15. Gelfand, I.: Some problems in the theory of quasi-linear equations. Uspekhi Mat. Nauk 14, 87-158 (1959), Am. Math. Soc. Transl. 295-381 (1963)

16. Glimm, J.: Nonlinear and Stochastic Phenomena: The Grand Challenge for Partial Differential Equations. SIAM Review 33, 626-643 (1991)

17. Golubitsky, M., Guillemin, V.: Stable mappings and their singularities. Berlin, Heidelberg, New York: Springer 1973

18. Golubitsky, M. Schaeffer, D.: Singularities and groups in bifurcation theory. Berlin, Heidelberg, New York: Springer 1984

19. Gomes, M. E.: Riemann problems requiring a viscous profile entropy condition, Adv. Appl. Math. 10, 285-323 (1989)

20. Hartshorne, R.: Algebraic geometry, Berlin, Heidelberg, New York: Springer 1977

21. Holden, H.: On the Riemann problem for a prototype of a mixed type conservation law. Commun. Pure Appl. Math. 40, 229-264 (1987)

22. Isaacson, E.: Global Solution of a Riemann Problem for a Nonstrictly Hyperbolic System of Conservation Laws Arising in Enhanced Oil Recovery. Rockefeller Univ., preprint, 1981

23. Isaacson, E., Marchesin, D., Plohr, B.: Transitional waves for conservation laws. SIAM J. Math. Anal. 21, 837-866 (1990)

24. Isaacson, E., Marchesin, D., Plohr, B., Temple, J. B.: The Riemann problem near a hyperbolic singularity: The classification of quadratic Riemann problems I. SIAM J. Appl. Math. 48, 1009-1032 (1988)

25. - Multiphase flow models with singular Riemann problems. Mat. Appl. Comput. (1992) (to appear)

26. Isaacson, E., Temple, J. B.: Examples and classification of non-strictly hyperbolic systems of conservation laws. Proceedings of the Am. Math. Soc. Meeting, Anaheim, CA (1985) 
27. - Analysis of a singular hyperbolic system of conservation laws. J. Diff. Equations 65, 250-268 (1986)

28. - : The Riemann problem near a hyperbolic singularity II. SIAM J. Appl. Math. 48, 1287-1301 (1988)

29. $\longrightarrow$ : The Riemann problem near a hyperboiic singularity III. SIAM J. Appl. Math. 48, 1302-1312 (1988)

30. Kan, P.-T.: On the Cauchy Problem of a $2 \times 2$ System of Nonstrictly Hyperbolic Conservation Laws. New York Univ., Ph.D. Thesis, 1989

31. Keyfitz, B., Kranzer, H.: The Riemann problem for a class of hyperbolic conservation laws exhibiting a parabolic degeneracy. J. Diff. Equations 47, 35-65 (1983)

32. Kobayashi, S., Nomizu, K.: Foundations of differential geometry. Vol I, New York, NY: Interscience 1863

33. Lax, P.: Hyperbolic systems of conservation laws II. Commun. Pure Appl. Math. 10, 537-566 (1957)

34. - : Hyperbolic systems of conservation laws and the mathematical theory of shock waves. Regional Conference Series Lectures in Applied Mathematics, vol. 11, Society for Industrial and Applied Mathematics, Philadelphia, PA, 1973

35. Leibovich, L.: Solutions of the Riemann problem for hyperbolic systems of quasi-linear equations without convexity conditions. J. Diff. Equations 45, 81-90 (1974)

36. Liu, T.-P.: The Riemann problem for general $2 \times 2$ conservation laws. Trans. Am. Math. Soc. 199, 89-112 (1974)

37. 18, 218-234 (1975)

38. - Admissible solutions of hyperbolic conservation laws. Mem. Am. Math. Soc. 30, 603-634 (1981)

39. $\quad$ Nonlinear stability of shock waves for viscous conservation laws. Mem. Am. Math. Soc. 56, 1-108 (1985)

40. Liu, T.-P., Xin, X.: Overcompressive Shock Waves, in Nonlinear Evolution Equations that Change Type, Keyfitz, B., Shearer, M. (eds). IMA Volumes in Mathematics and its Applications, vol. 27, pp. 139-145. Berlin, Heidelberg, New York: Springer 1990

41. Malta, I., Tomei, C.: Singularities of vector fields arising from one-dimensional Riemann problems. J. Diff. Equations 94, 165-190 (1991)

42. Marchesin, D., Palmeira, C. F.: Topology of elementary waves of mixed systems of conservation laws. In preparation, 1992

43. Medeiros, H.: Stability of hyperbolic singularities for conservation laws in models of flow of three-phase fluids in porous media. Pontifícia Universidade Católica do Rio de Janeiro, Ph.D. Thesis (in Portuguese), 1989

44. Menikoff, R., Plohr, B.: The Riemann problem for fluid flow of real materials. Rev. Mod. Phys. 61, 75-130 (1989)

45. Oleinnik, O.: On the uniqueness of the generalized solution of the Cauchy problem for a non-linear system of equations occuring in mechanics. Uspekhi Mat. Nauk 12, 169-176(1957)

46. Palmeira, C. F.: Line fields defined by eigenspaces of derivatives of maps from the plane to itself. Proceedings of the IV ${ }^{\text {th }}$ International Conference of Differential Geometry, Santiago de Compostela, Spain (1988)

47. Peixoto, M.: Structural stability on two dimensional manifolds. Topology 1, 101-120 (1962)

48. Riemann, B.: Uber die Fortpflanzung ebener Luftwellen von endlicher Schwingungsweite. Göttingen Abhandlungen 8, 43 (1958)

49. Schaeffer, D., Shearer, M.: The classification of $2 \times 2$ systems of non-strictly hyperbolic conervation laws, with application to oil recovery. Commun. Pure Appl. Math. 40, 141-178 (1987)

50. Schecter, S.: Simultaneous equilibrium and heteroclinic bifurcation of planar vector fields via the Melnikov integral. Nonlinearity 3, 79-99 (1990)

51. Schecter, S., Shearer, M.: Undercompressive shocks for nonstrictly hyperbolic conservation laws. J. Dyn. Diff. Equations 3, 199-271 (1991)

52. Shearer, M.: Admissibility criteria for shock wave solutions of a system of conservation laws of mixed type. Proc. R. Soc. Edinburgh 93A, 233-244 (1983)

53. — : The Riemann problem for $2 \times 2$ systems of hyperbolic conservation laws with case I quadratic nonlinearities. J. Diff. Equations 80, 343-363 (1989) 
54. Shearer, M., Schaeffer, D.: Riemann problems for nonstrictly hyperbolic $2 \times 2$ systems of conservation laws. Trans. Am. Math. Soc. 304, 267-306 (1987)

55. Shearer, M., Schaeffer, D., Marchesin, D., Paes-Leme, P.: Solution of the Riemann problem for a protoype $2 \times 2$ system of non-strictly hyperbolic conservation laws. Arch. Rational Mech. Anal. 97, 299-320 (1987)

56. Shearer, M., Schecter, S.: Riemann Problems Involving Undercompressive Shocks. In: PDEs and Continuum Models of Phase Transitions, Rascle, M., Serre, D., Slemrod, M. (eds). Lect. Notes in Phys., vol. 344, pp. 187-200. Berlin, Heidelberg, New York: Springer 1989

57. Slemrod, M.: Admissibility criteria for propagating phase boundaries in a van der Waals fluid. Arch. Rational Mech. Anal. 81, 303-319 (1983)

58. Smoller, J., Johnson, J.: Global solutions for an extended class of hyperbolic systems of conservation laws. Arch. Rational Mech. Anal. 32, 169-189 (1969)

59. Stomayor, J.: Generic one-parameter families of vector fields on two-dimensional manifolds. Publ. Math. IHES 43, 5-46 (1974)

60. Wendroff, B.: The Riemann Problem for Materials with Non-Convex Equations of State: I Isentropic Flow: II General Flow, J. Math. Anal. and Appl. 38, 454-466; 640-648 (1972)

61. Whitham, G.: Linear and nonlinear waves, New York, NY: John Wiley 1974

62. Wu, C.: On MHD intermediate Shocks. Geophys. Res. Lett. 14 (668-671)

63. Xu, Q.-P.: State Univ. of New York at Stony Brook, Ph.D. Thesis, in preparation, 1992

Communicated by A. Jaffe 